\title{
Colored HOMFLY polynomials of knots presented as double fat diagrams
}

\author{
A. Mironov, ${ }^{a, b, c, d}$ A. Morozov, ${ }^{b, c, d}$ An. Morozov, ${ }^{b, c, d, e}$ P. Ramadevi ${ }^{f}$ and \\ Vivek Kumar Singh ${ }^{f}$ \\ ${ }^{a}$ Theory Department, Lebedev Physics Institute, \\ Leninsky prospekt, Moscow 119991, Russia \\ ${ }^{b}$ ITEP, \\ Bol. Cheremushkinskaya, Moscow 117218, Russia \\ ${ }^{c}$ National Research Nuclear University MEPhI, \\ Kashirskoe highway, Moscow 115409, Russia \\ ${ }^{d}$ Institute for Information Transmission Problems, \\ Bol. Karetny, Moscow 127994, Russia \\ ${ }^{e}$ Laboratory of Quantum Topology, Chelyabinsk State University, \\ Bratiev Kashirinykh st., Chelyabinsk 454001, Russia \\ ${ }^{f}$ Department of Physics, Indian Institute of Technology Bombay, \\ Powai, Mumbai 400076, India \\ E-mail: mironov@lpi.ru, morozov@itep.ru, morz_0@mail.ru, \\ ramadevi@phy.iitb.ac.in, vivek.bhu2009@gmail.com
}

ABSTRACT: Many knots and links in $S^{3}$ can be drawn as gluing of three manifolds with one or more four-punctured $S^{2}$ boundaries. We call these knot diagrams as double fat graphs whose invariants involve only the knowledge of the fusion and the braiding matrices of four-strand braids. Incorporating the properties of four-point conformal blocks in WZNW models, we conjecture colored HOMFLY polynomials for these double fat graphs where the color can be rectangular or non-rectangular representation. With the recent work of Gu-Jockers, the fusion matrices for the non-rectangular [21] representation, the first which involves multiplicity is known. We verify our conjecture by comparing with the [21] colored HOMFLY of many knots, obtained as closure of three braids. The conjectured form is computationally very effective leading to writing [21]-colored HOMFLY polynomials for many pretzel type knots and non-pretzel type knots. In particular, we find class of pretzel mutants which are distinguished and another class of mutants which cannot be distinguished by [21] representation. The difference between the [21]-colored HOMFLY of two mutants seems to have a general form, with $A$-dependence completely defined by the old conjecture due to Morton and Cromwell. In particular, we check it for an entire multi-parametric family of mutant knots evaluated using evolution method.

Keywords: Quantum Groups, Chern-Simons Theories, Topological Field Theories ARXIV EPRINT: 1504.00371 


\section{Contents}

1 Introduction 1

$\begin{array}{llr}\text { I } & \text { Diagrammar } & 7\end{array}$

$\begin{array}{lll}2 & \text { Chern-Simons evolution } & 7\end{array}$

3 Pretzel fingers and propagators $\quad 10$

4 Other building blocks $\quad 11$

4.1 Non-pretzel fingers 11

4.2 Horizontal loop 11

4.3 Horizontal braid 12

5 Some examples of double fat diagrams and knot polynomials 13

$\begin{array}{lll}5.1 & \text { Pretzel knots and links } & 14\end{array}$

$\begin{array}{lll}5.1 .1 & \text { Trefoil } & 14\end{array}$

5.1.2 Twist knots: thin, triple-boundary (pretzel) $(\overline{1}|\overline{1}| \overline{2 k-1}) \quad 15$

$\begin{array}{lll}5.1 .3 & \text { Odd antiparallel pretzel knots } & 15\end{array}$

$\begin{array}{lll}5.1 .4 & \text { Pure parallel pretzel knots } & 15\end{array}$

$\begin{array}{ll}\text { 5.1.5 Mixed parallel-antiparallel pretzel knots } & 15\end{array}$

$\begin{array}{lll}5.2 & \text { Non-pretzel fingers } & 16\end{array}$

$\begin{array}{lll}5.3 & \text { Double fat graph presentation of knots from three-strand braids } & 16\end{array}$

$\begin{array}{ll}5.3 .1 s=1: \text { the starfish case } & 17\end{array}$

5.3.2 Generic four-box 3-strand braids beyond starfish family 18

$\begin{array}{ll}5.4 \text { Diagrams with non-tadpole propagators } & 19\end{array}$

$\begin{array}{ll}\text { 5.4.1 The form for ppS block } & 19\end{array}$

$\begin{array}{lll}5.4 .2 & \text { Double braids } & 19\end{array}$

5.4.3 Pretzel fingers connected via horizontal braid 20

$\begin{array}{ll}\text { II Examples } & 21\end{array}$

6 Racah matrices $\quad 21$

6.1 Generalities 21

6.2 Racah matrices for few symmetric representations 23

6.3 Representation [21] 24

$\begin{array}{lll}7 & \sigma \text {-factors and other technicalities } & 29\end{array}$

$\begin{array}{ll}7.1 \text { Relevant choices } & 29\end{array}$

$\begin{array}{lll}7.1 .1 & 3 \text { fingers } & 29\end{array}$ 
$\begin{array}{lll}\text { 7.1.2 } & \text { More fingers } & 30\end{array}$

$\begin{array}{lll}7.2 & \text { Sign dependence on knots } & 30\end{array}$

7.3 Explicit calculations in the pretzel case 31

7.3.1 Parallel 31

$\begin{array}{lll}\text { 7.3.2 } & \text { Even antiparallel } & 32\end{array}$

$\begin{array}{ll}\text { 7.3.3 Odd antiparallel } & 32\end{array}$

7.4 Checks 33

7.4.1 Special polynomials 34

7.4.2 Alexander polynomials for 1-hook representations 34

$\begin{array}{lll}7.4 .3 & \text { Jones polynomials } & 34\end{array}$

7.4.4 The weak form of differential expansion 34

7.4.5 Quasiclassical expansion and Vassiliev invariants 35

8 Mutants $\quad 35$

8.1 Generalities 35

8.2 Examples of mutants 36

8.3 HOMFLY for the Pretzel mutants 36

$\begin{array}{lll}8.4 & \text { KTC mutants } & 37\end{array}$

$\begin{array}{ll}8.5 & \text { Other mutant pairs }\end{array}$

9 Evolution method in application to mutant families $\quad 40$

$\begin{array}{lll}9.1 & \text { Twist knots [40, 41, 78] } & 40\end{array}$

9.2 4-finger pretzel knots 41

10 Conclusion $\quad 42$

A Examples of [21]-colored HOMFLY 43

A.1 A list of knots with up to 10 intersections 43

$\begin{array}{lll}\text { A.2 Some thin knots } & 50\end{array}$

A.3 The first thick knots 51

A.3.1 3-strand cases $\quad 52$

A.3.2 4-parallel pretzel finger cases $\quad 54$

A.3.3 Realizations from [43] $\quad 54$

$\begin{array}{lll}\text { A.4 Mutant pairs } & 59\end{array}$

\section{Introduction}

$R$-colored HOMFLY polynomial [1-8] for any knot $\mathcal{K}$ is defined as the Wilson loop average in Chern-Simons theory on three-manifold $\mathcal{M}[9]$

$$
H_{R}^{\mathcal{K} \subset \mathcal{M}} \sim \int\left(\operatorname{Tr}_{R} P \exp \oint_{\mathcal{K}} \mathbf{A}\right) \exp \left\{\frac{\kappa}{4 \pi} \int_{\mathcal{M}} \operatorname{Tr}_{a d j}\left(\mathbf{A} d \mathbf{A}+\frac{2}{3} \mathbf{A}^{3}\right)\right\} D \mathbf{A} .
$$


In fact, these polynomials are Laurent polynomials (that is, all the coefficients are integers) in two variables $q=\exp \left(\frac{2 \pi i}{\kappa+N}\right)$ and $A=q^{N}$ for knots in $\mathcal{M}=S^{3}$. Here $N$ denotes the rank of the $\mathrm{SU}(N)$ gauge group and $k$ is the Chern-Simons coupling.

These polynomials can be lifted to a positive integer superpolynomial [11]-[35] by introducing an additional parameter $\mathbf{t}$. The deviation from $\mathbf{t}=-1$ is known as $\beta$ deformation [36]. There is no combinatoric approach of obtaining superpolynomials for any knot. It is still a mystery to obtain superpolynomials for non-rectangular representations $R$ (i.e. when the corresponding Young diagram $R$ is not rectangular) [37-39]. Even the evaluation of colored HOMFLY polynomials for such representations is a difficult task.

Two recent achievements open a way to perform explicit calculation of the colored HOMFLY polynomials beyond rectangular representations.

- The first of them is the evaluation of the 3 -strand knot polynomials for representation $R=[21]$ in [37, 40] and the data of Racah matrices $S$ and $\bar{S}$ given in ref. [41].

- The second is the conjectured expression [42-47] for knots in $S^{3}$ obtained from gluing three-manifolds with one or more four-punctured $S^{2}$ boundaries. We call such diagrams as double fat tree diagrams (see definitions below). The unreduced colored HOMFLY polynomial involving the Racah matrices for such knot diagrams will be

$$
H_{R}^{\mathcal{K}}=\sum_{\left\{X_{\mu}, a_{i}^{(\mu)}\right\}} \sigma_{X \mid a} \prod_{\text {vertices } \mu} d_{X_{\mu}} \prod_{\text {propagators } \mu \nu} \frac{d_{R}}{\sqrt{d_{X_{\mu}} d_{X_{\nu}}}} \mathcal{A}_{X_{\mu} X_{\nu}}^{a^{(\mu)} a_{j}^{(\nu)}}
$$

We indicate each of the three-manifolds $\mu$ 's with $k$ four-punctured $S^{2}$ boundaries as a $k_{\mu}$-valent vertex. Further, we place representation $X_{\mu} \in R \otimes \bar{R}$ or $R \otimes R$, depending on the configuration and orientations of the knot diagram (see example). We will have additional indices $a_{i}^{(\mu)}=1, \ldots, m_{X_{\mu}}$ in the summation besides $X_{\mu}$ 's, whenever $X$ has a non-trivial multiplicity $m_{X}$. The weight factor is the product of quantum dimensions $d_{X}$ of $X$. Additional factors are just signs, $\sigma= \pm 1$, which appear only for non-trivial multiplicities. We will see in section 7 that these $\sigma$ 's are crucially important for non-rectangular $R$ including $R=[21]$. The form of $\mathcal{A}_{X_{\mu} X_{\nu}}^{a_{i}^{(\mu)} a_{j}^{(\nu)}}$ will depend on braiding and fusion matrices as explained in eq. (1.6).

\section{Definitions}

Tree diagram: we denote the knots drawn as double fat diagrams as ordinary trees with vertices $\mu$ 's of arbitrary valencies $k_{\mu}$ 's:

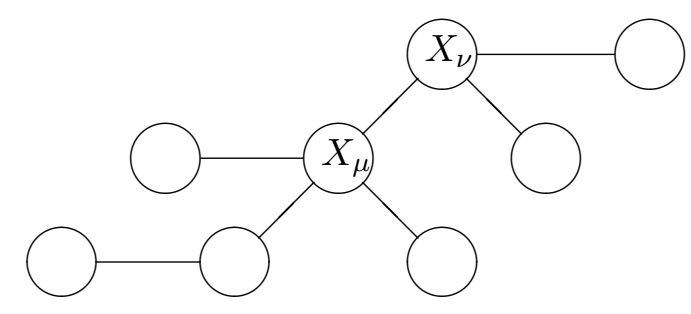




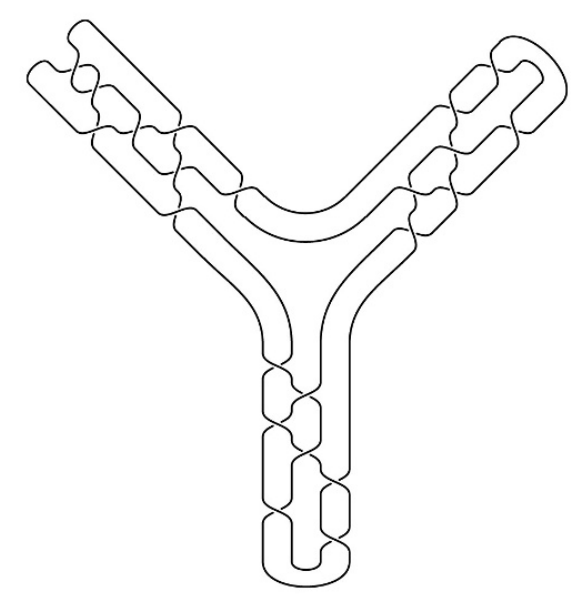

Figure 1. Knot drawn as double fat diagram.

An example. Suppose a knot can be presented in the form as in figure 1 (which we call double fat diagram).

The above double fat knot diagram is presentable as a single trivalent vertex connected by edges to three monovalent vertices. In this example, it is three four-strand braids (propagators, or edges) connected by double lines into the vertex $X$, we call it the double fat diagram. In the field theory context, this particular diagram has a peculiar shape of a starfish with three fingers. The colored HOMFLY polynomial of such knots will be

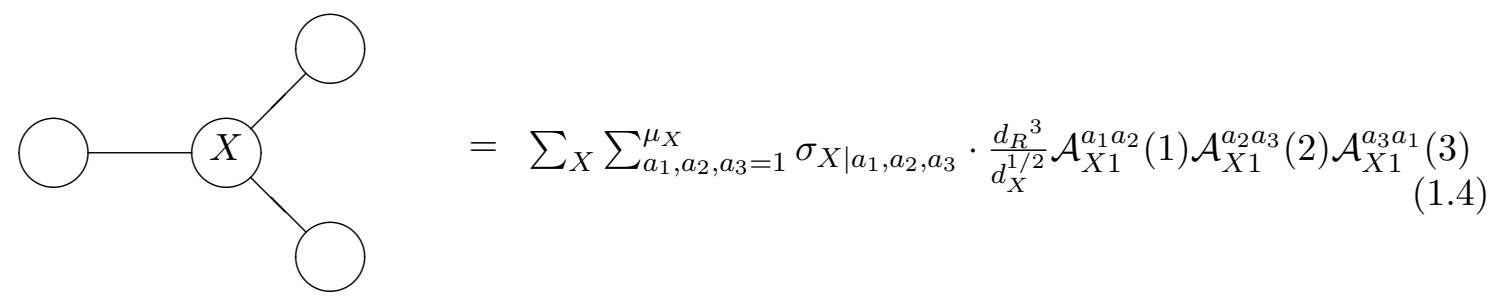

The subscript 1 in $\mathcal{A}_{X 1}^{a_{i} a_{j}}$ indicates the singlet representation (3.1). In this paper, we will call graphs involving a $k$-valent vertex connected to $k$ monovalent vertices as starfish diagram with $k$ fingers. 
Edge in the tree diagram: we always consider each edge in (1.3) being a 4-strand braid:
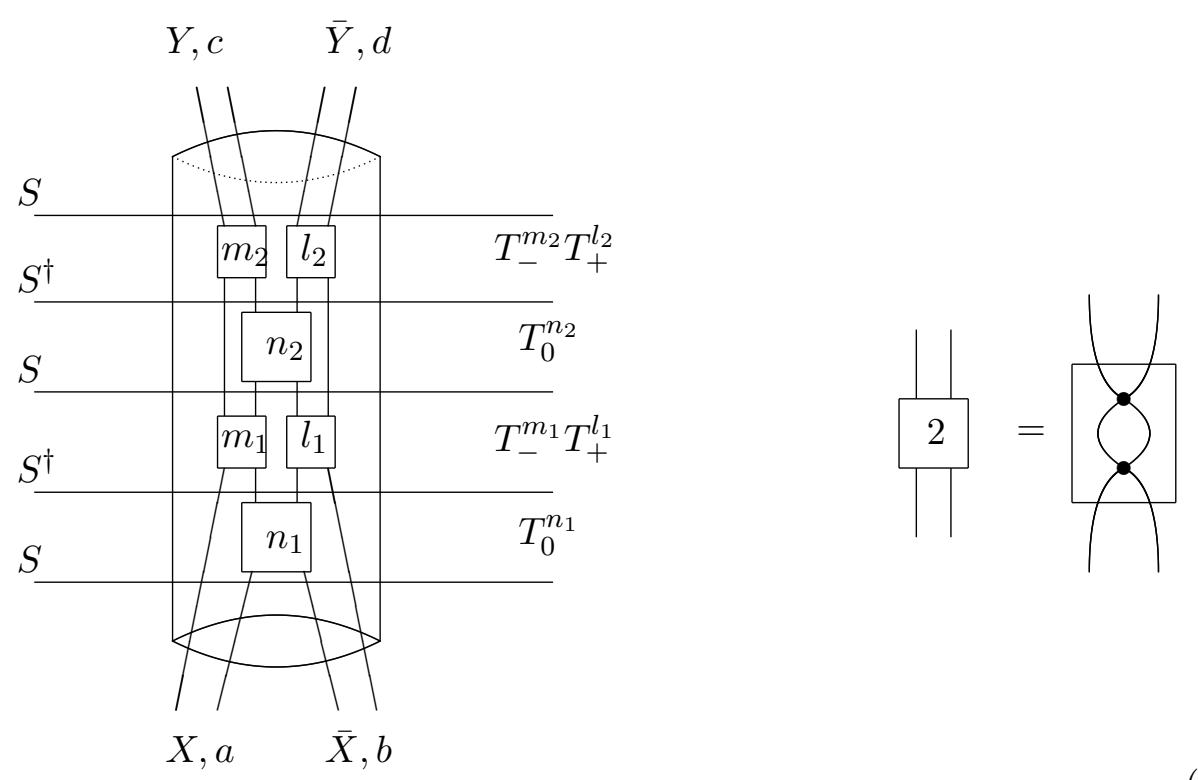

Boxes in this picture denote vertical 2-strand braids of given lengths, which can be parallel or antiparallel, depending on the directions of arrows (which in their turn depend on parity of the lengths). The number of boxes in each propagator can be arbitrary. For a given braid word in the above picture, we will have to insert the appropriate $S, S^{\dagger}$ fusion matrices and suitable powers of the braiding matrices $T_{-}, T_{+}, T_{0}$ as highlighted. These four-strand braids are called propagators which connects two different vertices. We put represention $X$ and $Y$ on the two vertices as explained below.

Vertex in tree diagram: each vertex $\mu$ in (1.3) is the cyclic junctions of $k_{\mu} 4$-strand braids:

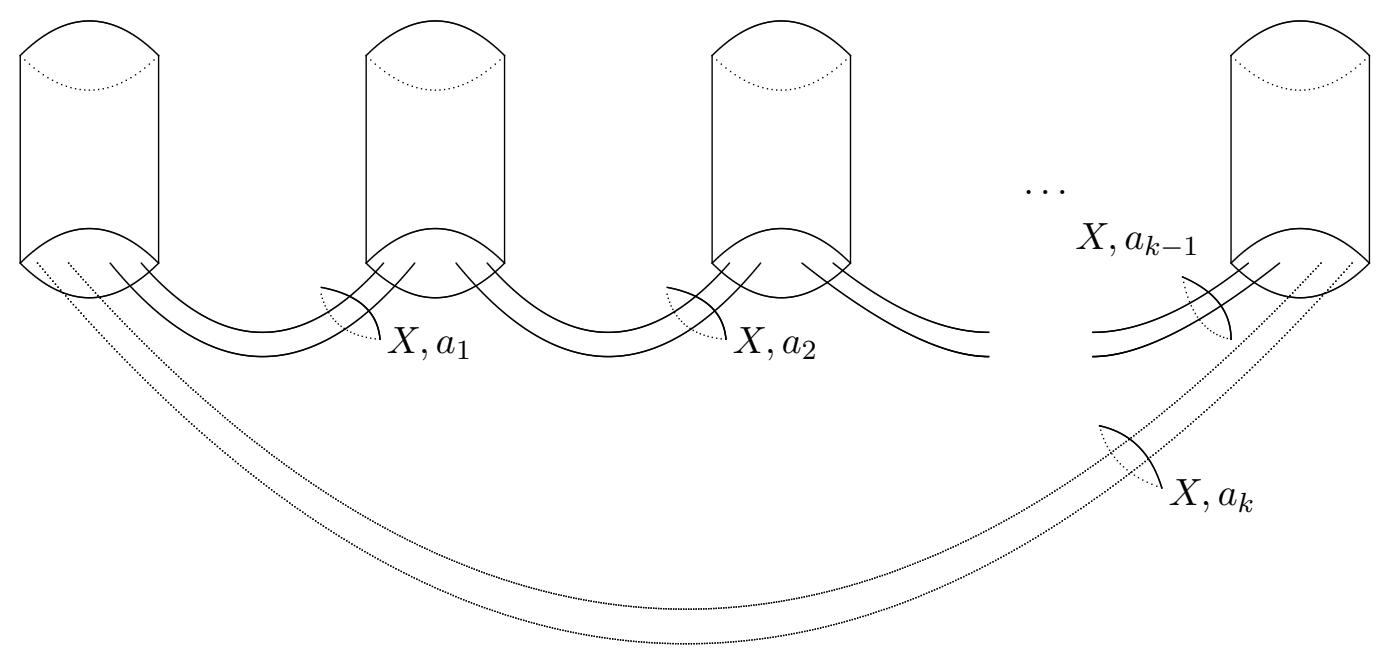


With each such vertex $\mu$ one associates a representation $X_{\mu}$. The crucial feature of this construction is the selection rule for propagators: the two representations $X, \bar{X}$ at one end of the four-strand braid (1.5) differ by conjugation, while the additional indices $a$ and $b$ (multiplicity indices) can be different.

Now we are in a position to write the explicit form for matrix element $\mathcal{A}_{Y c d, X a b}$, for every propagator connecting two vertices with index $X a b$ and $Y c d$. The form for the matrix, in terms of the fusion or Racah matrix $S$ and the braiding matrices $T_{0}, T_{-}, T_{+}$, will be

$$
\mathcal{A}=S \cdot \prod_{\alpha}^{\leftarrow}\left(T_{-}^{m_{\alpha}} T_{+}^{l_{\alpha}} S^{\dagger} T_{0}^{n_{\alpha}} S\right)
$$

Interchanging $m_{\alpha}$ and $l_{\alpha}$ at each level $\alpha$ inside every fingeris a mutation transform (provided one considers only the tree diagrams), and in fact one can perform a mutation to change $m_{\alpha}, l_{\alpha} \rightarrow m_{\alpha}+k_{\alpha}, l_{\alpha}-k_{\alpha}$ with any $k_{\alpha}$. For symmetric representations $R$, the HOMFLY polynomial depends only on the sums $m_{\alpha}+l_{\alpha}$ as there is no distinction between $T_{-}$and $T_{+}$. However, for non-rectangular $R$, the invariant depends on $m_{\alpha}$ and $l_{\alpha}$ separately leading to a possibility of distinguishing mutants.

In the case of the fundamental representation $R=[1]$ of $S U_{q}(2)$, i.e. for $N=2$, expressions (1.6) involve

$$
S=S^{\dagger}=\left(\begin{array}{cc}
\frac{1}{[2]} & \frac{\sqrt{[3]}}{[2]} \\
\frac{\sqrt{[3]}}{[2]} & -\frac{1}{[2]}
\end{array}\right), \quad T=\left(\begin{array}{cc}
1 & 0 \\
0 & -q^{2}
\end{array}\right)
$$

in vertical framing. We need to add frame corrections to $T$-matrix resulting in different form for parallel and antiparallel braiding. For higher $N$, there are two different Racah matrices, $S$ for parallel and $\bar{S}$ for antiparallel braids respectively and the respective frame corrected braiding matrices $T, \bar{T}$ as given below:

$$
\begin{array}{ll}
S=\frac{1}{[N]}\left(\begin{array}{cc}
\sqrt{\frac{[N][N-1]}{[2]}} & \sqrt{\frac{[N][N+1]}{[2]}} \\
\sqrt{\frac{[N][N+1]}{[2]}}-\sqrt{\frac{[N][N-1]}{[2]}}
\end{array}\right), & T=-\frac{1}{q A}\left(\begin{array}{cc}
1 & 0 \\
0 & -q^{2}
\end{array}\right) \\
1 & \sqrt{[N-1][N+1]} \\
\bar{S}=\frac{1}{[N]}\left(\begin{array}{cc}
{[N-1}
\end{array}\right), & \bar{T}=\left(\begin{array}{cc}
1 & 0 \\
0 & -A
\end{array}\right)
\end{array}
$$

where $A=q^{N}$. Two types of matrices $S$ remain sufficient for symmetric, rectangular and non-rectangular representations. However, for non-rectangular $R$ we will have additional indices $a$ and $b$ added to $X$ to incorporate the multiplicity of some representations.

For any representation $R$, the first row in the Racah matrices $S$ and $\bar{S}$ are always constructed from the quantum dimensions:

$$
\begin{aligned}
& S_{1 X}^{a b}=\frac{\sqrt{d_{X}}}{d_{R}} \delta^{a b}, \quad X \in R \otimes R, \quad 1 \in R \otimes \bar{R} \\
& \bar{S}_{1 X}^{a b}=\frac{\sqrt{d_{X}}}{d_{R}} \delta^{a b}, \quad X \in R \otimes \bar{R}
\end{aligned}
$$


This property is crucial for self-consistency of (1.2) when an edge is a tadpole. In fact, the role of the end-vertex in a tree diagram (with the sum over the corresponding $X$ ) is to imitate gluing of the caps like

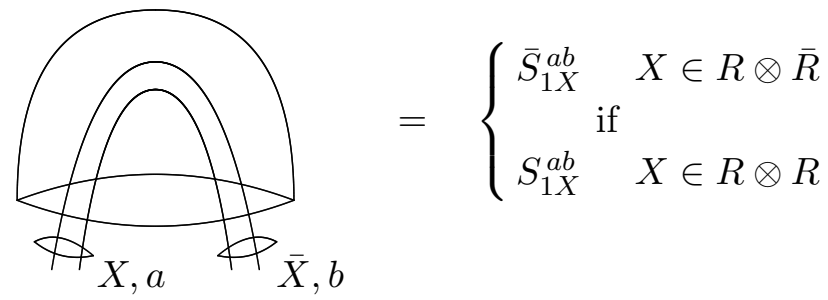

with a singlet representation $1 \in R \otimes \bar{R}$, or

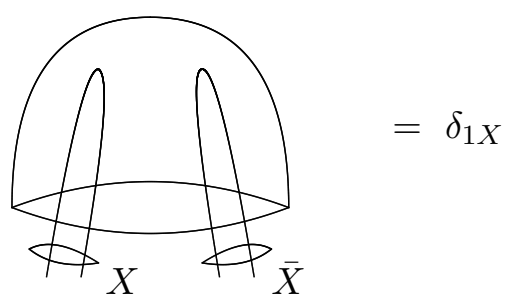

Relations (1.10) ensure that each tadpole (end-edge of a branch in the tree, which we call finger) is nothing but the matrix element $\mathcal{A}_{1 X}^{a b}$. Hence, we omit the cycles at the end-edges of the tree branches. Additional universally applicable simplifications emerge from the elementary $S, T$-matrix identities, like the celebrated $(S T)^{3}=1$ in the case of $R=[1]$ for $S U_{q}(2)$, and their generalization to arbitrary representations of $S U_{q}(N)$ (6.4).

The set of fat-tree diagrams is quite ample. According to [43, 44], which contains fat diagram presentations of knots upto 10 crossings in Rolfsen table [48] (with the possible exception of $10_{161}$ ), we believe that most of the knots must have an equivalent fat diagram presentation. In fact, many complicated higher crossing knot invariants looks calculable when presented as double fat graphs. Particularly, the knots in ref. [43, 44] belongs to family of starfish diagram with three-fingers. We will see that there are plenty of mutants within the starfish family, which belong to the familiar class called pretzel knots [49]. Interestingly, we can calculate $R=[21]$ colored HOMFLY polynomials for such mutant pairs and explicitly check whether the mutants are distinguishable or not.

It is appropriate to mention that the eq. (1.2) describing the colored HOMFLY polynomials for the fat tree diagrams is by no means a trivial formula. Particularly, consistency of knot equivalences led to formal theorems for $k$-valent vertex states [50-57]. However, its origins are not immediately clear neither from the conformal blocks [10, 50-57] nor from the Reshetikhin-Turaev [58-69] approaches to knot/link polynomials. There is lot of evidence for the eq. (1.2) from the conformal block method [42-47] and its advanced versions like the evolution [70], cabling [40] and differential expansion [39] methods for symmetric representations. In this paper, we will do similar verification for $R=$ [21]. Moreover, eq. (1.2) looks typical for topological field theories (like the Hurwitz model in [71-73]) and 
it can serve as a basis of a new intuition and calculus in knot theory, involving a kind of pant decomposition of link diagrams.

The paper is organized as follows. In the first part we describe the elementary building blocks for double far graphs and explain how to construct knots out of them. In the second part we first describe some peculiarities of the representation theory especially related to the first non-trivial mixed representation $R=[21]$, and then consider the colored HOMFLY polynomials in representation $R=[21]$ for various knots. In particular, we check that these HOMFLY polynomials do differ between the notorious Conway-Kinoshita-Terasaka (KTC) mutant pair, in accordance with the expectations [74]. Moreover, the manifest difference between their HOMFLY polynomials is in complete agreement with [75]. We also discuss other mutant pairs. In particular, we find a difference between the HOMFLY polynomials for a whole 2-parametric family of mutants. However, representation $R=[21]$ is not sufficient to distinguish between another set of mutants. We list explicit [21 colored HOMFLY polynomials for various knots in the appendix. We have a table that describes the features of all knots upto 10 crossings.

Note that all answers for the HOMFLY polynomials in representation $R=[21]$ in this paper for the knots that have a three braid representation we compared with the results obtained by the cabling method of $[37,40]$. Besides, we made a few self-consistency checks, see section 7.4.

Throughout the paper we use the notation $[n]$ both for the quantum number and for the representation (e.g., [21]). Hopefully this would not lead to a misunderstanding. Other notations are

$$
D_{i}=\frac{A q^{i}-A^{-1} q^{-i}}{q-q^{-1}} \quad\{x\}=x-\frac{1}{x}
$$

and $d_{R}$ is the quantum dimension of the representation $R$.

\section{Part I}

\section{Diagrammar}

\section{Chern-Simons evolution}

Expression (1.6) for each propagator (1.5) in (1.2) is usually interpreted as the ordinary time evolution in Chern-Simons theory, which is provided by monodromies of conformal block made from braiding matrices $T_{ \pm}, T_{0}$ which are of three types (depending on the pair of adjacent strands that it acts on) and Racah matrices $S$, see [10] for the original idea and $[43-47,50-57]$ for recent applications. In the case of arbitrary representation $R$, the form of the $S$ matrix and the eq. (1.6) needs to be formulated accurately. This section describes our notation, which will be used in the rest of the text.

$S$ is a matrix, with two peculiar multi-indices $X a b$ and $Y c d$, which are collections of square matrices of different sizes, depending on irreducible representations $X$ and $Y$. Two 
more potentially convenient notation are

$$
S_{Y c d, X a b}^{1234}=S[\overbrace{X, a}^{\overbrace{R_{1}, R_{2}}^{Y, c}} \mid \underbrace{\bar{Y}, d}_{\bar{X}, b}]=S_{X a b}^{Y c d}\left[\begin{array}{cc}
R_{1} & R_{2} \\
R_{3} & R_{4}
\end{array}\right]:
$$

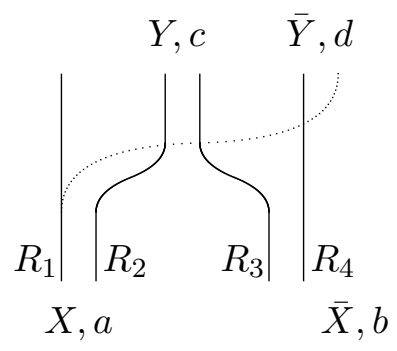

The punctured line helps to illustrate the meaning of indices, it indicates that, after applying the Racah matrix $S$, the representation $R_{1}$ becomes close to $R_{4}$. Note that the initial state $X a b$ stands to the right/bottom of the final $Y c d$, but representations are ordered oppositely: from the left to the right and from the top to the bottom. When we write explicit matrices in section 6 , the left/upper indices (like $Y c d$ ) label rows, while the right/bottom $(X a b)$ - columns.

The composition of two evolutions gives the identity:

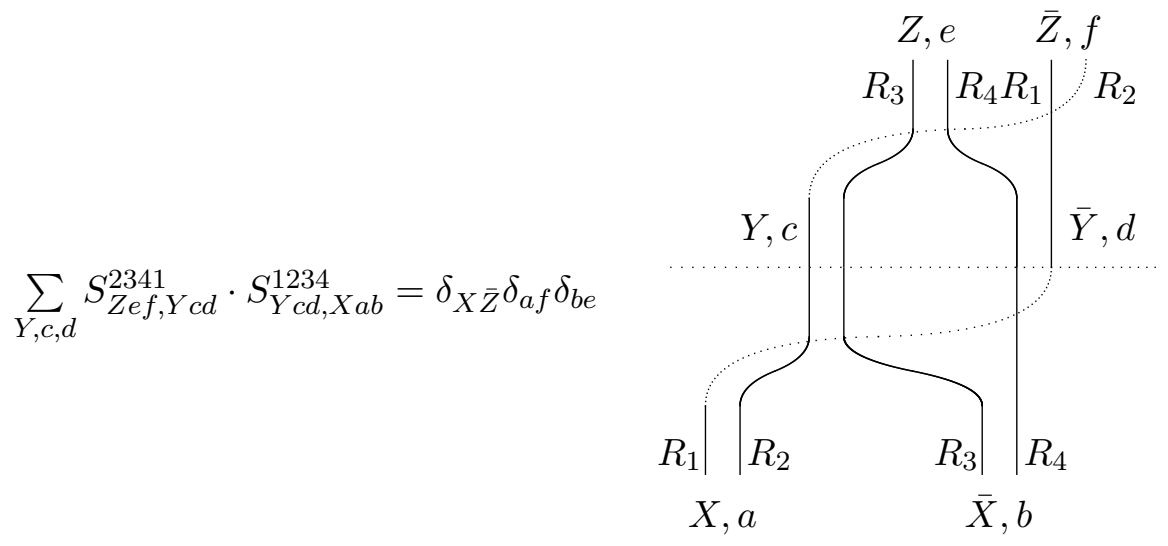

If combined with unitarity of $S$ (orthogonality when $S$ is real),

$$
\sum_{Y, c, d} S_{Y c d, Z e f}^{1234} \cdot\left(S^{1234}\right)_{Y c d, X a b}^{*}=\delta_{X Z} \delta_{a e} \delta_{b f}
$$

this implies:

$$
S_{Z e f, Y c d}^{2341}=S_{Y c d, \bar{Z} f e}^{1234}
$$

To study knots, we require two of the four representations $R_{1}, R_{2}, R_{3}, R_{4}$ to be $R$ and the other two to be conjugate representation $\bar{R}$. This implies that there are two independent matrices, which we denote $S$ and $\bar{S}$ :

$$
\begin{aligned}
& S_{Y c d, X a b}^{R \bar{R} R \bar{R}}=\bar{S}_{Y c d, X a b}, \quad X a b, Y c d \in R \otimes \bar{R}, \\
& S_{Y c d, X a b}^{\bar{R} R \bar{R} R}=S_{X a b, \bar{Y} d c}^{R \bar{R} R \bar{R}}=\bar{S}_{X a b, \bar{Y} d c},
\end{aligned}
$$




$$
\begin{aligned}
& S_{Y c d, X a b}^{R R \bar{R} \bar{R}}=S_{Y c d, X a b}, \\
& S_{Y c d, X a b}^{\bar{R} \bar{R} R R}=S_{X a b, \bar{Y} d c}^{R \bar{R} \bar{R} R}=S_{\bar{Y} d c, \bar{X} b a}, \\
& S_{Y c d, X a b}^{R \bar{R} \bar{R} R}=S_{X a b, \bar{Y} d c}^{R R \bar{R} \bar{R}}=S_{X a b, \bar{Y} d c}, \\
& S_{Y c d, X a b}^{\bar{R} R R \bar{R}}=S_{X a b, \bar{Y} d c}^{\bar{R} \bar{R} R}=S_{\bar{X} b a, Y c d}
\end{aligned}
$$$$
X a b \in R \otimes R, Y c d \in R \otimes \bar{R}
$$

Note that $S$ converts two parallel braids into two antiparallel, while $\bar{S}$ converts antiparallel into antiparallel:

$$
\begin{array}{|ccc|}
\hline \text { antiparallel } \stackrel{\bar{S}=\bar{S}^{\dagger}}{\longleftarrow} \text { antiparallel } \\
\text { antiparallel } \stackrel{S}{\stackrel{S}{L}} \text { parallel } \\
\text { parallel } \quad \stackrel{S^{\dagger}}{\longleftarrow} \text { antiparallel }
\end{array}
$$

Operators $T$ describe crossing of two adjacent strands and are much simpler than $S$. The only delicate point is that there are three different pairs of adjacent strands in the 4strand braid and therefore there are three different $T$-matrices, which we denote $T^{+}, T^{0}$ and $T^{-}$. Also, the two intersecting strands can be either parallel or antiparallel, what we denote by $T$ and $\bar{T}$ respectively. This brings the number of different $T$-insertions in (1.6) to six.

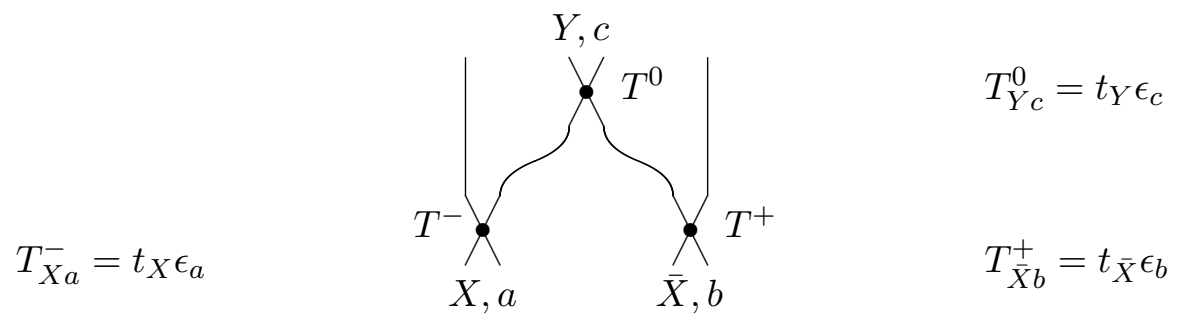

One can promote these operators to matrices of the same type as $S$ :

$$
\begin{aligned}
& T_{X a b, X^{\prime} a^{\prime} b^{\prime}}^{-}=t_{X} \epsilon_{a} \cdot \delta_{X X^{\prime}} \delta_{a a^{\prime}} \delta_{b b^{\prime}} \\
& T_{X a b, X^{\prime} a^{\prime} b^{\prime}}^{0}=t_{X} \epsilon_{a} \cdot \delta_{X X^{\prime}} \delta_{a a^{\prime}} \delta_{b b^{\prime}} \\
& T_{X a b, X^{\prime} a^{\prime} b^{\prime}}^{+}=t_{X} \epsilon_{b} \cdot \delta_{X X^{\prime}} \delta_{a a^{\prime}} \delta_{b b^{\prime}}
\end{aligned}
$$

The eigenvalues are the standard ones in the theory of cut-and-join $\hat{W}$-operators $[72,73]$ and the same which appear in the Rosso-Jones formula [13, 76, 77]:

$$
\left\{\begin{array}{c}
t_{X \in R \otimes R} \\
\bar{t}_{X \in R \otimes \bar{R}}
\end{array}\right\}=\frac{\epsilon_{X}}{A^{|R|} q^{2 \varkappa_{R}}} \cdot q^{\varkappa_{X}} \varkappa_{X}=\sum_{(i, j) \in X}(i-j)
$$

All $\epsilon_{X}$ 's take values \pm 1 .

We usually omit the indices, \pm 0 of $T$ later on, since these $T$ 's are all the same as matrices. 


\section{Pretzel fingers and propagators}

Our next goal is to classify different types of expressions (1.6) for the propagator (1.5) - according to the possible number and parities of parameters $n_{\alpha}, m_{\alpha}$ and $l_{\alpha}$ and to directions of strands (arrows). A special role is played by the terminal branches in the tree (1.3), which we call fingers. They are represented by matrix elements $\mathcal{A}_{1 X}^{a b}=\mathcal{A}_{1, X a b}$, and all the $T$-matrices $(X=1)$ standing to the very left of all $S$ can be omitted. According to (2.5), the last (most left) $S$ in such matrix element can be either $\bar{S}$ or $S$ but not $S^{\dagger}$.

In this section we begin from the simplest possible types of propagators and fingers, belonging to the pretzel type. For pretzels all $m_{\alpha}=l_{\alpha}=0$ and there is just one parameter $n$ in each of the $k$ of the fingers. Still, there are propagators and thus fingers of three different kinds, depending on directions of arrows and the parity of $n$. In the case of pretzels, the notation with bars is sufficient to distinguish between all these cases, still we add explicit indices par, ea oa (depending on parallel oriented braids, antiparallel oriented even braids or antiparallel oriented odd braids) to avoid any confusion:

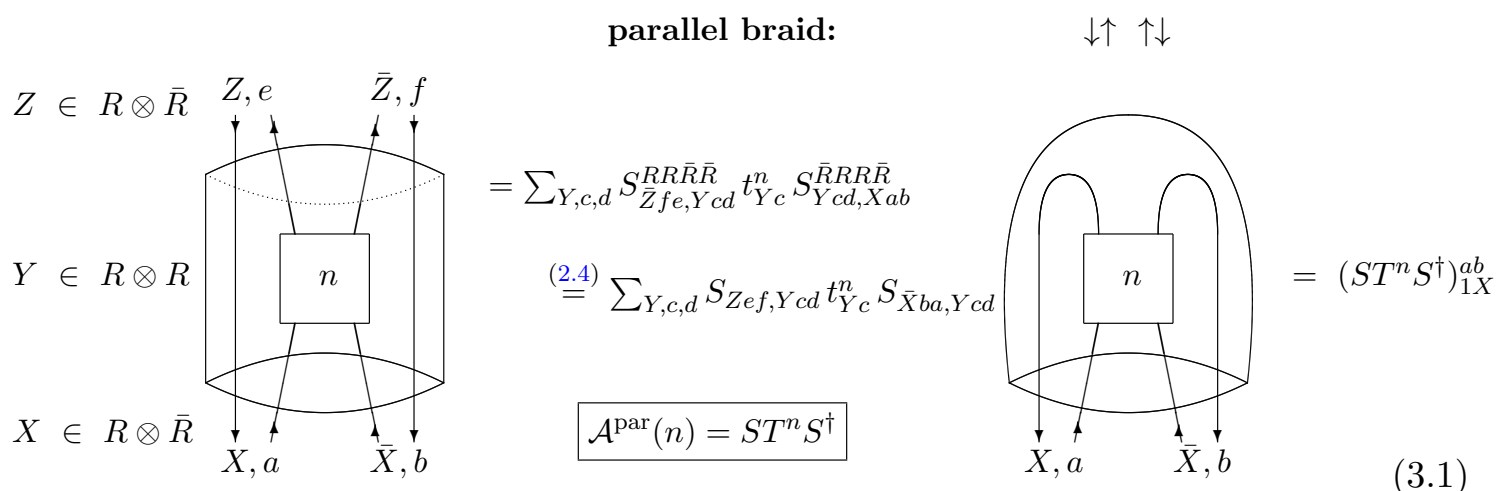

even antiparallel braid: $\quad \downarrow \uparrow \downarrow \uparrow$

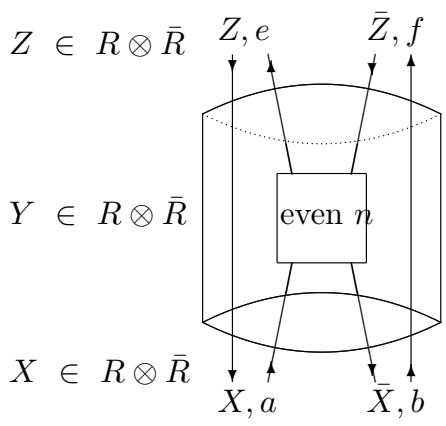

$=\sum_{Y, c, d} S_{\bar{Z} f e, Y c d}^{R \bar{R} R \bar{R}} \bar{t}_{Y c}^{n} S_{Y c d, X a b}^{\bar{R} R \bar{R} R}$

$\stackrel{(2.4)}{=} \sum_{Y, c, d} \bar{S}_{Z e f, Y c d} \bar{t}_{Y c}^{n} \bar{S}_{\bar{X}_{a b}, \bar{Y} d c}$

$\mathcal{A}^{\mathrm{ea}}(\bar{n})=\bar{S} \bar{T}^{n} \bar{S}$

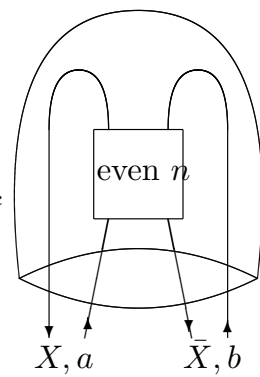

$=\left(\bar{S} \bar{T}^{n} \bar{S}\right)_{1 X}^{a b}$

odd antiparallel braid: $\quad \downarrow \downarrow \uparrow \uparrow$

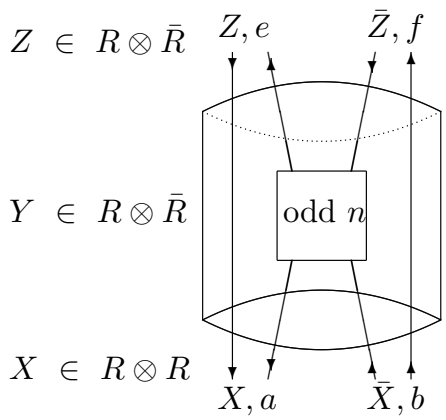

$$
\begin{gathered}
=\sum_{Y, c, d} S_{\bar{Z} f e, Y c d}^{R \bar{R} R \bar{R}} \bar{t}_{Y c}^{n} S_{Y c d, X a b}^{\bar{R} \bar{R} R R} \\
\stackrel{(2.4)}{=} \bar{S}_{Z e f, Y c d} \bar{t}_{Y c}^{n} S_{Y c d, X a b} \\
\mathcal{A}^{\text {oa }}(n)=\bar{S} \bar{T}^{n} S
\end{gathered}
$$

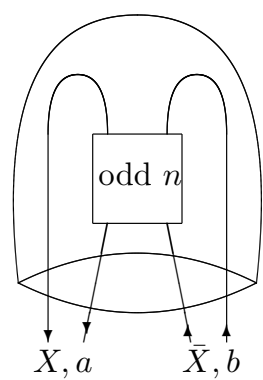

$=\left(\bar{S} \bar{T}_{0}^{n} S\right)_{1 X}^{a b}$ 
The choice of $S$-matrices in above examples is easy to understand from (2.5). Recall, $S$ converts parallel states (from $R \otimes R$ ) into antiparallel (from $R \otimes \bar{R}$ ), while $S^{\dagger}$ does the reverse conversion, and $\bar{S}$ relates antiparallel to antiparallel states.

In the final expressions (boxed) we suppressed most indices, thus implying that we deal with matrices in extended space, where basis is labeled by the multi-index $X a b$. We explain the details of this formalism in section 6 , while in section 5 we use the symbolical notation: just $X$ for the multi-index and $\overline{\sum_{X}}$ for appropriate contractions, including nontrivial sign-factors $\sigma$ (which is symbolized by the bar over the sum).

These are the propogators which are relevant for the knots belonging to pretzel family and the knots in the present paper.

\section{Other building blocks}

\subsection{Non-pretzel fingers}

Generalization to arbitrary non-pretzel fingers and propagators (1.5) with arbitrary parameters $m_{\alpha}, l_{\alpha}, n_{\alpha}$ is exhaustively described by (1.6), one just needs to put appropriate $S$ and $T$ matrices for the given choice of arrows, like we did for the pretzel fingers in section 3 . Since this is straightforward we do not provide this additional list.

What is more important, some diagrams, which do not a priori look like (1.5), actually belong to this family, and this is the reason for the double fat graph description to present so many different knots and links. In this section, we mention just two examples of this kind, exploited in our further considerations.

\subsection{Horizontal loop}

The first example is simple:
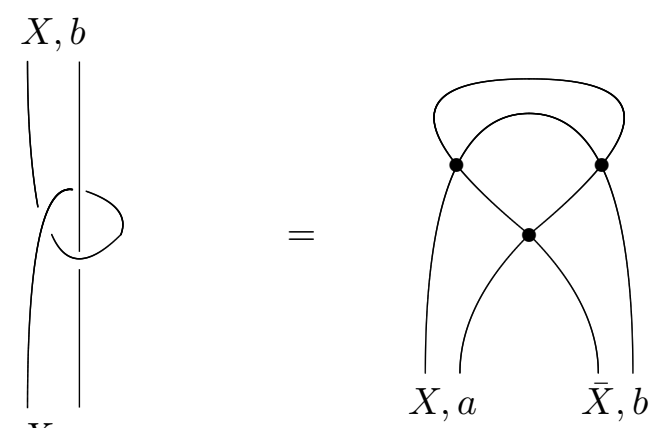

$$
\mathcal{A}^{\mathrm{hl}}=\left(S T_{-} T_{+} S^{\dagger} T_{0} S\right)_{1 X}^{a b}
$$

It is nearly obvious that this is just the non-pretzel finger with parameters $n_{1}=m_{2}=$ $l_{2}= \pm 1$ (signs and the types of $S$ and $T$ matrices depend on the types of intersection and directions of arrows). No $S$-matrix identities are needed to get an expression for it. 
One could easily insert a horizontal braid of arbitrary length and consider a sequence of such horizontal loops:

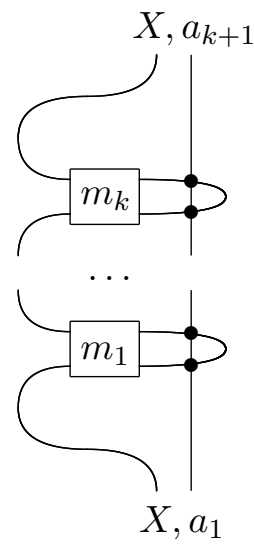

$$
\mathcal{A}^{\mathrm{hl}}\left(m_{1}, \ldots, m_{k}\right)_{X}^{a_{1} a_{k+1}}=\sum_{a_{2}, \ldots, a_{k}} \prod_{i=1}^{k}\left(S T_{-} T_{+} S^{\dagger} T_{0}^{m_{i}} S\right)_{1 X}^{a_{i} a_{i+1}}
$$

\subsection{Horizontal braid}

The isolated horizontal braid

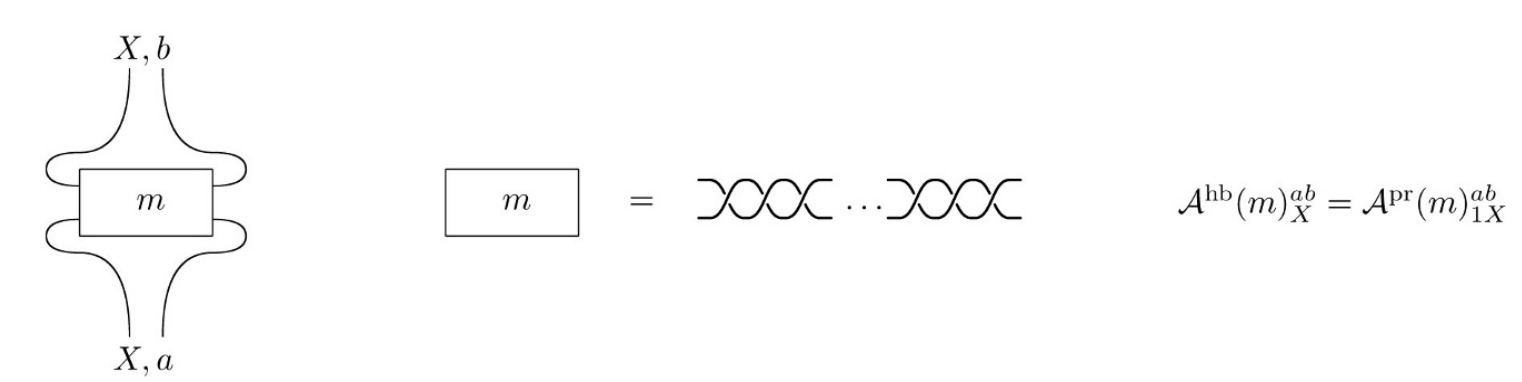

is nothing but the pretzel finger. The type of the finger depends on orientation of lines and the parity of $m$.

More interesting is the horizontal braid located in between the two lines in the double fat propagator as shown:
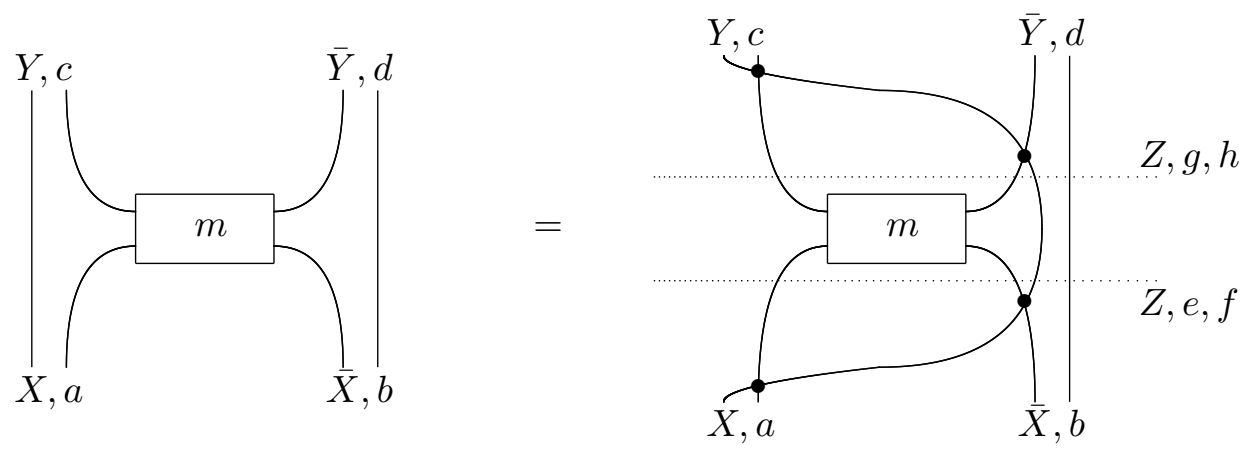

This is a contraction of three blocks, where the middle one is exactly the horizontal braid above. Schematically, it is

$$
\sum_{Z} \frac{1}{\sqrt{d_{Z}}}\left(T^{-1} S^{\dagger} T^{-1} S\right)_{Y Z} \mathcal{A}^{\mathrm{hb}}(m)_{1 Z}\left(T S^{\dagger} T S\right)_{X Z} \stackrel{\stackrel{(6.4)}{=}}{=} \sum_{Z} \frac{1}{\sqrt{d_{Z}}} S_{Y Z} S_{X Z} \mathcal{A}_{1 Z}^{\mathrm{pr}}(m)
$$


In fact there are seven versions of this relation, for different arrow directions and different parities of $m$. Four of them describe even $m$ :
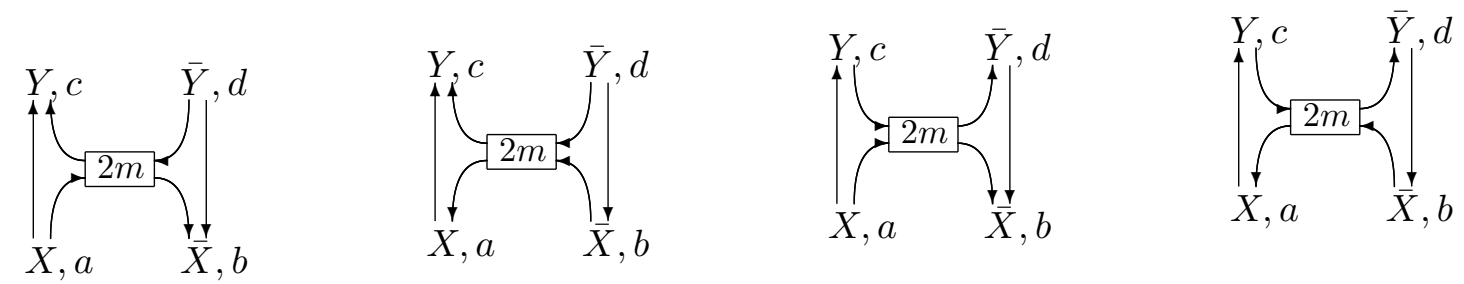

These graphs are described by

$$
\sum_{Z \in R \otimes \bar{R}} \frac{1}{\sqrt{d_{Z}}} \bar{S}_{Y Z} \bar{S}_{X Z} \mathcal{A}_{1 Z}(2 m)
$$

with $\mathcal{A}_{1 Z}(2 m)$ being $\mathcal{A}^{\text {ea }}$ in the first and the fourth pictures and $\mathcal{A}^{\text {par }}$ in the two remaining pictures.

Similarly, odd $m$ are described by the following four pictures:
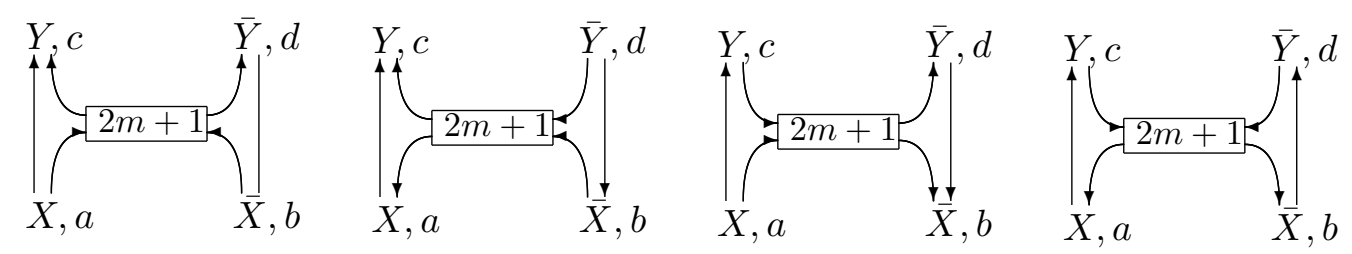

The first graph here does not emerge as a subgraph of the tree double fat diagrams, and the three remaining are described by

$$
\sum_{Z \in R \otimes \bar{R}} \frac{1}{\sqrt{d_{Z}}} \bar{S}_{Y Z} \bar{S}_{X Z} \mathcal{A}_{1 Z}(2 m+1)
$$

with $\mathcal{A}_{1 Z}(2 m+1)$ being $\mathcal{A}^{\text {par }}$ in the second and the third pictures and $\mathcal{A}^{\text {oa }}$ in the fourth one.

One can perform the simple self-consistency checks by attaching (1.11) and (1.12) to the top and bottom of these configurations.

\section{Some examples of double fat diagrams and knot polynomials}

Contractions of the pretzel fingers produce a wide variety of the pretzel link and knots. We also look at knots obtained by inclusion of non-pretzel fingers with non-vanishing $m_{\alpha}$ and/or $l_{\alpha}$, non-trivial propagators with horizontal braids and the appropriate contractions of the fingers.

It is not our goal in the present paper to provide a systematic description of the variety of double fat diagrams. Instead we demonstrate how knot polynomials are built, when the diagram is given in this form. We present the polynomials for many knots which belongs to the knot families presented in the following subsections. 


\subsection{Pretzel knots and links}

The simplest subfamily inside the tree double fat set is that of the pretzel links/knots, for which eq. (1.2) was analyzed in great detail in [45-47]. If considered as made from the two-strand braids, the pretzel link/knot looks like a $(g=k-1)$-loop diagram naturally lying on the surface of genus $g$ :

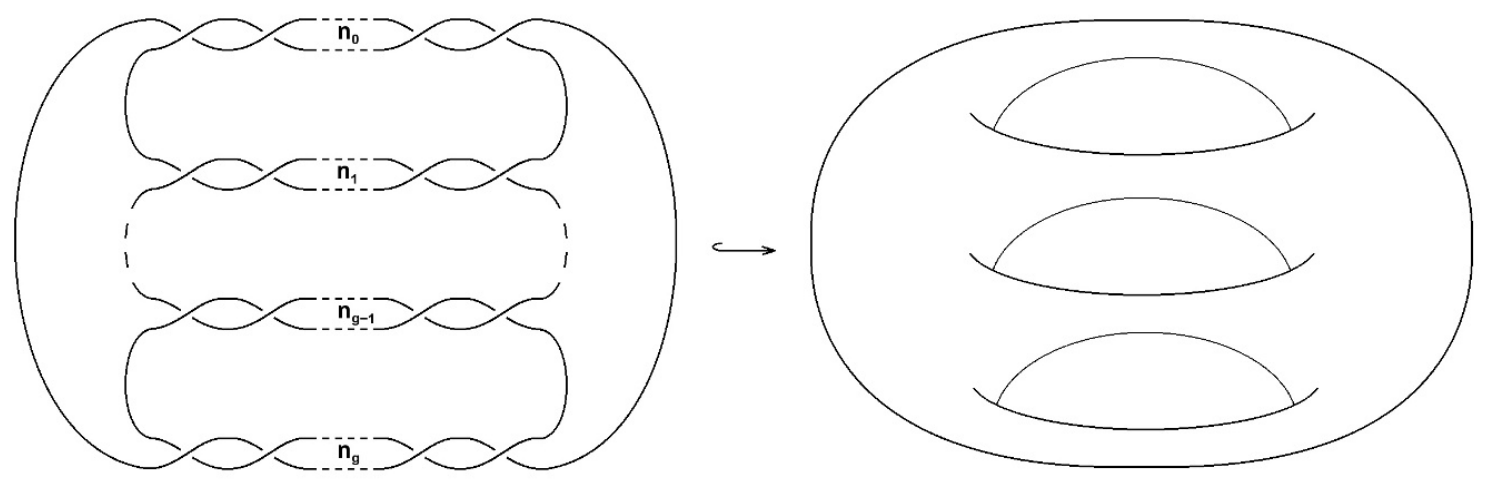

However, if composed from the four-strand braids, pretzel links/knots become trees of type (1.3), with just a single vertex, i.e. are of a peculiar starfish/fingerfish type (it is not a hedgehog, because all the fingers are cyclically ordered):

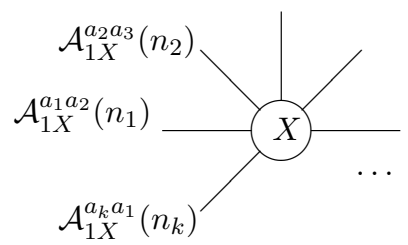

$$
H_{R}^{r e d}=d_{R}^{k-1} \cdot \sum_{X,\{a\}}^{\sigma} d_{X}^{1-k / 2} \prod_{i=1}^{k} \mathcal{A}_{1 X}^{a_{i} a_{i+1}}\left(n_{i}\right) \equiv d_{R}^{k-1} \sum_{X} d_{X}^{1-k / 2} \prod_{i=1}^{k} \mathcal{A}_{1 X}\left(n_{i}\right)
$$

It is clear that parallel and even antiparallel fingers can be combined in arbitrary starfish combinations connected by the states $X \in R \otimes \bar{R}$ while if one finger is odd antiparallel, all connections are via $X \in R \otimes R$, thus all the other fingers should also be odd antiparallel.

Pretzel family contains quite a lot of interesting knots and links. Mutations permute different parameters $n_{i}$, but colored HOMFLY in all (anti)symmetric and rectangular representations, when there are no indices $a_{i}$ are invariant under all permutations of $n_{i}$. In nonrectangular representations dependence of propagators and fingers on the indices $a_{i}$ forces the invariant to be unchanged only for the cyclic permutation which we detail in section 7 .

However, these details do not affect the 3-finger pretzel knots, of which the simplest subfamily is that of the twist knots $T w_{k}$, made from three antiparallel fingers, two of them being of unit length: $T w_{k}=P r_{\overline{1}, \overline{1}, \overline{2 k-1}}$ (clearly, there are no non-trivial mutations in this case). The simplest among the twist knots is the trefoil, which is also a torus 2-strand knot.

\subsubsection{Trefoil}

Trefoil is the pretzel knot $(\overline{1}, \overline{1}, \overline{1})$, thus it has a three-finger representation

$$
\begin{aligned}
H_{R}^{3_{1}} & =d_{R}^{2} \sum_{X \in R \otimes R} d_{X}^{-1 / 2}(\bar{S} \bar{T} S)_{1 X}^{3} \stackrel{(6.4)}{=} d_{R}^{2} \sum_{X} d_{X}^{-1 / 2}\left(\bar{T}^{-1} S T^{-1}\right)_{1 X}^{3}=d_{R}^{2} \sum_{X} \frac{S_{1 X}}{d_{X}^{1 / 2}} \cdot S_{1 X}^{2} T_{X}^{-3}= \\
\stackrel{(1.10)}{=} & d_{R} \sum_{X \in R \otimes R} S_{1 X}^{2} T_{X}^{-3}=d_{R} \cdot \operatorname{Tr} S^{\dagger} T^{-3} S
\end{aligned}
$$

where at the r.h.s. we have the familiar formula for the two-strand knot. 


\subsubsection{Twist knots: thin, triple-boundary (pretzel) $(\overline{1}|\overline{1}| \overline{2 k-1})$}

The twist knots are the closures of braids where the number of strands grows linearly with the number of intersections, and thus braid representation is inconvenient for looking for generic formulas.

Instead, they possess a two-finger (i.e. two-bridge) realization so that

$$
H^{T w(k)}=\left(\bar{S} \bar{T}^{2 k} \bar{S}^{\dagger} \bar{T}^{2} \bar{S}\right)_{00}
$$

and the evolution in $k$ can be straightforwardly studied in this formula in various representations $[70,78]$.

Remarkably, the same family possesses a three-finger (pretzel) realization, with all three fingers odd antiparallel, which is more interesting for the purposes of the present text:

$$
H^{(\overline{1,1,2 k-1})}=d_{R}^{2} \sum_{X \in R \otimes R} d_{X}^{-1 / 2}(\bar{S} \bar{T} S)_{1 X}(\bar{S} \bar{T} S)_{1 X}\left(\bar{S} \bar{T}^{2 k-1} S\right)_{1 X} \stackrel{(6.4)}{=} d_{R}^{2} \sum_{X \in R \otimes R} \frac{S_{1 X}}{d_{X}^{1 / 2}} \cdot S_{1 X} T_{X}^{-2}\left(\bar{S} \bar{T}^{2 k-1} S\right)_{1 X}=
$$

$\stackrel{(1.10)}{=} d_{R} \sum_{X \in R \otimes R} S_{1 X} T_{X}^{-2}\left(S^{\dagger} \bar{T}^{2 k-1} \bar{S}\right)_{X 0}=d_{R} \cdot\left(S T^{-2} S^{\dagger} \bar{T}^{2 k-1} \bar{S}\right)_{00} \Longrightarrow d_{R} \cdot\left(\bar{T} \bar{S} \bar{T}^{2} \bar{S} \bar{T}^{2 k} \bar{S}\right)_{00} \stackrel{\bar{T}_{00}=1}{=} d_{R} \cdot\left(\bar{S} \bar{T}^{2} \bar{S} \bar{T}^{2 k} \bar{S}\right)_{00}$

This is exactly the same as (5.3). The boxed equality in the second line is the double application of (6.4) in the form

$$
T^{-1} S^{\dagger}=S^{\dagger} \bar{T} \bar{S} \bar{T} \quad \Longrightarrow \quad S T^{-2} S^{\dagger}=\overbrace{S S^{\dagger}}^{I} \bar{T} \bar{S} \bar{T}^{2} \bar{S} \bar{T}=\bar{T} \bar{S} \bar{T}^{2} \bar{S} \bar{T}
$$

\subsubsection{Odd antiparallel pretzel knots}

More generally, if there is any odd number of odd antiparallel fingers,

$H_{R}^{P r\left(\bar{n}_{1}, \ldots \bar{n}_{2 k+1}\right)}=d_{R}^{2 k} \overline{\sum_{X \in R \otimes R}} d_{X}^{\frac{1}{2}-k} \mathcal{A}_{1 X}^{\mathrm{oa}}\left(\bar{n}_{1}\right) \ldots \mathcal{A}_{1 X}^{\mathrm{oa}}\left(\bar{n}_{2 k+1}\right) \quad$ with $\quad \mathcal{A}_{1 X}^{\mathrm{oa}}(\bar{n})=\left(\bar{S}^{\dagger} \bar{T}^{n} S\right)_{1 X}$

If the number of fingers is even, we get links rather than knots.

\subsubsection{Pure parallel pretzel knots}

If the pretzel knot is made from the parallel fingers only, their number should be even and exactly one length should be even, otherwise we get a link rather than a knot. The answer for the HOMFLY polynomial is

$H_{R}^{P r\left(n_{1}, \ldots n_{2 k}\right)}=d_{R}^{2 k-1} \overline{\sum_{X \in R \otimes \bar{R}}} d_{X}^{1-k} \mathcal{A}_{1 X}^{\mathrm{par}}\left(n_{1}\right) \ldots \mathcal{A}_{1 X}^{\mathrm{par}}\left(n_{2 k}\right) \quad$ with $\quad \mathcal{A}_{1 X}^{\mathrm{par}}(n)=\left(S \bar{T}^{n} S^{\dagger}\right)_{1 X}$

\subsubsection{Mixed parallel-antiparallel pretzel knots}

The remaining family of the pretzel links/knots contains even number of parallel fingers and odd number of antiparallel, which have even lengths. The corresponding HOMFLY polynomial is

$$
H_{R}^{P r\left(n_{1}, \ldots, n_{2 k}, \bar{m}_{1}, \ldots, \bar{m}_{2 l+1}\right)}=d_{R}^{2 k+2 l} \overline{\sum_{X \in R \otimes \bar{R}}} d_{X}^{\frac{1}{2}-k-l} \mathcal{A}_{1 X}^{\mathrm{par}}\left(n_{1}\right) \ldots \mathcal{A}_{1 X}^{\mathrm{par}}\left(n_{2 k}\right) \cdot \mathcal{A}_{1 X}^{\mathrm{ea}}\left(\bar{m}_{1}\right) \ldots \mathcal{A}_{1 X}^{\mathrm{ea}}\left(\bar{m}_{2 l+1}\right)
$$


with

$$
\mathcal{A}_{1 X}^{\mathrm{par}}(n)=\left(S \bar{T}^{n} S^{\dagger}\right)_{1 X} \quad \text { and } \quad \mathcal{A}_{1 X}^{\mathrm{ea}}(\bar{m})=\left(\bar{S}^{\dagger} \bar{T}^{n} \bar{S}\right)_{1 X}
$$

\section{$5.2 \quad$ Non-pretzel fingers}

Ten intersection chiral knot $10_{71}$ has a neat presentation as a three-finger with each finger being non-pretzel type [42]. Using the states for such non-pretzel fingers, the HOMFLY polynomial for this knot is:

$$
H_{R}^{10_{71}}=\overline{\sum_{X}} \frac{d_{R}^{2}}{\sqrt{d_{X}}}\left(\bar{S} \bar{T}^{2} \bar{S} \bar{T}^{-2} \bar{S}\right)_{1 X}\left(S T^{2} S^{\dagger} \bar{T}^{-1} \bar{S}\right)_{1 X}\left(S T^{-3} S^{\dagger} \bar{T}^{-1} \bar{S}\right)_{1 X}
$$

With the help of (6.6) the last matrix element can be changed for

$$
\left(S T^{-3} S^{\dagger} \bar{T}^{-1} \bar{S}\right)_{1 X} \longrightarrow\left(S T^{-2} S^{\dagger} \bar{T}\right)_{1 X}
$$

Note that beyond $S U_{q}(2)$ this is not a matrix identity but both 1.h.s. or r.h.s. of the above equation inserted in eq. (5.10) give the same polynomial invariant.

\subsection{Double fat graph presentation of knots from three-strand braids}

The next example of non-pretzel knots is a large 4-parametric family: consider a closure of the four boxes in the 3 -strand braid as shown:

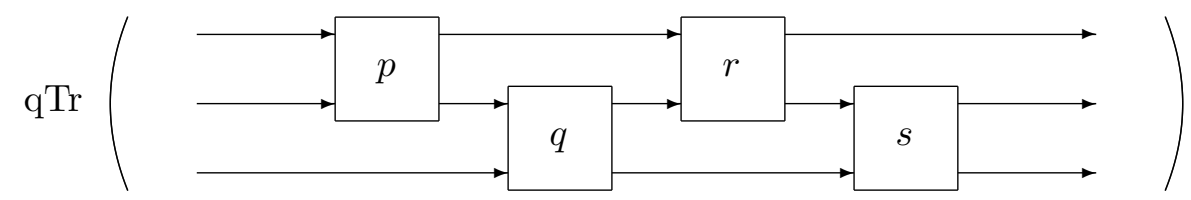

In the fundamental representation the unreduced HOMFLY polynomial is

$$
H_{[1]}^{(p q r s)}=\left(\frac{q}{A}\right)^{p+q+r+s} d_{[3]}+\left(-\frac{1}{q A}\right)^{p+q+r+s} d_{[111]}+d_{[21]} \cdot \operatorname{Tr}_{2 \times 2}\left(S^{\dagger} T^{s} S T^{r} S^{\dagger} T^{q} S T^{p}\right)
$$

In the following subsections, we redraw these four-parametric 3 -strand braid knots as double fat graphs. Particularly, these two equivalent diagrams enables evaluation of knot invariants from both evolution method and from the double fat graph method. 


\subsection{1 $s=1$ : the starfish case}

The choice of one of the four parameters, say $s=1$ gives knots which can be redrawn as a three-finger starfish realization:

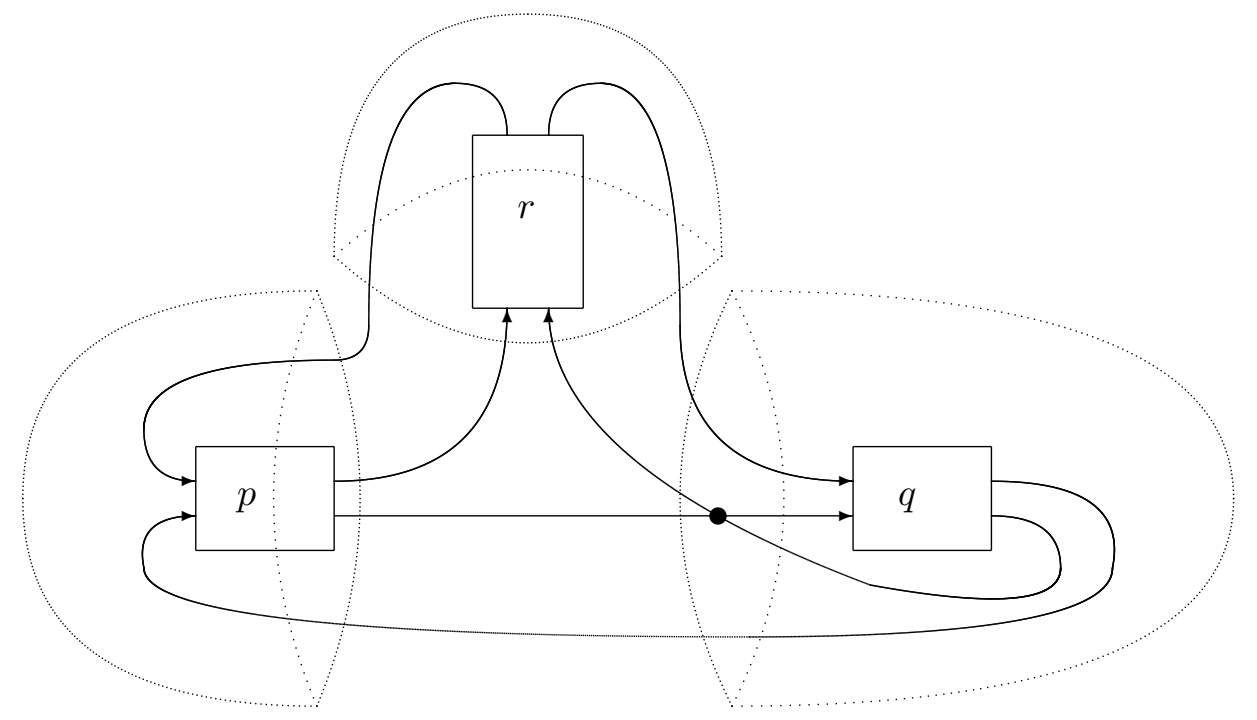

The tree is still (5.1) with just three fingers, but now we allow a non-vanishing parameter $m$ in one of them. In this case in the two fingers with $T^{p}$ and $T^{q}$ we have the antiparallel strands at the entrance converted to the parallel ones and then back, this provides the factors $\left(S T^{p} S^{\dagger}\right)_{1 X}$. In the third finger the antiparallel strands are first converted into antiparallel, then into parallel and then back into the antiparallel ones, this provides the factor $\left(S T^{r} S^{\dagger} \bar{T}^{-1} \bar{S}\right)_{1 X}$ (read from the right to the left):

$$
\begin{aligned}
H_{R}^{(p q r 1)} & =d_{R} \overline{\sum_{X \in R \otimes \bar{R}}} d_{X}^{-1 / 2}\left(S T^{q} S^{\dagger} \bar{T}^{-1} \bar{S}\right)_{1 X}\left(S T^{p} S^{\dagger}\right)_{1 X}\left(S T^{r} S^{\dagger}\right)_{1 X}= \\
& =d_{R} \sum_{X \in R \otimes \bar{R}} d_{X}^{-1 / 2} \overline{\sum_{a, b, c=1}^{m_{X}}} \mathcal{A}(q,-1)_{1 X}^{a b} \cdot \mathcal{A}_{1 X}^{\mathrm{par}}(p)^{b c} \cdot \mathcal{A}_{1 X}^{\mathrm{par}}(r)^{c a}
\end{aligned}
$$

The last two factors in the sum are already familiar $\mathcal{A}^{\mathrm{par}}(p)$ and $\mathcal{A}^{\mathrm{par}}(q)$ from (3.1), while the new one is

$$
\begin{aligned}
\mathcal{A}(q,-1)_{0, X a b} & =\sum_{Z e f, Y c d} S_{0, Z e f}^{R R \bar{R} \bar{R}} t_{Z e}^{q} S_{\bar{Z} f e, Y c d}^{R \bar{R} \bar{R} R} \bar{t}_{Y c}^{-1} S_{Y c d, X a b}^{R \bar{R} R \bar{R}} \\
& \stackrel{(2.4)}{=} \sum_{Z e f, Y c d} S_{0, Z e f} t_{Z e}^{q} S_{Y c d, Z e f} \bar{t}_{Y c}^{-1} \bar{S}_{Y c d, X a b}
\end{aligned}
$$

(like all the sums inside particular fingers, this is an ordinary matrix multiplication without any sign $\sigma$-factors).

The third finger is

$$
\mathcal{A}^{\mathrm{par}_{\overline{1}}}(n)=\mathcal{A}^{\mathrm{par}}(n) \cdot \bar{T}^{-1} \bar{S}=S T^{n} S^{\dagger} \bar{T}^{-1} \bar{S}
$$


and

$$
\overline{\sum_{X, Y}} \frac{d_{[21]}^{3}}{\sqrt{d_{X} d_{Y}}} \mathcal{A}_{1 X}^{\mathrm{par}}\left(n_{1}\right) \mathcal{A}_{1 X}^{\mathrm{par}}\left(n_{3}\right) \bar{S}_{X Y} \mathcal{A}_{0 Y}^{\mathrm{par}}\left(n_{2}\right) \mathcal{A}_{0 Y}^{\mathrm{par}}\left(n_{4}\right)
$$

Configuration (5.14) is not always a knot: it can also be a link with two or even three components:

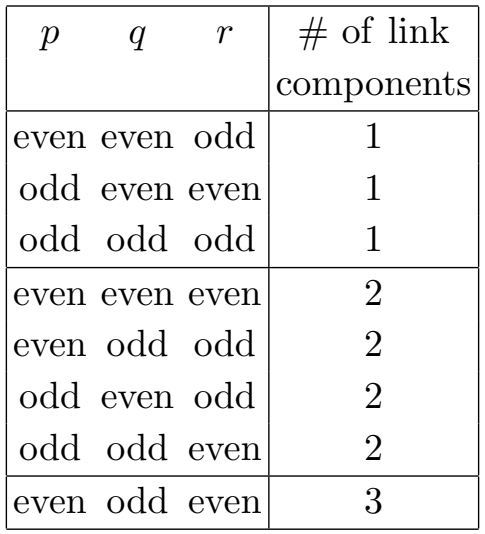

Among knots, the notable members of the three-finger family are the thick knots $10_{124}$ and $10_{139}$, which also have pretzel realizations: $10_{124}=(5,1,3,1)$ and $10_{139}=(4,2,3,1)$.

\subsubsection{Generic four-box 3-strand braids beyond starfish family}

For $s \neq 1$ the braid (5.12) cannot be converted by the Reidemeister moves to a starfish configuration. Instead, it is equivalent to

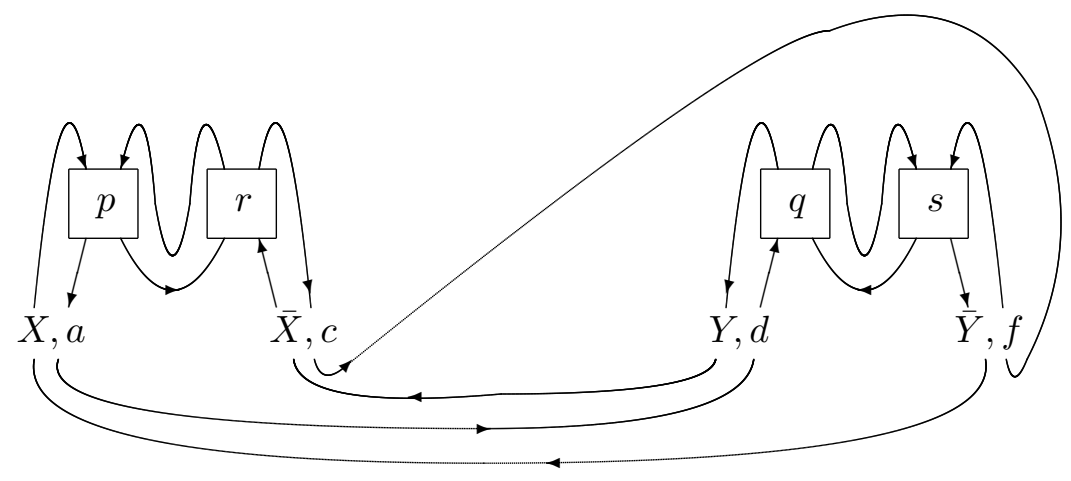

This is actually a double fat tree as shown below:

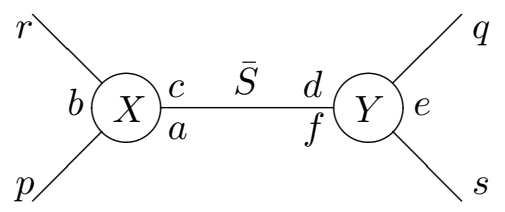

where the propagator (edge), connecting two trivalent vertices, is actually performing an operation like (2.1) on antiparallel strands, i.e. is represented by the matrix $\bar{S}$. According 
to (1.2), the corresponding HOMFLY polynomial is

$$
H^{(p q r s)}=\sum_{X, Y \in R \otimes \bar{R}} \frac{d_{R}^{2}}{\sqrt{d_{X} d_{Y}}} \overline{\sum_{a, b, c=1}^{m_{X}} \sum_{d, e, f=1}^{m_{Y}}} \mathcal{A}^{\mathrm{par}}(p)_{1 X}^{a b} \mathcal{A}^{\mathrm{par}}(r)_{1 X}^{b c} \cdot \bar{S}_{X Y}^{c a, f d} \mathcal{A}^{\mathrm{par}}(q)_{1 Y}^{d e} \mathcal{A}^{\mathrm{par}}(s)_{1 Y}^{e f}
$$

This provides a new type of expression already for $s=1$. The simplest examples of knots with $s \neq 1$ gave 10 intersections: $10_{79}=(3,-2,2,-3)$ and $10_{152}=(3,2,2,3)$.

\subsection{Diagrams with non-tadpole propagators}

This class of knots (5.21) and (5.22) clearly indicates that not all the pretzel fingers are contracted directly. We require an additional propagator connecting two trivalent vertices leading to our basic formula (1.2) with two independent summations. We will call left trivalent vertex as $p p S$ to highlight two fingers with parallel braids and connected by propagator $S$.

\subsubsection{The form for ppS block}

A new building block appearing in (5.22) is

$$
\mathcal{A}_{Y}^{p p S}\left(n_{1}, n_{2}\right)=d_{R} \bar{\sum}_{X \in R \otimes \bar{R}} \frac{1}{\sqrt{d_{X}}} \mathcal{A}_{1 X}^{\mathrm{par}}\left(n_{1}\right) \mathcal{A}_{1 X}^{\mathrm{par}}\left(n_{2}\right) \bar{S}_{X Y}
$$

This expression remains the same if $\bar{S}$ is transposed, $\bar{S}_{X Y} \longrightarrow \bar{S}_{Y X}$.

The number of parallel fingers in this building block can exceed two (but needs to be even), however, for our purposes below, the two will be enough.

For the 4-box 3 -strand braid one can rewrite (5.22) as

$$
\begin{aligned}
H_{R}^{(p q r s)} & =d_{R}^{3} \overline{\sum_{X, Y}} \frac{1}{\sqrt{d_{X} d_{Y}}} \mathcal{A}_{1 X}^{\mathrm{par}}(p) \mathcal{A}_{1 X}^{\mathrm{par}}(r) \bar{S}_{X Y} \mathcal{A}_{1 Y}^{\mathrm{par}}(q) \mathcal{A}_{1 Y}^{\mathrm{par}}(s) \\
& =d_{R}^{2} \overline{\sum_{Y}} \frac{1}{\sqrt{d_{Y}}} \mathcal{A}_{Y}^{p p S}(p, r) \mathcal{A}_{1 Y}^{\mathrm{par}}(q) \mathcal{A}_{1 Y}^{\mathrm{par}}(s)
\end{aligned}
$$

We could have similar tree diagrams with other type of propagators which we discuss now.

\subsubsection{Double braids}

In fact, propagator in (5.22) can be further generalized. One of the important generalization is provided by the horizontal braid (4.3):

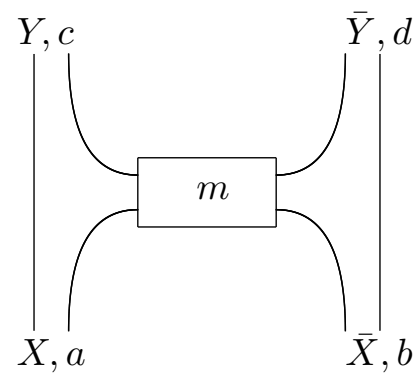

$$
=\sum_{Z} \sum_{e f, g h}^{m_{Z}} \frac{1}{\sqrt{d_{Z}}} \bar{S}_{X Z}^{a b, e f} \bar{S}_{Y Z}^{c d, g h}
$$
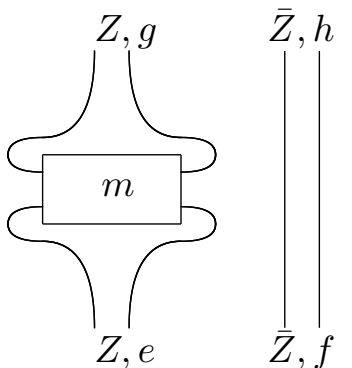
The right two lines in the above diagram do not change the representation, (that is $\delta_{f h}$ ), the left part of this diagram is just the pretzel finger $\mathcal{A}_{1 Z}^{e g}$. The type of $S$-matrices and the pretzel finger depends on the directions of arrows and on the parity of the braid length $m$. The simplest application of (5.25) is to the double braids of [70], which has also the pretzel representation $(-1, \overline{2 k}, m)$. We present more knots using horizontal braids as propagators.

\subsubsection{Pretzel fingers connected via horizontal braid}

We consider examples where pretzel fingers appear from both sides of the horizontal braid:

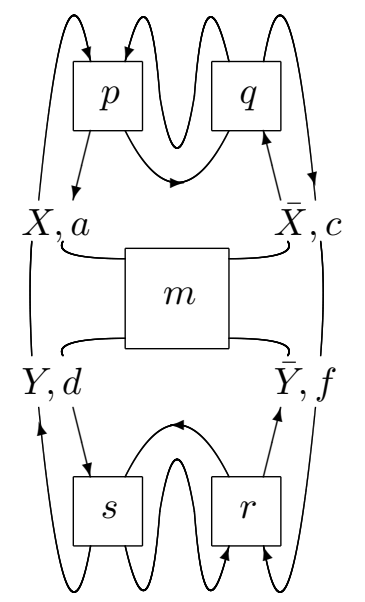

The central block is just the horizontal braid described by the corresponding version of (5.25). Therefore, the reduced HOMFLY polynomial for the diagram (5.26) with even $m$ is

$$
\begin{aligned}
& \sum_{X, Y \in R \otimes \bar{R}} \frac{d_{R}^{2}}{\sqrt{d_{X} d_{Y}}} \sum_{a, b, c=1}^{m_{X}} \sum_{d, e, f=1}^{m_{Y}} \mathcal{A}^{\mathrm{par}}(p)_{1 X}^{a b} \mathcal{A}^{\mathrm{par}}(q)_{1 X}^{b c} \\
\times & \left(\sum_{Z \in R \otimes \bar{R}} \sum_{\overline{g, h, i}}^{\overline{m_{Z}}} \frac{1}{\sqrt{d_{Z}}} \bar{S}_{X Z}^{c a, g h} \mathcal{A}^{\mathrm{ea}}(m)_{1 Z}^{h i} \bar{S}_{Y Z}^{i g, f d}\right) \mathcal{A}^{\mathrm{par}}(r)_{1 Y}^{d e} \mathcal{A}^{\mathrm{par}}(s)_{1 Y}^{e f} \\
= & \sum_{Z} \frac{1}{\sqrt{d_{Z}}} \mathcal{A}_{Z}^{p p S}(p, q) \mathcal{A}_{Z}^{p p S}(r, s) \mathcal{A}_{1 Z}^{\mathrm{ea}}(m)
\end{aligned}
$$

The four external fingers involve parallel braids, while that of the even length $m$ in the propagator is antiparallel. The fat tree is the same (5.21), only the internal propagator is more sophisticated. Mutation is a permutation of $p$ and $q$ or of $r$ and $s$.

\section{Familiar special limits}

- At $m=0$ we get a composite knot, thus the reduced HOMFLY polynomial should factorize. In (5.27) this follows from (1.12), which implies that $\mathcal{A}_{1 Z}^{\mathrm{ea}}(0)=\delta_{1, Z}$.

- At $s=0$ the parallel finger $\mathcal{A}_{1 Y}^{\mathrm{par}}(0)=\delta_{1, Y}$, therefore $\mathcal{A}_{Y}^{p p S}(0, n)=d_{R} \bar{S}_{1 Y} \mathcal{A}_{11}(n)=$ $\sqrt{d_{Y}} \mathcal{A}_{11}(n)$ and

$$
H_{R}^{(p q|m| r 0)}=\mathcal{A}_{11}^{\mathrm{par}}(r) \overline{\sum_{Z}} \mathcal{A}_{1 Z}^{p p S}(p, q) \mathcal{A}_{1 Z}^{\mathrm{ea}}(m)
$$


- If we also take $q=0$, we obtain a composite knot, made out of two 2-strand constituents:

$$
H_{R}^{(p 0|m| r 0)}=H_{R}^{(0 p|m| r 0)}=\mathcal{A}_{11}^{\mathrm{par}}(p) \mathcal{A}_{11}^{\mathrm{par}}(r) \underbrace{\sum_{Z} \sqrt{d_{Z}} \cdot \mathcal{A}_{1 Z}^{\mathrm{ea}}(m)}_{=1}=\mathcal{A}_{11}^{\mathrm{par}}(p) \mathcal{A}_{11}^{\mathrm{par}}(r)
$$

The polynomial form for these double fat graph knots presented in this part I can be worked out for knots carrying fundamental or symmetric representation. This is plausible due to the explicit form of the Racah matrices [79], [45, 2nd paper] known for all symmetric representations. In all these cases all the $\sigma$-factors are unities, and there is no need to deal specifically with overlined sums. The same formula continue to work for representation [21], but then some $\sigma$-factors are -1 . Expressions for $R=$ [21] are rather involved using Racah matrices explicitly calculated in [41]. Our main focus in Part II will be to compute $R=[21]$ colored HOMFLY polynomials.

\section{Part II}

\section{Examples}

Before we proceed with the explicit knot polynomial computations for $R=$ [21], we require expressions for the Racah matrices. We will briefly recapitulate them in the following section.

\section{Racah matrices}

\subsection{Generalities}

The Racah matrices relate the two expansions

$$
\left(R_{1} \otimes R_{2}\right) \otimes R_{3}=\oplus_{P} W_{R_{1} R_{2}}^{P} \otimes(P \otimes R)=\sum_{Q}\left(\oplus_{P} W_{R_{1} R_{2}}^{P} \otimes W_{P R_{3}}^{Q}\right) \otimes Q
$$

and

$$
R_{1} \otimes\left(R_{2} \otimes R_{3}\right)=\oplus_{S} W_{R_{2} R_{3}}^{S} \otimes\left(R_{1} \otimes S\right)=\sum_{Q}\left(\oplus_{S} W_{R_{1} S}^{Q} \otimes W_{R_{2} R_{3}}^{S}\right) \otimes Q
$$

that is, it is a linear operator

$$
\left(\oplus_{S} W_{R_{1} S}^{Q} \otimes W_{R_{2} R_{3}}^{S}\right)=\hat{S}\left[\begin{array}{ll}
R_{3} & Q \\
R_{1} & R_{2}
\end{array}\right]\left(\oplus_{P} W_{R_{1} R_{2}}^{P} \otimes W_{P R_{3}}^{Q}\right)
$$

When the vector spaces $W$ are one-dimensional ("no multiplicities" case), one can represent this linear operator as a matrix with indices $P \in R_{1} \otimes R_{2}$ and $S \in R_{2} \otimes R_{3}$. When $W$ are multidimensional, there is no any distinguished basis and the concrete form of the Racah 


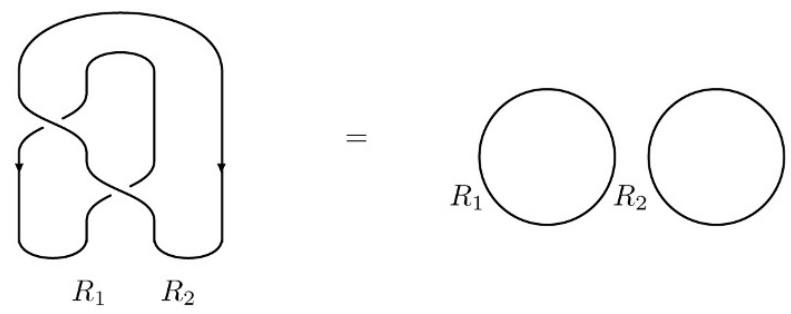

Figure 2. Knot equivalence.

matrix depends on conventions, and on four additional indices labeling the bases in the four $W$-spaces for each given pair $P$ and $S$.

For the purposes of knot (rather than link) theory, all the four representations $R_{1}, R_{2}, R_{3}, Q$ are either $R$ or its conjugate $\bar{R}$. In result, there are two essentially different Racah matrices:

- $S_{S, c, d \mid P, a, b}$ with $P \in R \otimes R$, but $S \in R \otimes \bar{R}$, and

- $\bar{S}_{S, c, d \mid P, a, b}$ with both $P, S \in R \otimes \bar{R}$.

These are the only two kinds of the Racah matrices that show up in our discussion of the double fat tree diagrams. Note that $S$ is essentially asymmetric, while $\bar{S}$ can be symmetric. In fact, $\bar{S}$ is symmetric for $R$ 's taken to be symmetric representation which belongs to the multiplicity-free case.

Another important fact is that the singlet representation $1 \in R \otimes R$, but $1 \notin R \otimes R$ (except for the case of $N=2$ ). Therefore, the matrix elements $1 X$ in (1.2) cannot have $S^{\dagger}$ at the very left, but only $S$ or $\bar{S}$. In general, as we already saw earlier, (2.5) $S$ converts the parallel strands into antiparallel, while $\bar{S}$ antiparallel to antiparallel.

Our fingers never contain three parallel strands, thus these two types of relations are sufficient for our consideration. However, beyond this paper the third Racah matrix connecting the parallel strands to parallel (it was called "mixing matrix" in [64, 65]) plays a big role. Actually, it is much simpler: it does not depend on $N$ (i.e. on $A$ ). For symmetric representations, it coincides with restrictions of both $S$ and $\bar{S}$ to $N=2$, but for more complicated representations the story is a little more involved.

The Racah matrices $S$ are always unitary and satisfy (1.10). There are also additional non-trivial relations like $[41,56]$

$$
\bar{S}^{\dagger} \bar{T} S=\bar{T}^{-1} S T^{-1} \stackrel{\text { conjugation }}{\Longleftrightarrow} S^{\dagger} \bar{T} \bar{S}=T^{-1} S^{\dagger} \bar{T}^{-1}
$$

This identity can be illustrated by figure 2. The knot equivalence gives the following relation:

$$
d_{R_{1}} d_{R_{2}} \cdot\left(S T S^{\dagger} \bar{T} \bar{S}\right)_{11}=d_{R_{1}} d_{R_{2}}
$$

and one can insert $\bar{T}$ to the very left, since it trivially acts on the singlet state. This immediately gives the matrix element $(\ldots)_{11}$ of the second equality of (6.4). 
Further generalization with indices restored (6.4), we get

$$
\sum_{X, a, b, c, d}\left(\bar{S}^{\dagger}\right)_{Y X}^{e f, a b} \bar{T}_{X}^{a b, d c} \bar{S}_{X Z}^{c d, g h}=\sum_{k, l, m, n}\left(T^{-1}\right)_{Y}^{e f, l k} S_{Y Z}^{l k, m n}\left(\bar{T}^{-1}\right)_{Z}^{m n, g h}
$$

An important application of this identity is the possibility of shifting any pretzel finger of length 1 to any position [49] at any representation $R$, though permutations of arbitrary fingers are not a symmetry of the knot.

\subsection{Racah matrices for few symmetric representations}

For symmetric representations $R$, there are no multiplicities, no indices $a, b, c, d$ and the Racah matrices are canonically defined.

- When $R$ is the fundamental representation $R=[1]$, they are given by (1.8) and (1.9), or in a generalizable notation,

$$
S=\frac{1}{\sqrt{[2] D_{0}}}\left(\begin{array}{cc|c}
{[11]} & {[2]} & \\
& & \\
\sqrt{D_{-1}} & \sqrt{D_{1}} & 0 \\
\sqrt{D_{1}} & -\sqrt{D_{-1}} & \text { adj }
\end{array}\right), \quad \bar{S}=\frac{1}{\chi_{[1]}}\left(\begin{array}{cc|c}
0 & \text { adj } & \\
\hline 1 & \sqrt{\bar{d}_{1}} & 0 \\
\sqrt{\bar{d}_{1}} & -1 & \text { adj }
\end{array}\right)
$$

where $D_{i}=\frac{A q^{i}-A^{-1} q^{-i}}{q-q^{-1}}$, and $\bar{d}_{1} \equiv d_{[\overline{1} 1]}=D_{1} D_{-1}, \bar{d}_{2} \equiv d_{[\overline{2} 2]}=\frac{1}{[2]^{2}} D_{3} D_{0}^{2} D_{-1}, \ldots$, $\bar{d}_{m}=D_{2 m-1}\left(\prod_{j=0}^{m-2} \frac{D_{j}}{[j+2]}\right)^{2} D_{-1}$ are the quantum dimensions of the representations from the decomposition $[r] \otimes[r]=1+[\overline{1} 1]+[\overline{2} 2]+\ldots$, where $[\overline{1} 1]$ denotes $\left[21^{N-2}\right]$, $[\overline{2} 2]$ denotes $\left[42^{N-2}\right]$ etc.

- When $R$ is the first symmetric representation $R=[2],[2] \otimes[2]=[4]+[31]+[22]$, $[2] \otimes \overline{[2]}=1+[\overline{1} 1]+[\overline{2} 2]$,

$$
S=\frac{[2]}{D_{1} D_{0}}\left(\begin{array}{ccc|c}
{[22]} & {[31]} & {[4]} & \\
\hline \frac{\chi_{[22]}}{\sqrt{d_{0}}}=\sqrt{\chi_{22}} & \frac{\chi_{31}}{\sqrt{d_{1}}}=\sqrt{\chi_{31}} & \frac{\chi_{4}}{\sqrt{d_{2}}}=\sqrt{\chi_{4}} & 0 \\
\sqrt{\frac{\bar{d}_{1}}{d_{0}}} \cdot \frac{D_{1} D_{0}^{2}}{[2]^{2}[3]} & \sqrt{\frac{\bar{d}_{1}}{d_{1}}} \cdot \frac{\left(D_{2}-D_{0}\right) D_{1} D_{0}}{[2][4]}-\sqrt{\frac{\bar{d}_{1}}{d_{2}}} \cdot \frac{D_{3} D_{1} D_{0}}{[3][4]} & \operatorname{adj} \\
\sqrt{\frac{\bar{d}_{2}}{d_{0}}} \cdot \frac{D_{1} D_{0}}{[2][3]} & -\sqrt{\frac{\bar{d}_{2}}{d_{2}}} \cdot \frac{D_{1} D_{0}}{[4]} & \sqrt{\frac{\bar{d}_{2}}{d_{2}}} \cdot \frac{D_{1} D_{0}}{[3][4]} & {[\overline{2} 2]}
\end{array}\right)
$$

with

$$
\begin{aligned}
& d_{m} \equiv d_{[r+m, r-m]}=\chi_{[r+m, r-m]}=\frac{[2 m+1]}{[r+m+1] ![r-m] !} \prod_{i=0}^{2 r-1} D_{j} \prod_{j=0}^{r-m-1} \frac{D_{j-1}}{D_{r+m+j}}, \\
& r=2
\end{aligned}
$$


and

$$
\bar{S}=\frac{1}{\chi_{[2]}}\left(\begin{array}{ccc|c}
0 & \operatorname{adj} & {[\overline{2} 2]} & \\
\hline 1 & \sqrt{\bar{d}_{1}} & \sqrt{\bar{d}_{2}} & 0 \\
\sqrt{\bar{d}_{1}} & \frac{D_{1}}{[2] D_{2}}\left(D_{3} D_{-1}-1\right) & -\frac{D_{0}}{D_{2}} \sqrt{D_{3} D_{1}} & \operatorname{adj} \\
\sqrt{\bar{d}_{2}} & -\frac{D_{0}}{D_{2}} \sqrt{D_{3} D_{1}} & \frac{D_{0}}{D_{2}} & {[\overline{2} 2]}
\end{array}\right)
$$

- Similarly, for representation $R=[3]$ and so on.

\subsection{Representation [21]}

In the case of $R=[21]$ there are seven different items $X$ in the decomposition

$$
[21] \otimes \underbrace{\overline{[21]}}_{\left[2^{N-2} 1\right]}=\left[432^{N-4} 1\right] \oplus\left[42^{N-2}\right] \oplus\left[42^{N-3} 11\right] \oplus\left[332^{N-3}\right] \oplus\left[332^{N-4} 11\right] \oplus \underline{2 \cdot\left[32^{N-2} 1\right]} \oplus \underbrace{\left[2^{N}\right]}_{\text {singlet }}
$$

The corresponding dimensions and eigenvalues are

\begin{tabular}{|c|c|c|}
\hline$X$ & $d_{X}$ & $t_{X}$ \\
\hline$\left[2^{N}\right]$ & 1 & 1 \\
{$\left[42^{N-3} 11\right]$} & $\frac{[N-2][N-1][N+1][N+2]}{[2]^{2}}$ & $-A^{2}$ \\
{$\left[332^{N-3}\right]$} & $\frac{[N-2][N-1][N+1][N+2]}{[2]^{2}}$ & $-A^{2}$ \\
{$\left[332^{N-4} 11\right]$} & $\frac{[N-3][N]^{2}[N+1]}{[2]^{2}}$ & $-q^{-2} A^{2}$ \\
{$\left[432^{N-4} 1\right]$} & $\frac{[N-3][N-1]^{2}[N+1]^{2}[N+3]}{[3]^{2}}$ & $A^{3}$ \\
{$\left[42^{N-2}\right]$} & $\frac{[N-1][N]^{2}[N+3]}{[2]^{2}}$ & $q^{2} A^{2}$ \\
\hline$\left[32^{N-2} 1\right]$ & $\frac{[N-1][N+1]}{2}$ & $\pm A$ \\
\hline
\end{tabular}

Only one (underlined) item $\left[32^{N-2} 1\right]=\left[21^{N-2}\right]=A d j$ enters (6.11) with non-trivial multiplicity $m_{A d j}=2$, however this makes the Racah matrices $10 \times 10$, since $\sum_{X} m_{X}^{2}=$ $6 \cdot 1+1 \cdot 2^{2}=10$. They are explicitly found in [41]. The pure antiparallel matrix $\bar{S}$ is 


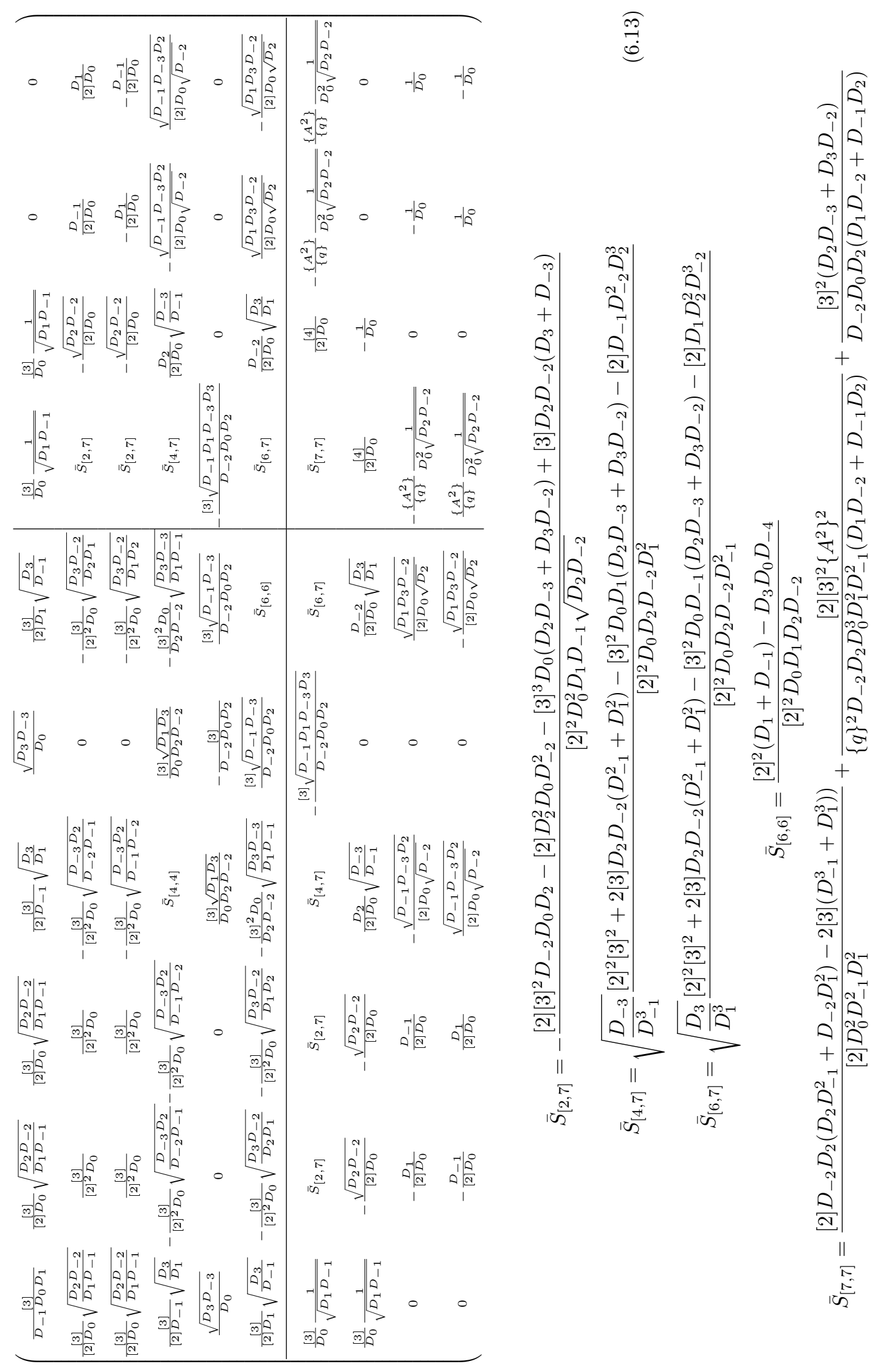


For $S$ we additionally need the expansion ${ }^{1}$

$$
[21] \otimes[21]=[42]+[411]+[33]+\underline{2 \cdot[321]}+[222]+[3111]+[2211]
$$

with

\begin{tabular}{|c|c|c|}
\hline$X$ & $d_{X}$ & $t_{X}$ \\
\hline$[222]$ & $\frac{[N-2][N-1]^{2}[N]^{2}[N+1]}{[2]^{2}[3]^{2}[4]}$ & $q^{-3} A^{-3}$ \\
{$[411]$} & $\frac{[N-2][N-1][N][N+1][N+2][N+3]}{[2]^{2}[3][6]}$ & $-q^{3} A^{-3}$ \\
{$[33]$} & $\frac{[N-1][N]^{2}[N+1]^{2}[N+2]}{[2]^{2}[3]^{2}[4]}$ & $-q^{3} A^{-3}$ \\
{$[2211]$} & $\frac{[N-3][N-2][N-1][N]^{2}[N+1]}{[2]^{2}[4][5]}$ & $-q^{-5} A^{-3}$ \\
{$[3111]$} & $\frac{[N-3][N-2][N-1][N][N+1][N+2]}{[2]^{2}[3][6]}$ & $q^{-3} A^{-3}$ \\
{$[42]$} & $\frac{[N-1][N]^{2}[N+1][N+2][N+3]}{[2]^{2}[4][5]}$ & $q^{5} A^{-3}$ \\
{$[321]$} & $\frac{[N-2][N-1][N]^{2}[N+1][N+2]}{[3]^{2}[5]}$ & $\pm A^{-3}$ \\
\hline
\end{tabular}

Here we again encounter one item with multiplicity two, and the parallel $\rightarrow$ antiparallel Racah matrix $S$ is

\footnotetext{
${ }^{1}$ For comparison, for rectangular representations all multiplicities are unit:

$$
[2] \otimes[2]=[4]+[31]+[22],
$$$$
[22] \otimes[22]=[44]+[431]+[422]+[3311]+[3221]+[2222],
$$

$[33] \otimes[33]=[66]+[651]+[642]+[633]+[5511]+[5421]+[5331]+[4422]+[4332]+[3333]$,
} 


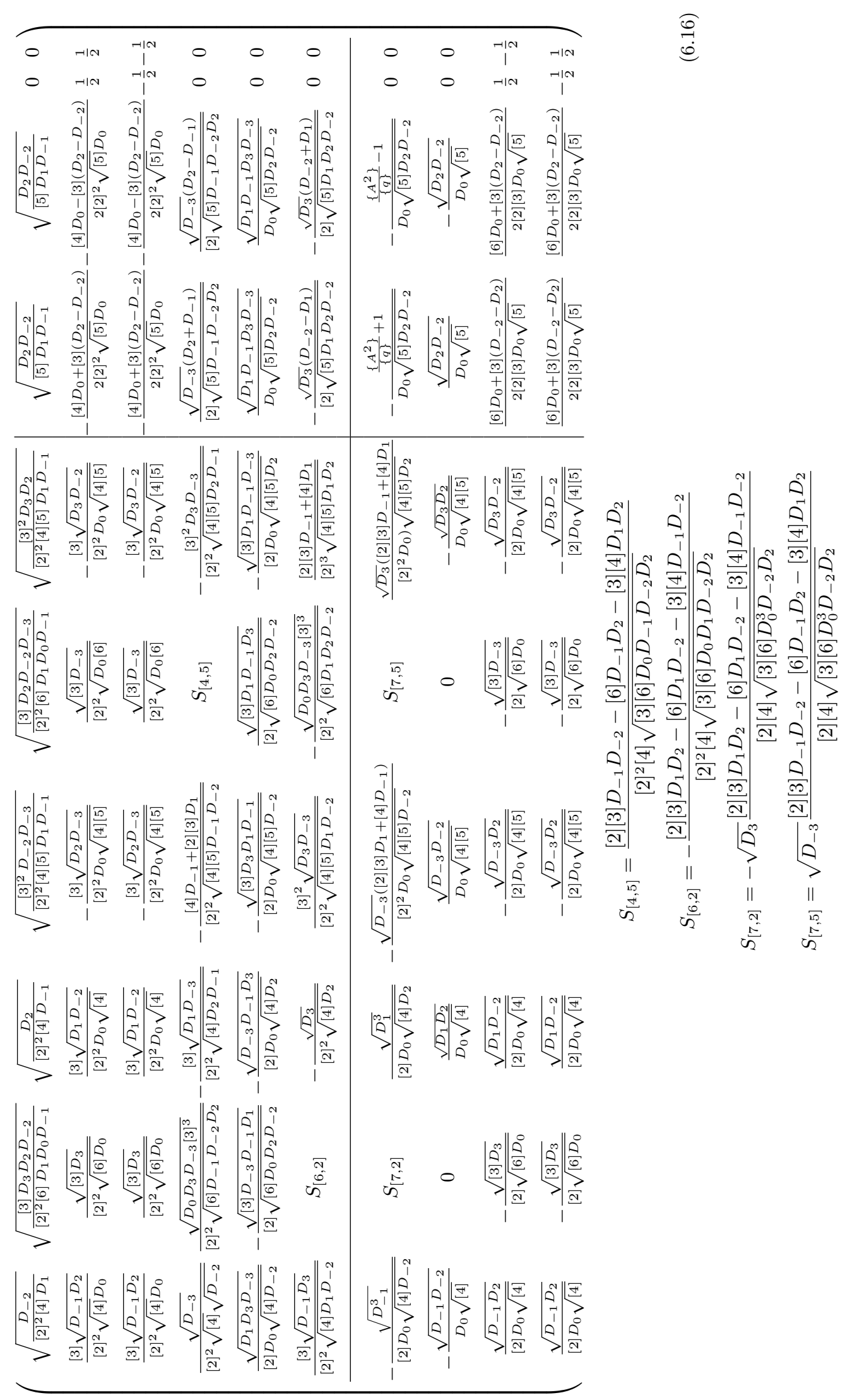


This matrix is obtained from that of [41] by transposition to make it consistent with (1.10) and thus with our representation of knots. Also minor (always allowed) conjugations are performed in (6.13) and (6.16) to get rid of unnecessary minuses and imaginary units.

Diagonalized $\mathcal{R}$-matrices $\bar{T}$ and $T$ are read off from the last columns of (6.12) and (6.15):

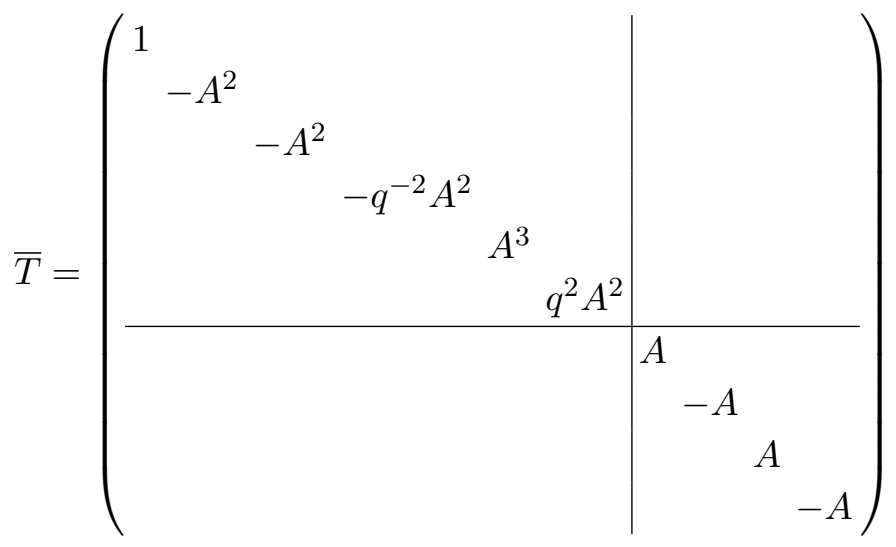

and

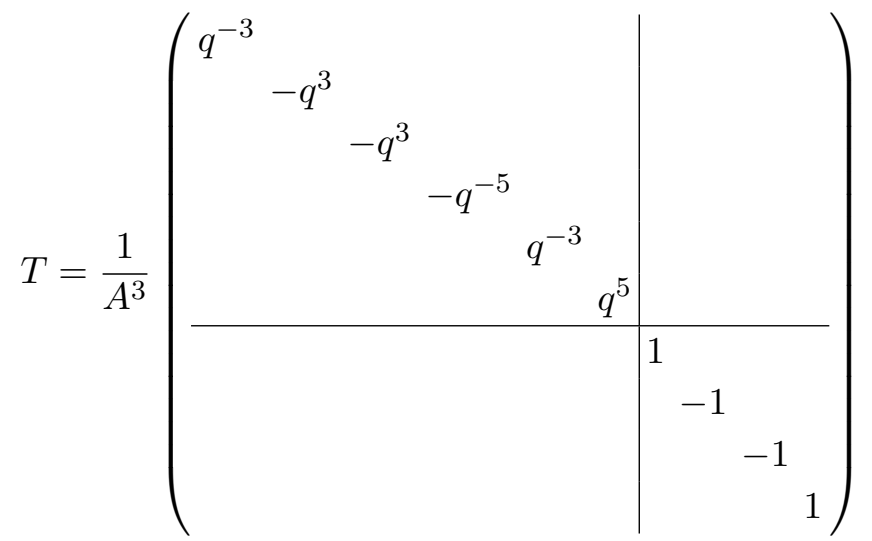

In these $S$ - and $T$-matrices the index $X$ runs from 1 to 7 , and for $X=7$ there are additional indices $a_{i}$ which take two values, which we substitute as $7_{11} \longrightarrow 7,7_{22} \longrightarrow 8,7_{12} \longrightarrow 9$, $7_{21} \longrightarrow 10$, and $d_{10}=d_{9}=d_{8}=d_{7}$. Then contractions in (1.2) for the simplest double-fat graph (5.1) can be rewritten as follows:

$$
\begin{aligned}
& 2 \text { - finger case : } \quad \sum_{X=1}^{7} \sum_{a, b=1}^{m_{X}} A_{X}^{a b} B_{X}^{b a} \quad \longrightarrow\left(\sum_{X=1}^{8} \mathcal{A}_{X} \mathcal{B}_{X}\right)+\left(\mathcal{A}_{9} \mathcal{B}_{10}+\mathcal{A}_{10} \mathcal{B}_{9}\right) \\
& 3 \text { - finger case : } \sum_{X=1}^{7} \sum_{a, b, c=1}^{m_{X}} d_{X}^{-1 / 2} A_{X}^{a b} B_{X}^{b c} C_{X}^{c a} \longrightarrow\left(\sum_{X=1}^{8} d_{X}^{-1 / 2} \mathcal{A}_{X} \mathcal{B}_{X} \mathcal{C}_{X}\right)+d_{7}^{-1 / 2}\left(\mathcal{A}_{7} \mathcal{B}_{9} \mathcal{C}_{10}+\mathcal{A}_{8} \mathcal{B}_{10} \mathcal{C}_{9}+\right. \\
& \left.+\mathcal{B}_{7} \mathcal{C}_{9} \mathcal{A}_{10}+\mathcal{B}_{8} \mathcal{C}_{10} \mathcal{A}_{9}+\mathcal{C}_{7} \mathcal{A}_{9} \mathcal{B}_{10}+\mathcal{C}_{8} \mathcal{A}_{10} \mathcal{B}_{9}\right) \\
& \text { in general } \quad \sum_{X=1}^{7} d_{X}^{1-\frac{k}{2}} \prod_{i=1}^{k} A_{X}^{(i)} \quad \longrightarrow\left(\sum_{X=1}^{8} d_{X}^{1-\frac{k}{2}} \prod_{i=1}^{k} \mathcal{A}_{X}^{(i)}\right)+d_{7}^{1-\frac{k}{2}} \sum_{i \neq j}^{k} \mathcal{A}_{9}^{(i)} \mathcal{A}_{10}^{(j)} \cdot(\ldots)
\end{aligned}
$$

It is the underlined term that breaks the permutation symmetry between different $A^{(i)}$ in the original product down to just cyclic symmetry, and it is the one that distinguishes mutants. When all $\mathcal{A}_{9}^{(i)}$ or $\mathcal{A}_{10}^{(i)}$ are vanishing, the symmetry is preserved and the mutants are not distinguished by the [21]-colored HOMFLY. 


\section{$7 \quad \sigma$-factors and other technicalities}

We emphasize that the eq. (1.2) is a kind of a conjecture/educated guess, and was well tested only for symmetric representations where no multiplicities (indices $a, b, c, \ldots$ ) arise. Hence there is no reason to insist that contractions (6.19) are exactly correct. In fact, they are not: for complicated enough knots, (1.2) with rule (6.19) does not produce polynomials in representation [21] and, in result, fails to reproduce the known answers. Both these problems are cured by switching to $\sigma$, which is allowed to take the values \pm 1 only but not +1 always.

The procedure at the moment is to adjust these sign factors for a given knot so that the answer for HOMFLY is a Laurent polynomial in $A$ and $q$. The choice of sign is unique in some examples. We also observe that there could more than one possible choice of sign giving same polynomial when one of the fingers is pretzel of length one or some matrix elements $\left(\mathcal{A}_{9}^{(i)}\right.$ or $\left.\mathcal{A}_{10}^{(i)}\right)$ simply vanish.

No general rule is found yet for a priori determination of $\sigma$. We have observed the sign pattern for some simple families of double fat diagrams we studied. We present, in the following section, the signs of $\sigma$ which we encountered in obtaining polynomial invariants.

\subsection{Relevant choices}

\subsubsection{3 fingers}

In the simplest case of 3 fingers we use the following notation:

$$
\sum_{X=1}^{7} \overline{\sum_{a, b, c=1}^{m_{X}}} d_{X}^{-1 / 2} A_{X}^{a b} B_{X}^{b c} C_{X}^{c a} \longrightarrow \sum_{X=1}^{6} d_{X}^{-1 / 2} A_{X} B_{X} C_{X}+d_{7}^{-1 / 2} \sum_{a, b, c=1,2} \sigma^{a b c} A_{7}^{a b} B_{7}^{b c} C_{7}^{c a}
$$

First of all, it is always the case that $\sigma^{111}=\sigma^{222}=1$. Now, it turns out that the two relevant sets of signs of the remaining $\sigma$ 's, appearing in the right formulas are either all equal to -1 :

$$
\text { variant a : } \quad \sigma^{112}=\sigma^{211}=\sigma^{121}=\sigma^{122}=\sigma^{212}=\sigma^{221}=-1
$$

or only two $\sigma^{a b c}$ equal to -1 . Depending on the order of fingers, it can be either $\sigma^{112}$ and $\sigma^{221}$ or two other pairs obtained by cyclic permutations from these. We will always choose the order in such a way that the first pattern is realized:

$$
\text { variant } b: \quad \sigma^{112}=\sigma^{221}=-1
$$

However, the choice between the two possibilities depends on the type of knot. The following ideal option with all signs positive

$$
\text { variant } c: \quad \sigma^{112}=\sigma^{211}=\sigma^{121}=\sigma^{122}=\sigma^{212}=\sigma^{221}=+1
$$

sometimes happens to provide right answers, but only when some of the pretzel lengths are unit and identities (6.6) relate (7.4) to (7.2)-(7.3).

It deserves mentioning that (7.3) explicitly breaks the cyclic symmetry between $A, B$ and $C$. Thus one can expect that it is relevant when such a symmetry is absent in 3 -finger 
configuration. That is., if we have two parallel and one antiparallel pretzel fingers. Having 3 parallel pretzel fingers is an impossible configuration to obtain knots. However, we can have three antiparallel pretzel fingers but the off-diagonal matrix elements in the second term of eq. (7.1) are vanishing.

\subsubsection{More fingers}

For 4-finger parallel pretzel knots the relevant choice is

$$
\sigma^{2211}=\sigma^{1122}=\sigma^{2112}=\sigma^{1221}=-1, \quad \text { all other } \quad \sigma^{a b c d}=+1
$$

For the 5-finger pretzel knot with one antiparallel finger (for the sake of definiteness, we take the first finger to be antiparallel pretzel ), the proper choice is

$$
\begin{aligned}
\sigma^{21111} & =\sigma^{22211}=\sigma^{22112}=\sigma^{21122}=\sigma^{21121}=\sigma^{21211}=\sigma^{12222}=\sigma^{11122}=\sigma^{11221}= \\
& =\sigma^{12211}=\sigma^{12212}=\sigma^{12122}=-1, \quad \text { all other } \quad \sigma^{a b c d}=+1
\end{aligned}
$$

Thus, we learn,

1. always $\sigma^{11 \ldots 1}=\sigma^{22 \ldots 2}=1$

2. there is a symmetry of simultaneous replacement of $1 \leftrightarrow 2$ in all indices.

3. Specifically for the parallel pretzel knots (which exist only for even number of fingers), there is also an additional symmetry: all sign factors obtained by a cyclic permutation are the same.

\subsection{Sign dependence on knots}

This list of relevant sign choices is not necessarily complete, but it is necessary and sufficient for all the examples that we studied so far. With these sign prescriptions, $H_{[21]}$ for knots are polynomials (otherwise denominators can occur) and coincide with the previously known answers from [37, 40, 41, 78].

Among knots belonging to starfish configurations, the 3 -finger configurations we checked are described by (7.3) (with the third finger chosen antiparallel but the first two fingers taken parallel) except knot $10_{71}$ where (7.2) choice is used. Similarly the 4 parallel finger knots and 5-finger (one antiparallel +4 parallel) configurations knots satisfy the rules described in section 7.1.2.

As for the double sum configurations, due to propagators connecting two $k$-valent vertices (see appendix), 10 152 is described by (7.3) in the both sums, $10_{153}$ is described by (7.3) in the sum over pretzel fingers and (7.2) in the second sum. The knot $10_{154}$ is described by (7.2) in the both sums.

Finally, the double fat configurations involving three summation variables (5.27) is described by (7.2) in the internal sum in (5.26) and (7.3) in the two sums within the ppS-blocks. 


\subsection{Explicit calculations in the pretzel case}

After the matrices $S, T$ and the sign factors $\sigma$ are explicitly known, it is straightforward to insert them into formulas of section 5 and obtain the colored HOMFLY polynomials in representation [21] for an enormous set of knots. In fact, the answers themselves are huge, thus it makes no sense to list them all, especially given the (large) number of examples. Therefore, we enumerate the classes of diagrams, and in the appendix we tabulate explicit examples. Here, for illustrative purposes, we present some general discussion of the pretzel case. A vast set of concrete examples can be found again in the appendix.

\subsubsection{Parallel}

The parallel pretzel finger is defined as

$$
\mathcal{A}_{1 X}^{\mathrm{par}}(n)=\left(S T^{n} S^{\dagger}\right)_{1 X}
$$

Unitarity of the $10 \times 10$ matrix $S$ implies that

$$
\mathcal{A}_{Y X}^{\mathrm{par}}(0)=\delta_{Y X}
$$

If instead of (7.7) we considered $\widetilde{\mathcal{A}^{\text {par }}}(n)_{1 X}=\left(S T^{n} \Lambda S^{\dagger}\right)_{1 X}=\left(S T^{n} S^{\dagger} \Lambda\right)_{1 X}$, where $\Lambda$ is the matrix of permutation of indices 9 and 10, this property would be broken: $\widetilde{\mathcal{A}^{\text {par }}}(0)_{Y X}=$ $\Lambda_{Y X}$, though the finger itself, at $Y=1$, would not be affected. This insertion of $\Lambda$ is just equivalent to the inverse order of indices of the right matrix $\bar{S}: T \Lambda \bar{S}=\sum_{X, c, d} T_{X}^{a b, c d} \bar{S}_{X Z}^{d c, e f}$ instead of $\sum_{X, c, d} T_{X}^{a b, c d} \bar{S}_{X Z}^{c d, e f}$.

Also vanishing are

$$
\mathcal{A}_{1,9}^{\mathrm{par}}( \pm 1)=\mathcal{A}_{1,10}^{\mathrm{par}}( \pm 1)=0
$$

but only for unit braid length, $n= \pm 1$.

The simple checks and comments are now in order.

For the 2-strand torus knots and links

$$
H_{[21]}^{[2, n]}=d_{[21]} \cdot \mathcal{A}_{11}^{\mathrm{par}}(-n)
$$

and for the 4-parallel-finger pretzel knots, like $6_{3}(2,-3,1,1), 7_{3}(4,1,1,1), 7_{5}(3,2,1,1)$ :

$$
H_{[21]}^{\mathrm{Pr}\left(n_{1}, n_{2}, n_{3}, n_{4}\right)}=\overline{\sum_{X}} \frac{d_{[21]}^{3}}{d_{X}} \mathcal{A}_{1 X}^{\mathrm{par}}\left(n_{1}\right) \mathcal{A}_{1 X}^{\mathrm{par}}\left(n_{2}\right) \mathcal{A}_{1 X}^{\mathrm{par}}\left(n_{3}\right) \mathcal{A}_{1 X}^{\mathrm{par}}\left(n_{4}\right)
$$

In the latter (4-finger) case the off-diagonal terms in (6.19) is non-vanishing, moreover, it is not a polynomial and cancels the non-polynomial part of the diagonal terms. For example, in the case of $6_{3}$ the off-diagonal contribution is

$$
\text { off - diagonal contribution to } 63: \quad-2\{q\}^{4} \cdot \frac{[6]}{[2]} \frac{D_{2} D_{-2}}{D_{0}} \cdot\left(\frac{[8]}{[2]} A q+A^{2} q^{4} D_{-4}\right)
$$

It is proportional to the fourth power of $\{q\}=q-q^{-1}$ and therefore vanishes in the limit $q=1$ (for special polynomials $[13,80]$ ). The same happens for knot $7_{5}$. However, for knot $7_{3}$, we observe that the off-diagonal contribution vanishes.

The simplest pair of parallel pretzel mutants is $\operatorname{Pr}(3,3,3,2)$ and $\operatorname{Pr}(3,3,2,3)$. We discuss this example, along with other mutants pairs of knots, in a separate section 8 . 


\subsubsection{Even antiparallel}

This finger depends on the even parameter $n$ and is given by the matrix element

$$
\mathcal{A}_{1 X}^{\mathrm{ea}}(\bar{n})=\left(\bar{S} \bar{T}^{n} \bar{S}^{\dagger}\right)_{1 X}
$$

Again, the unitarity $\mathcal{A}_{Y X}(\overline{0})=\delta_{Y X}$ makes this choice natural as compared with those with additional insertions of $\Lambda$.

For $n=0$, this matrix element is just $\delta_{X, 1}$.

For $n= \pm 2$, the matrix elements of $\mathcal{A}_{1 X}^{\text {ea }}$ with $X=9,10$ are very simple, similarly to the parallel case:

$$
\begin{aligned}
& X=9 \pm\{q\} \cdot \frac{[6] A^{ \pm 4}}{[2] D_{0}} \sqrt{\frac{D_{2} D_{-2}}{D_{1} D_{-1}}} \\
& X=10 \mp\{q\} \cdot \frac{[6] A^{ \pm 4}}{[2] D_{0}} \sqrt{\frac{D_{2} D_{-2}}{D_{1} D_{-1}}}
\end{aligned}
$$

The simplest check to be made is for the Hopf link, which can be represented both through parallel and antiparallel braid:

$$
\begin{aligned}
& H_{[21]}^{[2,2]}=\mathcal{A}_{11}^{\mathrm{par}}(2)=\mathcal{A}_{11}^{\mathrm{ea}}(-2)=\frac{1}{A^{9} q^{10}} \frac{1}{[3]\{q\}^{3}}\left(A^{6} q^{20}-2 A^{6} q^{18}-A^{4} q^{20}+3 A^{6} q^{16}+A^{4} q^{18}-\right. \\
& -4 A^{6} q^{14}-2 A^{4} q^{16}+5 A^{6} q^{12}+3 A^{4} q^{14}+A^{2} q^{16}-5 A^{6} q^{10}-4 A^{4} q^{12}+5 A^{6} q^{8}+3 A^{4} q^{10}+A^{2} q^{12}- \\
& \left.-4 A^{6} q^{6}-4 A^{4} q^{8}-A^{2} q^{10}+3 A^{6} q^{4}+3 A^{4} q^{6}+A^{2} q^{8}-q^{10}-2 A^{6} q^{2}-2 A^{4} q^{4}+A^{6}+A^{4} q^{2}+A^{2} q^{4}-A^{4}\right)
\end{aligned}
$$

This is the reduced HOMFLY invariant, and since it describes a link, it is not a polynomial.

Any combinations of even antiparallel fingers only provide links, not knots. The simplest knot family involving such fingers is $\operatorname{Pr}\left(n_{1}, n_{2}, \bar{m}\right)$, where two of the three fingers are parallel. Then

$$
H_{[21]}^{P r\left(n_{1}, n_{2}, \bar{m}\right)}=\overline{\sum_{X}} \frac{d_{[21]}^{2}}{\sqrt{d_{X}}} \mathcal{A}_{1 X}^{\mathrm{par}}\left(n_{1}\right) \mathcal{A}_{1 X}^{\mathrm{par}}\left(n_{2}\right) \mathcal{A}_{1 X}^{\mathrm{ea}}(\bar{m})
$$

This family includes knots $6_{2}(3,1, \overline{2}), \quad 8_{1}(1,1, \overline{6}), \quad 8_{2}(5,1, \overline{2}), \quad 8_{4}(3,1, \overline{4}), \quad 8_{5}(3,3, \overline{2})$, $8_{19}(3,3,-\overline{2}), \quad 8_{20}(3,-3, \overline{2}), \quad \ldots$ which are all 3 -strand and one can check that $(7.16)$ reproduces the known answers from [40].

Similarly, the five-finger family

$$
H_{[21]}^{P r\left(n_{1}, n_{2}, n_{3}, n_{4}, \bar{m}\right)}=\overline{\sum_{X}} \frac{d_{[21]}^{4}}{d_{X}^{3 / 2}} \mathcal{A}_{1 X}^{\mathrm{par}}\left(n_{1}\right) \mathcal{A}_{1 X}^{\mathrm{par}}\left(n_{2}\right) \mathcal{A}_{1 X}^{\mathrm{par}}\left(n_{3}\right) \mathcal{A}_{1 X}^{\mathrm{par}}\left(n_{4}\right) \mathcal{A}_{1 X}^{\mathrm{ea}}(\bar{m})
$$

contains $7_{6}(1,1,-3,1, \overline{2}), 8_{3}(1,1,1,1, \overline{4}), 8_{6}(1,1,1,3, \overline{2}), \ldots$ At least for these three knots (7.17), we get polynomials and they satisfy the consistency checks putforth in section 7.4.

\subsubsection{Odd antiparallel}

This finger depends on the odd parameter $n$ and is given by the matrix element

$$
\mathcal{A}_{1 X}^{\mathrm{oa}}(\bar{n})=\left(\bar{S}^{\dagger} \bar{T}^{n} S\right)_{1 X}
$$


This time $n$ is odd and cannot vanish, therefore there is no obstacle for inserting $\Lambda$ and writing $\left(\bar{S}^{\dagger} T^{n} \Lambda S\right)_{1 X}$ instead. However, this does not actually affect the answers, at least in the cases discussed here.

Elementary calculation or application of identity (6.4) gives that at $n= \pm 1$

$$
\mathcal{A}_{1 X}^{\mathrm{oa}}( \pm 1)=\left\{\begin{array}{cc}
\frac{\sqrt{d_{X}}}{d_{[21]}} \cdot T_{X X}^{\mp 1} & X=1, \ldots, 8 \\
0 & X=9,10
\end{array}\right.
$$

where $X$ belongs to the parallel sector, $X \in R \otimes R$. Once again, this immediately provides the answers for the trefoil:

$$
\sum_{X \in[21] \otimes[21]} \frac{d_{[21]}^{2}}{\sqrt{d_{X}}} \cdot\left(\mathcal{A}_{1 X}^{\text {oa }}(1)\right)^{3}=\frac{1}{d_{[21]}} \sum_{X \in[21] \otimes[21]} d_{X}^{2} T_{X X}^{-3}
$$

and for the unknot

$$
\sum_{X \in[21] \otimes[21]} \frac{d_{[21]}^{2}}{\sqrt{d_{X}}} \cdot \mathcal{A}_{1 X}^{\mathrm{oa}}(-1)\left(\mathcal{A}_{1 X}^{\mathrm{oa}}(1)\right)^{2}=\frac{1}{d_{[21]}} \sum_{X \in[21] \otimes[21]} d_{X}^{2} T_{X X}^{-1}=1
$$

For other values of $n$ these matrix elements are multiplied by polynomials:

$$
\mathcal{A}_{1 X}^{\mathrm{oa}}( \pm \bar{n})=\mathcal{A}_{1 X}^{\mathrm{oa}}( \pm \overline{1}) \cdot \mathcal{P}_{X}^{\mathrm{oa}}\left(n \mid A^{\mp 1}, q^{\mp 1}\right)
$$

In particular, this means that the odd antiparallel fingers vanish at $X=9,10$ for all values $n$, which means that odd antiparallel pretzel mutants are not distinguished by the colored HOMFLY polynomials in representation [21].

The polynomials $\mathcal{P}$ themselves are, however, somewhat involved. For instance,

$$
\begin{aligned}
& \mathcal{P}_{1}(3)=\frac{1}{q^{10}}\left(-A^{4} q^{16}+A^{4} q^{14}+A^{6} q^{10}-A^{2} q^{14}+2 A^{4} q^{10}+2 A^{2} q^{12}-A^{4} q^{8}-\right. \\
& \left.-A^{2} q^{10}-q^{12}+2 A^{2} q^{8}+2 q^{10}-A^{4} q^{4}-3 A^{2} q^{6}-q^{8}+A^{2} q^{4}+q^{6}-A^{2} q^{2}-2 q^{4}+A^{2}+q^{2}\right)
\end{aligned}
$$

The twist knots are pretzel $T w_{k}=\operatorname{Pr}(\overline{1}, \overline{1}, \overline{2 k-1})$ i.e. are made from three odd antiparallel fingers:

$$
H_{[21]}^{P r\left(\bar{n}_{1}, \bar{n}_{2}, \bar{n}_{3}\right)}=\sum_{X=1}^{8} \frac{d_{[21]}^{2}}{\sqrt{d_{X}}} \mathcal{A}_{1 X}^{\mathrm{oa}}\left(\bar{n}_{1}\right) \mathcal{A}_{1 X}^{\mathrm{oa}}\left(\bar{n}_{2}\right) \mathcal{A}_{1 X}^{\mathrm{oa}}\left(\bar{n}_{3}\right)
$$

The off-diagonal terms in (6.19) vanish for odd antiparallel fingers implying that there is no difference between $\sum_{X=1}^{8}$ and $\overline{\sum_{X}}$. The answers for twist knots agree with those in refs. [40, 41, 78]. Other members of the same three-finger family are: $7_{4}(\overline{3}, \overline{3}, \overline{1})$, $9_{5}(-\overline{1},-\overline{3},-\overline{5}), \quad 9_{35}(\overline{3}, \overline{3}, \overline{3}), \quad 9_{46} \quad(\overline{3},-\overline{3}, \overline{3}), \quad 10_{3}(\overline{1}, \overline{5},-\overline{5}), \ldots$

\subsection{Checks}

All answers for the HOMFLY polynomials in representation [21] in this paper for the knots that have a three braid representation were compared with the results obtained by the cabling method of $[37,40]$. Besides, there are five types of self-consistency checks one can putforth to test our answers. The first four are true for all our examples, the fifth one is more tedious and was only partly verified. 


\subsubsection{Special polynomials}

As conjectured in $[13,80]$ and proved in [81], the "special" polynomials, i.e. reduced HOMFLY polynomials at $q=1$, obey the factorization rule

$$
H_{R}^{\mathcal{K}}(q=1, A)=\left(H_{\square}^{\mathcal{K}}(q=1, A)\right)^{|R|}
$$

i.e. are unambiguously restored from those in the fundamental representation $R=[1]=\square$. The size $|R|$ of the Young diagram $R$ is just the number of boxes, $|[21]|=3$.

For superpolynomials this factorization gets far more interesting, see [14, 38, 82, 83] and [37].

\subsubsection{Alexander polynomials for 1-hook representations}

As conjectured in [80] a "dual" factorization property holds at $A=1$, i.e. for the Alexander polynomials, but only for representations $R$ described by single-hook diagrams:

$$
H_{R}^{\mathcal{K}}(A=1, q)=H_{\square}^{\mathcal{K}}\left(A=1, q^{|R|}\right)
$$

Representation [21] belongs to this class.

Neither any meaning, nor generalizations of this remarkable factorization property is currently available.

\subsubsection{Jones polynomials}

For $S U_{q}(2)$, i.e. for $A=q^{2}$ all two-line Young diagrams $\left[r_{1} r_{2}\right]$ get equivalent to the singleline $\left[r_{1}-r_{2}\right]$, in particular, [21] gets indistinguishable from [1]. This means that

$$
H_{[21]}^{\mathcal{K}}\left(A=q^{2}\right)=H_{\square}^{\mathcal{K}}\left(A=q^{2}\right)=\text { Jones }^{\mathcal{K}}
$$

\subsubsection{The weak form of differential expansion}

Expressions for colored knot polynomials are extremely complicated, but in fact they have a lot of hidden structure and satisfy a lot of non-trivial relations. Understanding of these structures nicknamed "differential expansions" [39, 70, 80] because their first traces were observed in [11] devoted to the study of Khovanov-Rozansky "differentials" is still very poor. In its weakest possible form, the differential expansion conjecture implies for representation [21] that $[37,78]$

$H_{[21]}^{\mathcal{K}}(A, q)=1+F_{1}^{\mathcal{K}}\left(A^{2}, q^{2}\right)\left(\left\{A q^{3}\right\}\left\{A / q^{3}\right\}+\left\{A q^{2}\right\}\{A\}+\{A\}\left\{A / q^{2}\right\}\right)+\left\{A q^{2}\right\}\left\{A / q^{2}\right\} \cdot G_{[21]}^{\mathcal{K}}\left(A^{2}, q^{2}\right)$

with some (complicated) function $G_{[21]}^{\mathcal{K}}$, where $F_{1}^{\mathcal{K}}\left(A^{2}, q^{2}\right)=F_{1}^{\mathcal{K}}\left(A^{2}, q^{-2}\right)$ is the coefficient in

$$
H_{\square}^{\mathcal{K}}(A, q)=1+F_{1}^{\mathcal{K}}\left(A^{2}, q^{2}\right)\{A q\}\{A / q\}
$$

and $H_{R}$ are the reduced HOMFLY polynomials. In other words certain linear combination of $H_{[21]}$ and $H_{[1]}$ should vanish at $A=q^{ \pm 2}$, i.e. in the case of $S U_{q}(2)$. 


\subsubsection{Quasiclassical expansion and Vassiliev invariants}

Differential expansions from the previous paragraph are examples of cleverly-structured quasiclassical expansions in parameters like $\hbar$, where $q=e^{\hbar}$ and $A=q^{N \hbar}$ or $z=\{q\}=$ $q-1 / q$. The most structured expansion of this type, known so far is the Hurwitz-style formula [84-86] for reduced colored HOMFLY:

$$
H_{R}^{\mathcal{K}}\left(q=e^{\hbar}, A\right)=\left(H_{\square}(q=1, A)\right)^{|R|} \cdot \exp \left(\sum_{\Delta} \hbar^{|\Delta|+l(\Delta)-2+k} \Sigma_{\Delta, k}^{\mathcal{K}}(A) \varphi_{R}(\Delta)\right)
$$

Here $\varphi_{R}(\Delta)$ are characters of the universal symmetric group (defined as in $\left.[72,73]\right), l(\Delta)$ is the number of lines in the Young diagram $\Delta$ and the coefficients of generalized special polynomials $\Sigma_{\Delta, k}^{\mathcal{K}}(A)$ are made from the Vassiliev invariants.

This formula includes (7.25) as a particular case and is also closely related to (7.28). It imposes non-trivial restrictions on the coefficients of colored polynomials. As already mentioned, we made only a few simple checks of our answers with this formula, and it is very interesting to extend them in order to understand what are the really independent "degrees of freedom" in $H_{[21]}$, as compared to those already captured by the colored HOMFLY polynomials in symmetric representations. This study can be also helpful for superpolynomial extensions of the [21]-colored knot polynomials, which still remains a complete mystery.

\section{Mutants}

Of special interest among the newly available answers are those for the pairs of mutants, i.e. knots related by the mutation transformation, which are inseparable by knot polynomials in symmetric representations.

\subsection{Generalities}

Mutation in knot theory is the transformation of link diagram, when one cuts a sub-diagram with exactly four external legs, rotate and glue it back to the original position. Within the Reshetikhin-Turaev approach, it is clear that cutting corresponds to decomposition of knot polynomial in the channel $R_{1} \otimes R_{2}$ and mutation is a rotation in the spaces of intertwining operators $W_{R_{1} R_{2}}^{Q}: R_{1} \otimes R_{2} \rightarrow Q$. If these spaces are one-dimensional, like in the case of rectangular representations $R_{1}=R_{2}$ or $\bar{R}_{1}=R_{2}$, the mutation does not affect the corresponding HOMFLY polynomial. Thus, mutants can be distinguished by colored HOMFLY polynomials in non-rectangular representations, the first of which is $R=[21]$. The difference

$$
\begin{gathered}
H_{[21]}^{\text {mutant }_{1}}-H_{[21]}^{\text {mutant }_{2}}=\{q\}^{11} \cdot D_{3}^{2} D_{2} D_{0} D_{-2} D_{-3}^{2} \cdot A^{\gamma} \cdot M_{[21]}^{\text {mutant }}\left(q^{2}\right)= \\
=\{q\}^{4} \cdot\left\{A q^{3}\right\}^{2}\left\{A q^{2}\right\}\{A\}\left\{A / q^{2}\right\}\left\{A / q^{3}\right\}^{2} \cdot A^{\gamma} \cdot M_{[21]}^{\text {mutant }}\left(q^{2}\right)
\end{gathered}
$$

has the universal prefactor. The differentials $D_{ \pm 2}$ and $D_{0}$ appear in it because the Alexander and the Jones polynomials at $A=1$ and $A=q^{2}$ are not affected by the mutation and, thus, the difference should vanish for $S U_{q}(N)$ with $N=0$ and $N=2$. The Young diagram is symmetric under row-column interchange (also called rank-level duality $[13,15,80])$. Hence the polynomial in variables $A$ and $q$ must remain same when we change $q \rightarrow q^{-1}$ and, hence, the difference should vanish also at $A=q^{-2}$. Vanishing for 
$S U_{q}(3)$ follows from a more involved argument of ref. [74]. There are no factors $D_{1} D_{-1}$, because we consider the reduced HOMFLY polynomial, which is equal to the original unreduced one divided by $d_{21}=\frac{D_{1} D_{0} D_{-1}}{[3]}$. The additional $A$-independent factor $\{q\}^{4}$ seems to be typical for all non-diagonal terms considered in this paper, and this provides an explanation for power 11 in (8.1). In all examples, which we managed to analyze, $M^{\text {mutant }}\left(q^{2}\right)$ is, indeed, a function of $q$ only, with no $A$-dependence.

\subsection{Examples of mutants}

The mutant pairs appear for the first time for knots with eleven intersections, where there are 16 pairs (n\&a in the knots denote alternating or non-alternating):

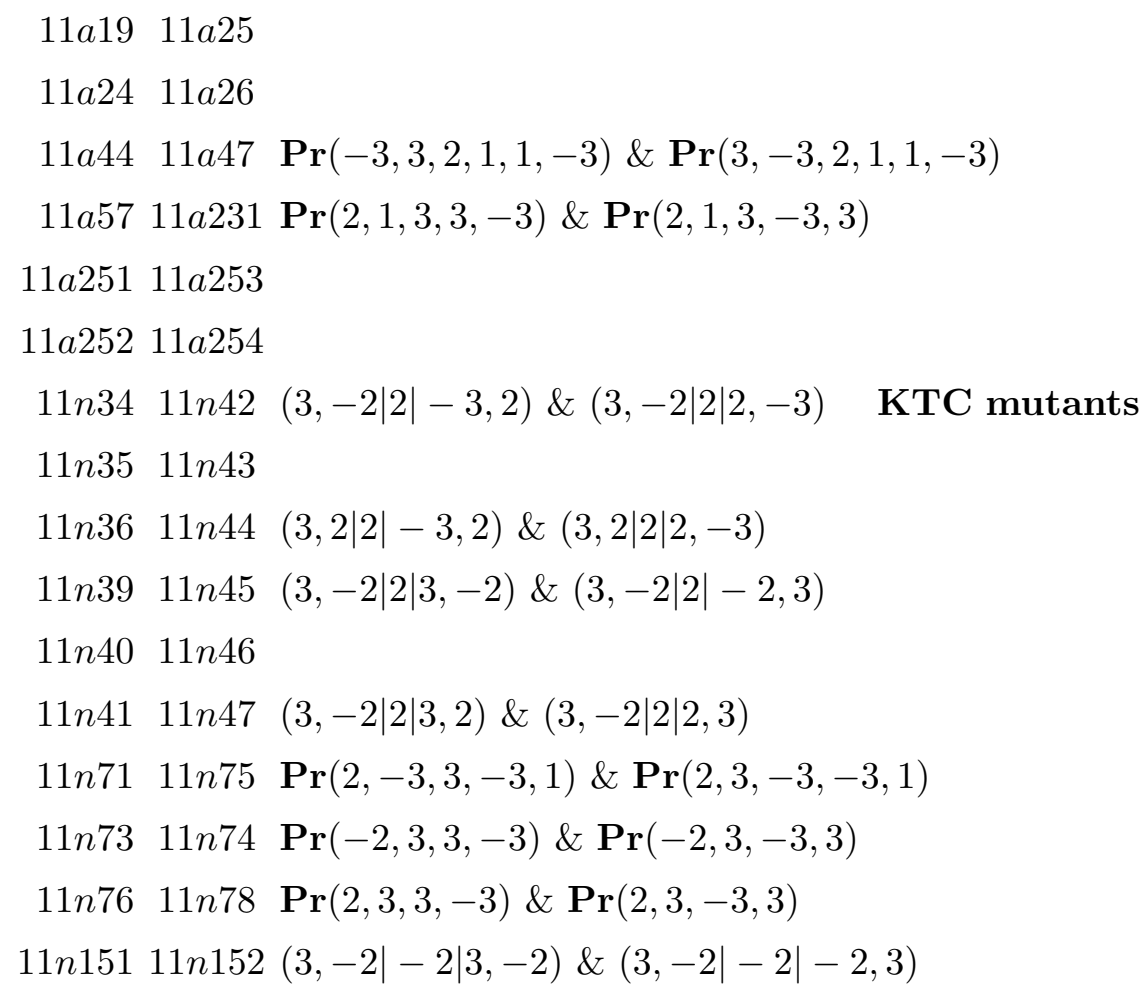

Of these, the KTC pair is most famous, probably because their Alexander polynomials are just unity. The simplest mutants are the pretzel ones: there are five such pairs in the above list, see also eq. (8.4) below. For enumeration of mutants with up to 16 intersections see [87-89].

\subsection{HOMFLY for the Pretzel mutants}

First of all, we repeat that $H_{[21]}$ do not distinguish pretzel mutants made from antiparallel pretzel fingers of odd lengths, see section 7.3 .3 above. The simplest example of this kind are 12-intersection pair of mutant links $\operatorname{Pr}(\overline{3}, \overline{3},-\overline{3},-\overline{3}) \& \operatorname{Pr}(\overline{3},-\overline{3}, \overline{3},-\overline{3})$ and 15-intersection pair of mutant knots $\operatorname{Pr}(\overline{3}, \overline{3}, \overline{3},-\overline{3},-\overline{3}) \& \operatorname{Pr}(\overline{3}, \overline{3},-\overline{3}, \overline{3},-\overline{3})$. In both cases the links/knots in the pair seem topologically different, but

$$
H_{[21]}^{\operatorname{Pr}(\overline{3}, \overline{3}, \overline{3},-\overline{3},-\overline{3})}-H_{[21]}^{\operatorname{Pr}(\overline{3}, \overline{3},-\overline{3}, \overline{3},-\overline{3})}=0
$$

and so on. 
For the pretzel parallel fingers the situation is better. The simplest parallel finger pretzel mutants are made from four fingers of lengths $3,3,3,2$ and therefore contain 11 intersections. Of eight potential mutant pairs in this set there are actually only two pairs with distinct polynomials. In fact, pretzel knot $\operatorname{Pr}(3,3,3, \pm 2)=\operatorname{Pr}(3,3, \pm 2,3)$ because of the cyclic symmetry, $\operatorname{Pr}(3,3,-3, \pm 2 \mid A, q)=\operatorname{Pr}\left(3,-3,-3, \mp 2 \mid A^{-1}, q\right)$, and, finally, $\operatorname{Pr}(3,-3,-3, \pm 2 \mid A, q)=\operatorname{Pr}\left(3,3,-3, \mp 2 \mid A^{-1}, q^{-1}\right)$ if one reads the sequence of lengths in the opposite direction and applies the cyclic symmetry. This leaves two pairs of 11-intersection mutants, which are pretzels with four parallel fingers: $11 n 73 \& 11 n 74$ $(\operatorname{Pr}(-2,3,3,-3) \& \operatorname{Pr}(-2,3,-3,3))$ and $11 n 76 \& 11 n 78(\operatorname{Pr}(2,3,3,-3) \& \operatorname{Pr}(2,3,-3,3))$. For them

$$
\begin{gathered}
H_{[21]}^{P r(-2,3,-3,3)}-H_{[21]}^{P r(-2,3,3,-3)}=A^{-3} \cdot \frac{[3]^{2}[8][14]}{[2]^{2}[7]} \cdot\{q\}^{4}\left\{A q^{3}\right\}^{3}\left\{A q^{2}\right\}\{A\}\left\{A / q^{2}\right\}\left\{A / q^{3}\right\}^{2}, \\
H_{[21]}^{P r(2,3,3,-3)}-H_{[21]}^{P r(2,3,-3,3)}=A^{-15} \cdot \frac{[3]^{2}[8]}{[2]} \cdot\{q\}^{4}\left\{A q^{3}\right\}^{3}\left\{A q^{2}\right\}\{A\}\left\{A / q^{2}\right\}\left\{A / q^{3}\right\}^{2}(8.4)
\end{gathered}
$$

One can easily analyze any other mutant pair of this kind, for example:

$$
H_{[21]}^{P r(9,3,2,5)}-H_{[21]}^{P r(9,3,5,2)}=-A^{-57} \cdot \frac{[6]^{2}[8][10][11]}{[2]^{3}[4]}\left(\frac{[14]}{[2]}-\frac{[12]}{[6]}\right) \cdot\{q\}^{4}\left\{A q^{3}\right\}^{3}\left\{A q^{2}\right\}\{A\}\left\{A / q^{2}\right\}\left\{A / q^{3}\right\}^{2}
$$

In fact, the pretzel mutant pairs provide us with a much better set of examples than concrete knots like the KTC pair: one can easily construct whole families of mutants. An example of such a family we consider in the next section.

Note that among the pretzel mutant pairs there are more knots with 11 intersections, though their pretzel presentation has more intersections (12 and even 13). These are $11 n 71 \& 11 n 75(\operatorname{Pr}(2,-3,3,-3,1) \& \operatorname{Pr}(2,3,-3,-3,1))$, $11 a 44 \& 11 a 47(\operatorname{Pr}(-3,3,2,1,1,-3) \& \operatorname{Pr}(3,-3,2,1,1,-3))$ and $11 a 57 \& 11 a 231$ $(\operatorname{Pr}(2,1,3,3,-3) \& \operatorname{Pr}(2,1,3,-3,3))$. The differences of their HOMFLY are:

$$
\begin{gathered}
H_{[21]}^{P r(2,-3,3,-3,1)}-H_{[21]}^{P r(2,3,-3,-3,1)}=A^{13} \frac{[3]^{2}[8]}{[2]} \cdot\{q\}^{4}\left\{A q^{3}\right\}^{3}\left\{A q^{2}\right\}\{A\}\left\{A / q^{2}\right\}\left\{A / q^{3}\right\}^{2}(8.6) \\
H_{[21]}^{P r(2,1,3,-3,3)}-H_{[21]}^{P r(2,1,3,3,-3)}=A^{-5} \frac{[3]^{2}[8][14]}{[2]^{2}[7]} \cdot\{q\}^{4}\left\{A q^{3}\right\}^{3}\left\{A q^{2}\right\}\{A\}\left\{A / q^{2}\right\}\left\{A / q^{3}\right\}^{2} \\
H_{[21]}^{\operatorname{Pr}(-3,3,2,1,1,-3)}-H_{[21]}^{\operatorname{Pr}(3,-3,2,1,1,-3)}=A \frac{[3]^{2}[8][14]}{[2]^{2}[7]} \cdot\{q\}^{4}\left\{A q^{3}\right\}^{3}\left\{A q^{2}\right\}\{A\}\left\{A / q^{2}\right\}\left\{A / q^{3}\right\}^{2}
\end{gathered}
$$

\subsection{KTC mutants}

The Kinoshita-Terasaka (11n42) and Conway (11n34) knots (KTC mutants) are respectively $M(3,-2|2|-3,2)$ and $M(3-2|2| 2,-3)$. Both knots have representations with 11intersection, but for our purposes the realization with 12 intersections is more convenient, see figure 3 .

These diagrams are already easy to bring to the form (5.26) with $(p, q, r, s)=$ $(3,-2,-3,2)$ and $(3,-2,2,-3)$ for $11 n 42$ and $11 n 34$ respectively. Their HOMFLY are: ${ }^{2}$

\footnotetext{
${ }^{2}$ We use here the notation from $[43,44]$. Matrix lists the coefficients of a polynomial in $A^{2}$ and $q^{2}$ by the following rule:

$$
q^{10} A^{16}\left(\begin{array}{ll}
3 & 4 \\
1 & 2
\end{array}\right)=q^{10} A^{16}+2 q^{12} A^{16}+3 q^{10} A^{18}+4 q^{12} A^{18} .
$$




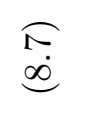

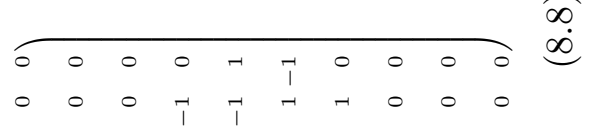

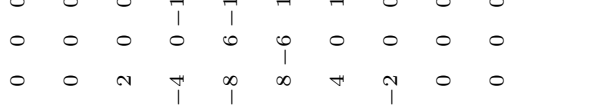

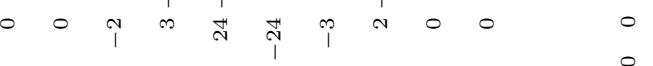

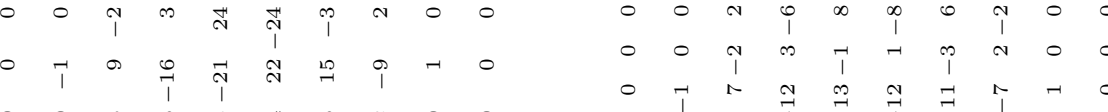

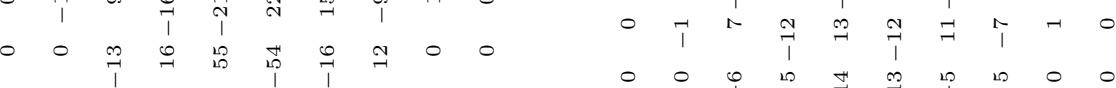$$
\text { - } \begin{aligned}
& 0 \\
& 0
\end{aligned}
$$

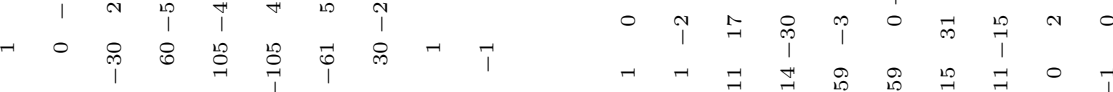

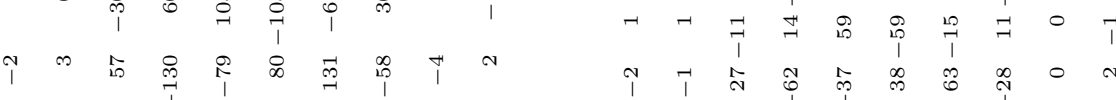$$
\text { 以 }
$$

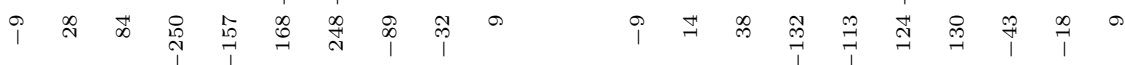

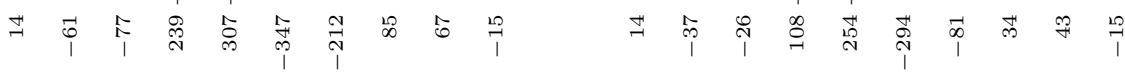

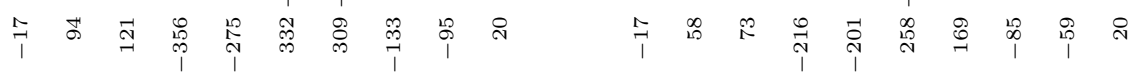

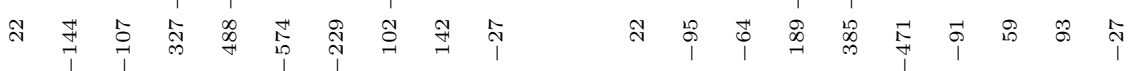

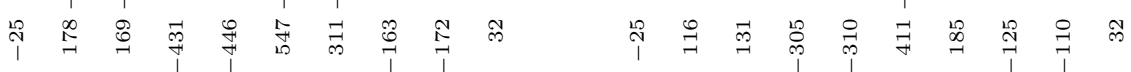

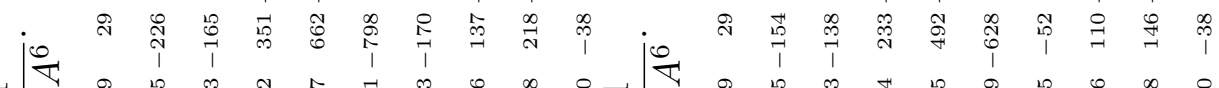

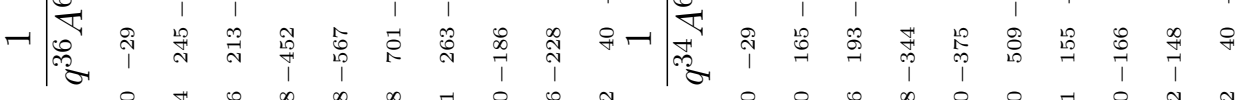
川

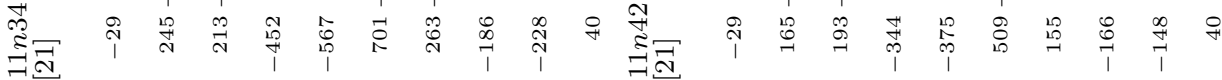

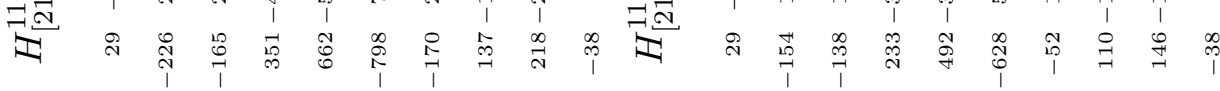

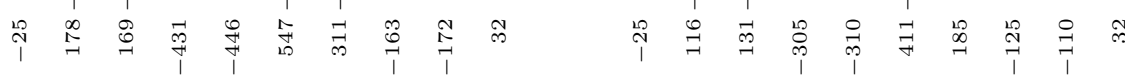

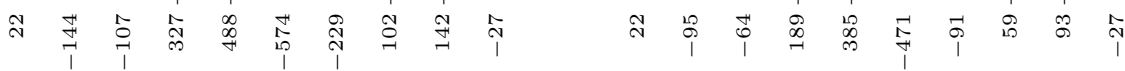

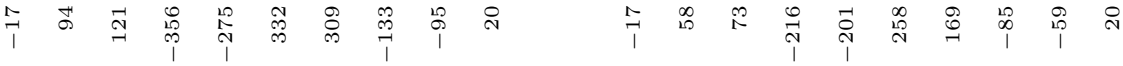

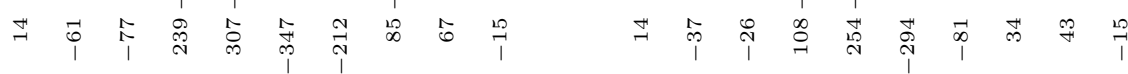

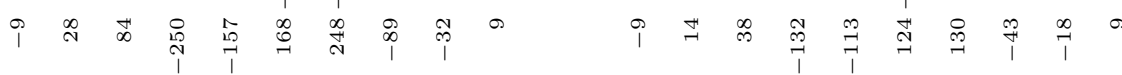

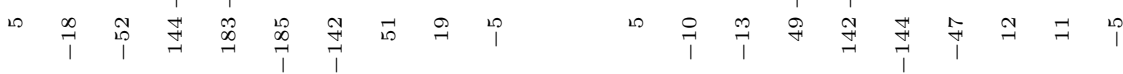
彳

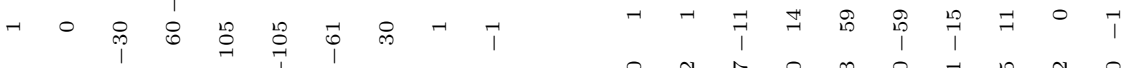

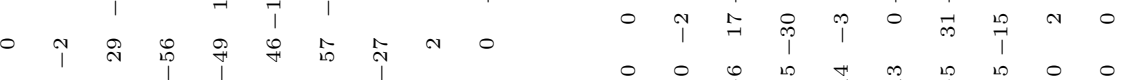

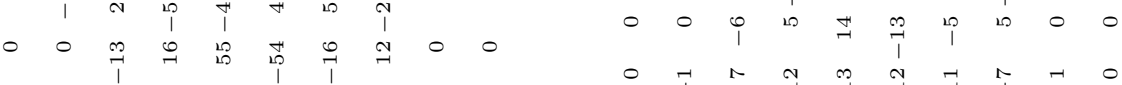

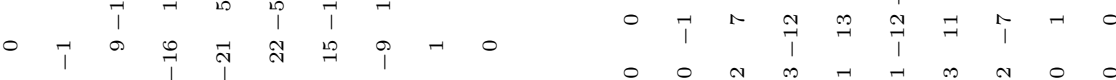
- 0 - $\begin{gathered}0 \\ 0\end{gathered}$

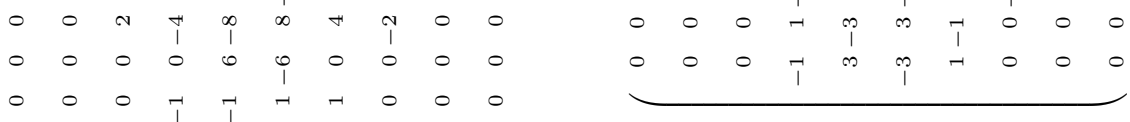

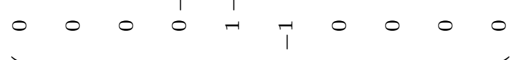




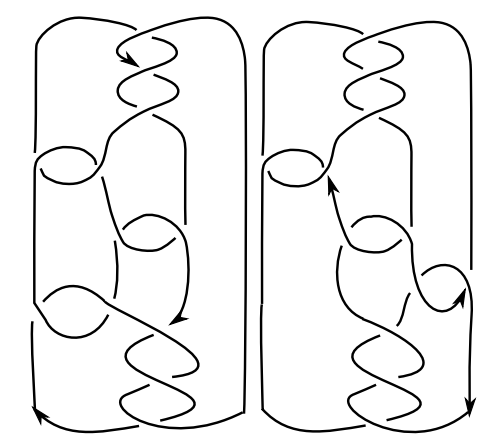

Figure 3. (a) Kinoshita-Terasaka (b) Conway.

The KTC knots can be viewed as two 3-fingers connected by propagator involving horizontal braid with $m=2$ crossings. Changing $m=2 \longrightarrow m=-2$ simply switches between these two mutant polynomials.

At $q=1$ both these polynomials are cubes of the special polynomial,

$$
H_{[21]}(q=1)=\left(H_{[1]}(q=1)\right)^{3}=\left(\frac{2 A^{6}-6 A^{4}+7 A^{2}-2}{A^{2}}\right)^{3}
$$

At $A=q^{2}$ both reproduce the same Jones

$$
H_{[21]}\left(A=q^{2}\right)=H_{[1]}\left(A=q^{2}\right)=q^{12}-2 q^{10}+2 q^{8}-2 q^{6}+q^{4}+2 q^{-2}-2 q^{-4}+2 q^{-6}-q^{-8}
$$

and at $A=1$ the Alexander polynomial, which in this particular case is just unity,

$$
H_{[21]}(A=1, q)=H_{[1]}\left(A=1, q^{3}\right)=1
$$

The first terms of the differential expansion (see section 7.4) in the classical limit, $A=q^{N}$, $q=e^{\hbar}$, are

$$
H_{[21]}=1+2\{A\}\left(\left\{A q^{3}\right\}\left\{A / q^{3}\right\}+\left\{A q^{2}\right\}\{A\}+\{A\}\left\{A / q^{2}\right\}\right)+O\left(\hbar^{4}\right)
$$

In accordance with [74], the difference between the two polynomials shows up in the order $\hbar^{11}$, which is related to the power 11 of $\{q\}$ in (8.1).

The complete difference is

$$
\begin{gathered}
H_{[21]}^{M(3,-2|2|-3,2)}-H_{[21]}^{M(3,-2|2| 2,-3)}=-\rho^{2} A^{3} \cdot D_{3}^{2} D_{2} D_{0} D_{-2} D_{-3}^{2} \cdot z^{11} \\
=-\rho^{2} A^{3} \cdot\{q\}^{4}\left\{A q^{3}\right\}^{2}\left\{A q^{2}\right\}\{A\}\left\{A / q^{2}\right\}\left\{A / q^{3}\right\}^{2}
\end{gathered}
$$

with $\rho=\frac{[3][14]}{[2][7]}$ and $z=q-q^{-1}=\{q\}$. It perfectly matches the result of [75]. The difference could actually be calculated for three-cabled knots (because symmetric and antisymmetric representations [3] and [111] do not contribute to it), what allows one to make calculations with the help of the ordinary skein relations. However, the answers for individual $H_{[21]}$ could not be obtained in that way. 


\subsection{Other mutant pairs}

In the list (8.2) of 11-intersection mutant pairs there are four more pairs, for which the fat tree description is already provided. In fact, from this point of view they belong exactly to the same class as the KTC mutants, just some intersection signs are different. The differences are

$$
\begin{gathered}
H_{[21]}^{11 n 36}-H_{[21]}^{11 n 44}=A^{-9} \frac{[3]^{2}[14]}{[2][7]} \cdot\{q\}^{4}\left\{A q^{3}\right\}^{3}\left\{A q^{2}\right\}\{A\}\left\{A / q^{2}\right\}\left\{A / q^{3}\right\}^{2} \\
H_{[21]}^{11 n 39}-H_{[21]}^{11 n 45}=A^{-3} \frac{[3]^{2}[14]^{2}}{[2]^{2}[7]^{2}} \cdot\{q\}^{4}\left\{A q^{3}\right\}^{3}\left\{A q^{2}\right\}\{A\}\left\{A / q^{2}\right\}\left\{A / q^{3}\right\}^{2} \\
H_{[21]}^{11 n 41}-H_{[21]}^{11 n 47}=A^{-15} \frac{[3]^{2}[14]}{[2][7]} \cdot\{q\}^{4}\left\{A q^{3}\right\}^{3}\left\{A q^{2}\right\}\{A\}\left\{A / q^{2}\right\}\left\{A / q^{3}\right\}^{2} \\
H_{[21]}^{11 n 151}-H_{[21]}^{11 n 152}=A^{-9} \frac{[3]^{2}[14]^{2}}{[2]^{2}[7]^{2}} \cdot\{q\}^{4}\left\{A q^{3}\right\}^{3}\left\{A q^{2}\right\}\{A\}\left\{A / q^{2}\right\}\left\{A / q^{3}\right\}^{2}
\end{gathered}
$$

Somewhat surprisingly, some differences are the same for different pairs: for $11 \mathrm{n} 36 / 11 \mathrm{n} 44$ and $11 \mathrm{n} 41 / 11 \mathrm{n} 47$, as well as for $11 \mathrm{n} 39 / 11 \mathrm{n} 45$ and $11 \mathrm{n} 151 / 11 \mathrm{n} 152$. The same phenomenon one could observe for the pretzel mutants: the differences are the same for pairs: 11n71/11n75 and 11n76/11n78; 11a47/11a44, 11a57/11a231 and 11n73/11n74 (in the latter case the three differences coincide), see section 8.3. The entire HOMFLY are, of course quite different, see the appendix.

It remains to be seen, if the remaining pairs in (8.2) possess a double fat tree description.

\section{Evolution method in application to mutant families}

Of course, most interesting are not just particular knots, but entire families, depending on various parameters. The way to study this kind of problems is provided by the evolution method of [13, 70]. For its application to knot polynomials in representation [21] see [78], but there only the simplest family of twist knots was considered (the torus knots were described in this way in arbitrary representation $[12-14,76,77]$, but this is a much simpler exercise, because of the clear algebraic nature of the torus family). We briefly remind this story and then extend consideration to the first mutant-containing family.

\subsection{Twist knots $[40,41,78]$}

The [21]-colored HOMFLY for generic twist knots was obtained by the evolution method in [78]:

$$
\begin{aligned}
H_{[21]}^{(k)} & =u_{3} A^{6 k}+\left(u_{2 p} q^{4 k}+u_{20}+u_{2 m} q^{-4 k}\right) A^{4 k}+u_{1} A^{2 k}+u_{0} \\
u_{3} & =-A^{3} \frac{\left\{A q^{3}\right\}\left\{A / q^{3}\right\}\{A q\}\{A / q\}}{\{A\}} \\
u_{2 p} & =\frac{[3]}{[2]^{2}} A^{3} \frac{\left\{A q^{3}\right\}\{A\}}{\{A q\}}\left([2] A^{2} q^{-3}-\left(q^{4}+1-q^{-2}+q^{-4}\right)\right)
\end{aligned}
$$




$$
\begin{aligned}
u_{2} & =-2\{q\}^{2} A^{3}\left(\frac{[3]}{[2]}\right)^{2} \frac{\left\{A q^{2}\right\}\left\{A / q^{2}\right\}}{\{A\}} \\
u_{2 m} & =\frac{[3]}{[2]^{2}} A^{3} \frac{\left\{A q^{-3}\right\}\{A\}}{\left\{A q^{-1}\right\}}\left([2] A^{2} q^{3}-\left(q^{4}+1-q^{2}+q^{-4}\right)\right) \\
u_{1} & =-\frac{[3] A^{3}}{\{A\}}\left(A^{4}-\left(q^{6}+q^{-6}\right) A^{2}+\left(2 q^{6}-4 q^{4}+4 q^{2}-3+4 q^{-2}-4 q^{-4}+2 q^{-6}\right)\right) \\
u_{0} & =\frac{A^{3}}{\{A q\}\{A\}\{A / q\}}\left(A^{6}-\frac{[3][10]}{[2][5]} A^{4}+\frac{[3][10]^{2}}{[2]^{2}[5]^{2}} A^{2}-\frac{[10][14]}{[2]^{2}[5][7]}\right)
\end{aligned}
$$

This example is already quite interesting for the study of differential expansion [39] beyond (anti)symmetric representations. However, this family does not contain mutant pairs and, hence we need to look for knots beyond this family.

\subsection{4-finger pretzel knots}

The simplest multi-parametric family containing mutant pairs is the four-parallel-finger pretzels $\operatorname{Pr}\left(n_{1}, n_{2}, n_{3}, n_{4}\right)$ with even $n_{1}$ and $n_{2}, n_{3}, n_{4}$ odd. In this case, one has

$$
\frac{H_{[21]}^{\operatorname{Pr}\left(n_{1}, n_{2}, n_{3}, n_{4}\right)}-H_{[21]}^{\operatorname{Pr}\left(n_{1}, n_{2}, n_{4}, n_{3}\right)}}{A^{-3\left(n_{1}+n_{2}+n_{3}+n_{4}\right)}\{q\}^{4}\{A q\}^{3}\left\{A q^{2}\right\}\{A\}\left\{A / q^{2}\right\}\left\{A / q^{3}\right\}}=\frac{1}{\{q\}^{11}} \cdot F\left(n_{1} \mid n_{2}\right) \cdot G\left(n_{3}, n_{4}\right)
$$

with

$$
\begin{aligned}
& G\left(n_{3}, n_{4}\right)=\left(q^{4\left(n_{3}+n_{4}\right)}+q^{-4\left(n_{3}+n_{4}\right)}\right)\left(q^{n_{4}-n_{3}}-q^{n_{3}-n_{4}}\right) \\
& +\frac{[4]^{2}}{[2]^{2}}\left(q^{n+3+n_{4}}+q^{-n_{3}-n_{4}}\right)\left(q^{4\left(n_{4}-n_{3}\right)}-q^{4\left(n_{3}-n_{4}\right)}\right)+ \\
& +\frac{[3][6]}{[2][5]}\left(q^{5\left(n_{3}-n_{4}\right)}-q^{5\left(n_{4}-n_{3}\right)}\right)+\frac{[5]}{[3]}\left(q^{4}+3+q^{-4}\right)\left(q^{3\left(n_{3}-n_{4}\right)}-q^{3\left(n_{4}-n_{3}\right)}\right)+ \\
& +\frac{[2][4][6]}{[3][5]}\left(q^{5 n_{3}}-q^{-5 n_{3}}-q^{5 n_{4}}+q^{-5 n_{4}}\right)+\frac{[2][4][6]}{[3]^{2}}\left(-q^{3 n_{3}}+q^{-3 n_{3}}+q^{3 n_{4}}-q^{-3 n_{4}}\right)
\end{aligned}
$$

and

$$
F\left(n_{1}=2 \mid n_{2}\right)=\{q\}^{2} \frac{[3]}{[2]^{4}[4]^{3}[5]^{2}[6]^{2}}\left([6]\left[5 n_{2}-3\right]-[2][5]\left[3 n_{2}-5\right]-\frac{[2]^{2}[4][6]}{[3]}+\{q\}^{2}[2][5]\left[3 n_{2}-1\right]\right)
$$

For generic even values of $n_{1}$ expression is more complicated:

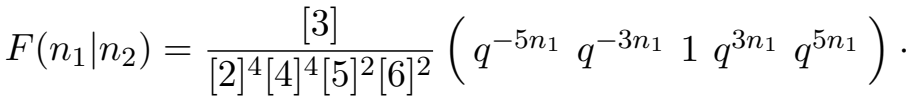

$$
\begin{aligned}
& \left(\begin{array}{ccccc}
0 & 1 & -\frac{[2][4][6]}{[3][5]} & \frac{[4]^{2}}{[2]^{2}} & -\frac{[3][6]}{[2][5]} \\
1 & 0 & -\frac{[2][4][6]}{[3]^{2}} & \frac{[5]}{[3]}\left(q^{4}+3+q^{-4}\right) & -\frac{[4]^{2}}{[2]^{2}} \\
\frac{[4][6]}{[5]} & -\frac{[2][4]}{[3]}\left(q^{4}+3+q^{-4}\right) & 2 \cdot \frac{[2]^{2}[4]^{2}[6]}{[3]^{2}[5]} & -\frac{[2][4]}{[3]}\left(q^{4}+3+q^{-4}\right) & \frac{[4][6]}{[5]} \\
-\frac{[4]^{2}}{[2]^{2}} & \frac{[5]}{[3]}\left(q^{4}+3+q^{-4}\right) & -\frac{[2][4][6]}{33]^{2}} & 0 & 1 \\
-\frac{[3][6]}{[2][5]} & \frac{[4]^{2}}{[2]^{2}} & -\frac{[2][4][6]}{[3][5]} & 1 & 0
\end{array}\right)\left(\begin{array}{c}
q^{-5 n_{2}} \\
q^{-3 n_{2}} \\
1 \\
q^{3 n_{2}} \\
q^{5 n_{2}}
\end{array}\right)
\end{aligned}
$$


The r.h.s. . of (9.2) is actually a Laurent polynomial in $q$. This formula is obtained by the evolution method of [70] with respect to the variables $n_{2}, n_{3}, n_{4}$, and it has the structure predicted by the general expression (8.1) for mutants. Also it vanishes whenever any of $n_{2}, n_{3}, n_{4}$ is unity. This is because the pretzel knots with unit length always commutes with any other length due to identities like (6.6). Eqs. (8.4)-(8.5) in section 8.3 are particular cases of (9.2).

\section{Conclusion}

In this paper we continued the study of the double fat tree realization of link diagrams, for which HOMFLY polynomials are described by an absolutely new and impressively effective formula (1.2). This formula requires redrawing knots as double fat tree diagram.

In the present paper, we showed that eq. (1.2), actually simplifies evaluation of knot polynomials of many knots presentable as two-bridge knots. It goes without saying that this overcomes most calculational complexities in knot/link theory. As an immediate illustration, we calculate a number of [21]-colored polynomials, and actually can now do this for many knots.

Particularly, this methodology helped us to explicitly illustrate/validate old expectations about the mutant pairs. Specifically, we explained the conditions when mutants are distinguishable or indistinguishable by [21]-colored polynomials. For instance, the KTC mutant pair can be resolved by these polynomials, but the pretzel mutants made from antiparallel fingers of odd lengths cannot be distinguished.

Of greatest importance for knot theory are now three questions:

- How large is the double fat tree family: what are the knots/links beyond it (if any?), and what is the way to find a double fat tree realization of a given knot/link?

- What is the origin of (1.2) and its effective field theory interpretation? What could be the meaning of quantization and loop diagrams in this effective theory.

- What is the $\beta$-deformation of (1.2), i.e. can this powerful formula be lifted to the super- and Khovanov-Rozansky polynomials?

Added to these could be two obvious technical next steps:

- to extend the double fat trees made from 4-strand braids propagators to arbitrary braids. The need for this for knot theory depends on the answer to the very first question. However, the problem itself can have its own value, especially because of its relation to multi-point conformal blocks. This approach will give complete solution provided we know the Racah matrices for arbitrary representations in the multi-point conformal blocks.

- to develop a systematic calculus for colored knots in arbitrary representations, beyond symmetric and [21]. This requires calculating the simplest Racah matrices describing the 2-bridge knots. We hope some of the non-rectangular representations may distinguish odd antiparallel pretzel mutants. 
Calculations in the case of representation [21] are quite involved and can be performed in slightly different ways. In particular, such technically independent exercise for the KTC mutants is reported in a parallel paper [90].

\section{Acknowledgments}

We are indebted to S.Nawata for participation at the early stage of the project and for critically reading the final version of manuscript. We also highly appreciate considerable help in calculations and discussions with S.Arthamonov and A.Sleptsov. We would like to thank the hospitality of the Indian Institute of Technology Bombay, where a part of this work was done.

Our work is partly supported by grants NSh-1500.2014.2, by RFBR grants 13-02-00457 (A.Mir.), 13-02-00478 (A.Mor.), 14-02-00627 (An.Mor.), by the joint grants 15-51-52031NSC-a (A.M.'s), 14-01-92691-Ind-a (A.M.'s), INT/RFBR/P-162 (P.R. and V.K.S.), by 1552-50041-YaF (A.M.'s) and by the Laboratory of Quantum Topology of Chelyabinsk State University through Russian Federation government grant 14.Z50.31.0020 (An.Mor.). Also we are partly supported by the Brazilian Ministry of Science, Technology and Innovation through the National Counsel of Scientific and Technological Development (A.Mor.).

\section{A Examples of [21]-colored HOMFLY}

Here we list examples of knots from the Rolfsen table of [48], for which the [21]-colored HOMFLY are now available.

\section{A.1 A list of knots with up to 10 intersections}

We start from the table which contains some relevant information about the knots up to 10 intersections.

The left part of the table lists the previously known cases:

- For the torus knots, the arbitrary colored HOMFLY polynomial is given by the Rosso-Jones formula [76].

- For three-strand knots, the [21]-colored HOMFLY polynomials can be calculated by the cabling method of [40] on ordinary computers. When the number of strands is three, we explicitly give a braid word, only instead of $\tau_{1}^{a_{1}} \tau_{2}^{a_{2}} \tau_{1}^{a_{3}} \ldots$ we write the sequence $a_{1}, a_{2}, a_{3}, \ldots$

- For two-bridge knots the knowledge of Racah matrices $S$ and $\bar{S}$ from [41] is sufficient: eq. (1.2) in this case reduces to an obvious matrix element $\mathcal{A}_{11}$. The corresponding answers are available from [41]. When the number of bridges is two, we explicitly give an $S-T$ word.

The right part of the table lists the cases which are available only now, by the method of the present paper, these knots are boldfaced in the first column. Numerous intersections between the left and right parts of the table are important for checking our conjecture (1.2). 
An exhaustive list of the pretzel knots up to 10 intersections is borrowed from the third paper of ref. [45]. They are distinguished from generic double fat tree knots only by simplicity of computer calculations, what is actually quite important.

The starfish cases are also usually simple enough: they still involve just one sum over representations (all pretzel knots are automatically starfish). The cases with two and three propagators involve two and three such sums and are considerably more difficult for computers. A search for maximally simple representations are therefore important from this point of view, and hopefully the table can be significantly improved.

The right part of the table is currently incomplete, but it seems all the knots with 10 or less intersections to fit into it (with the possible exception of $10_{161}$, which is anyhow present in the left part of the table).

In addition to this table, answers are available for some explicitly identified mutants with 11 intersections. 


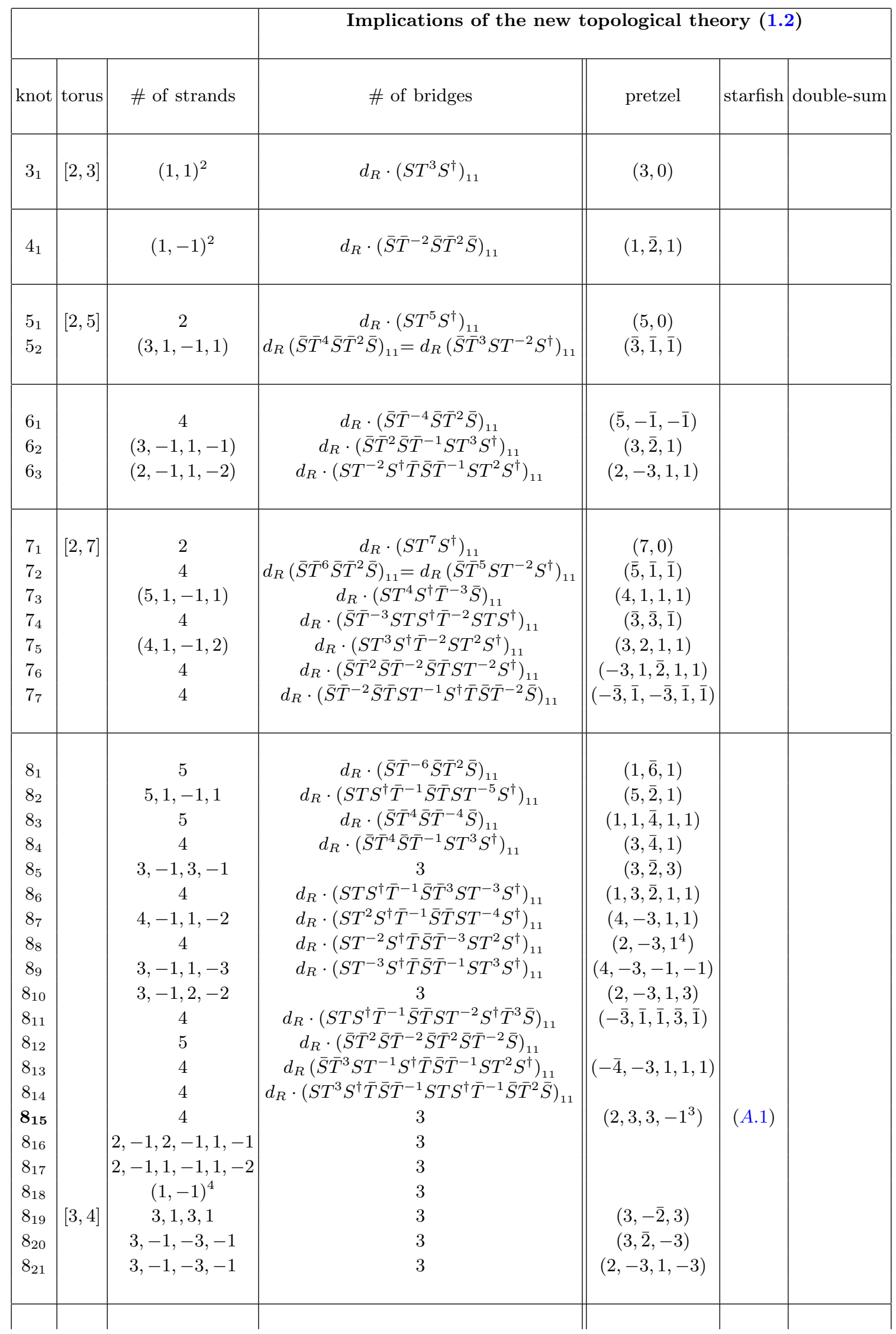




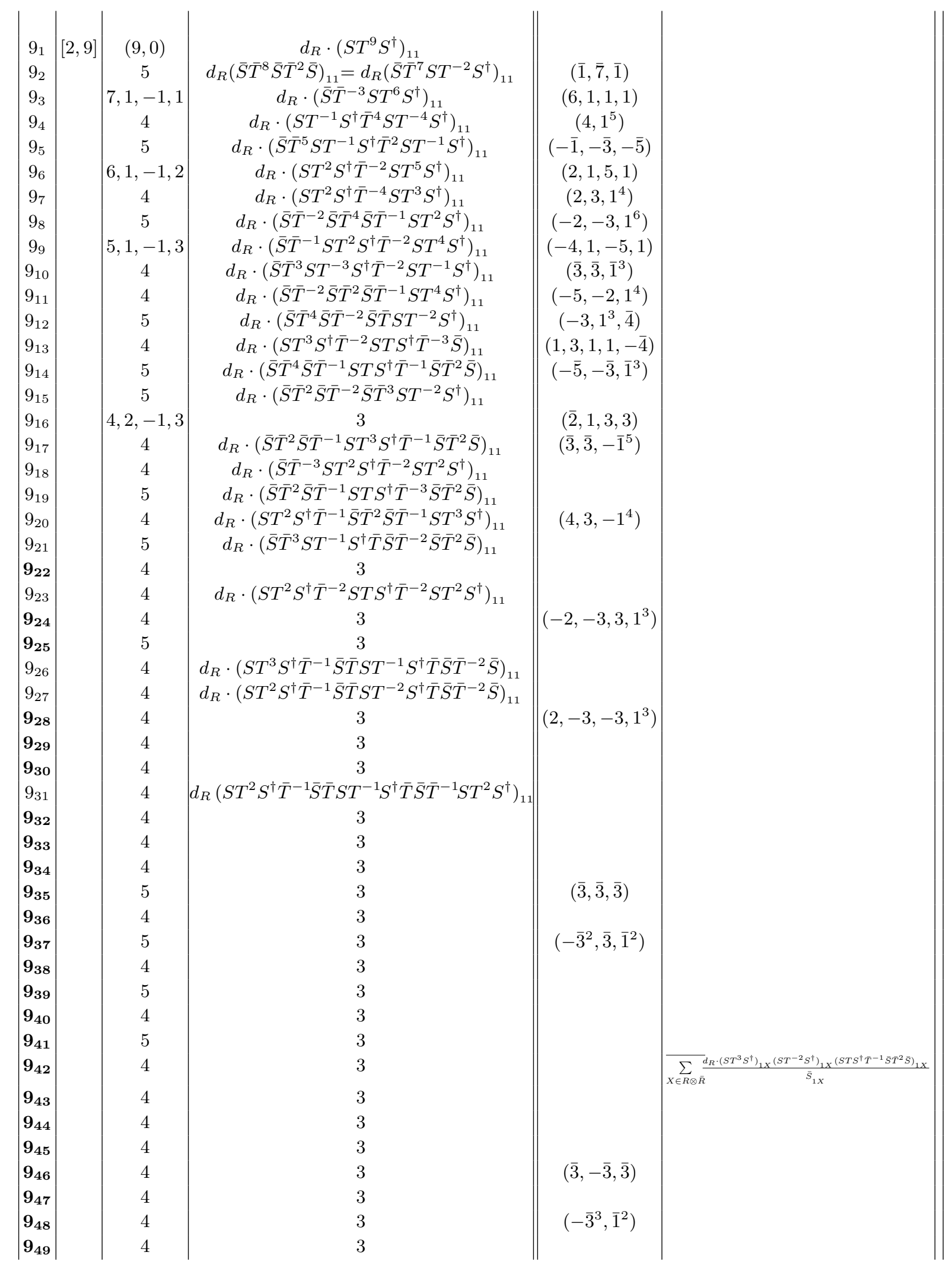




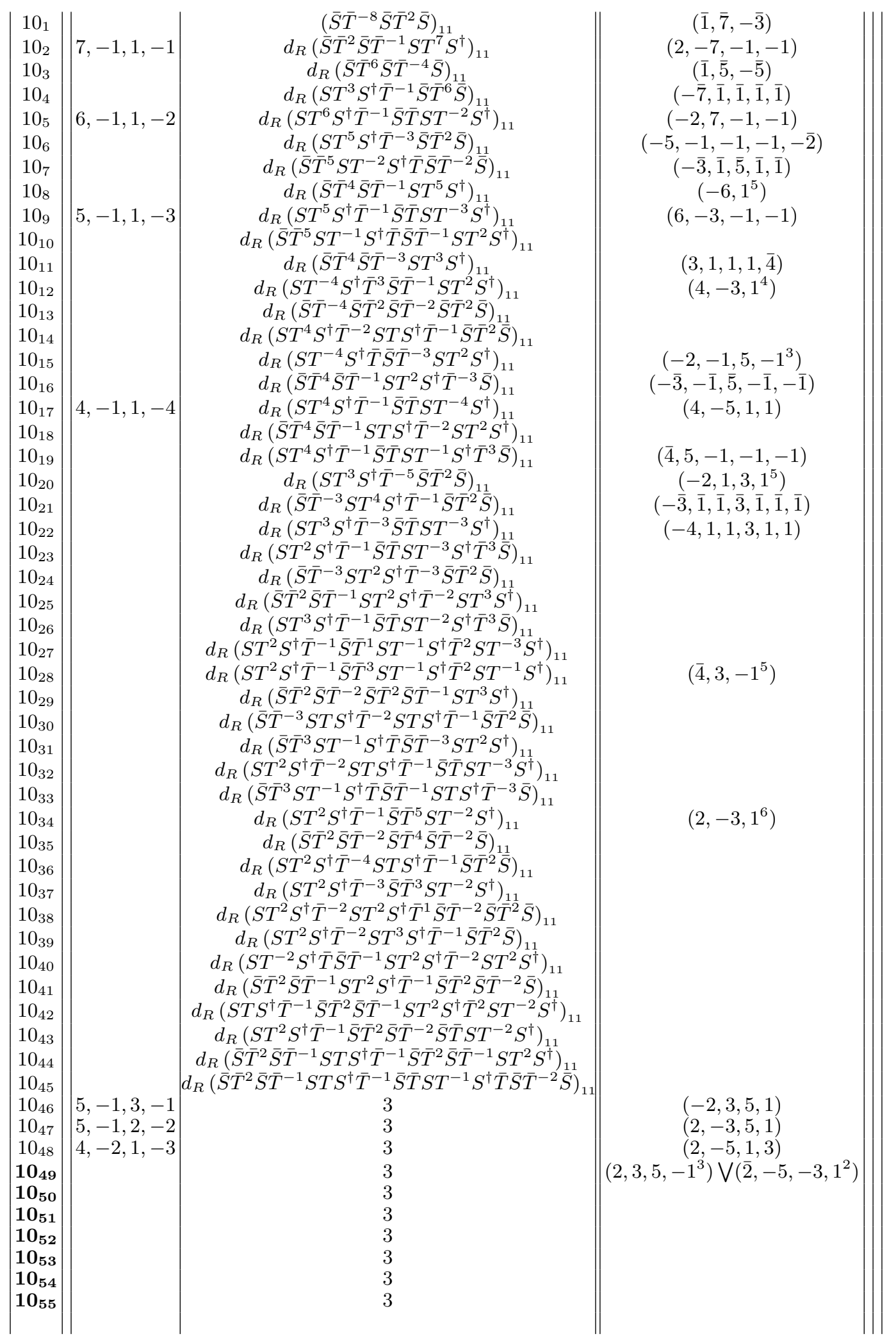




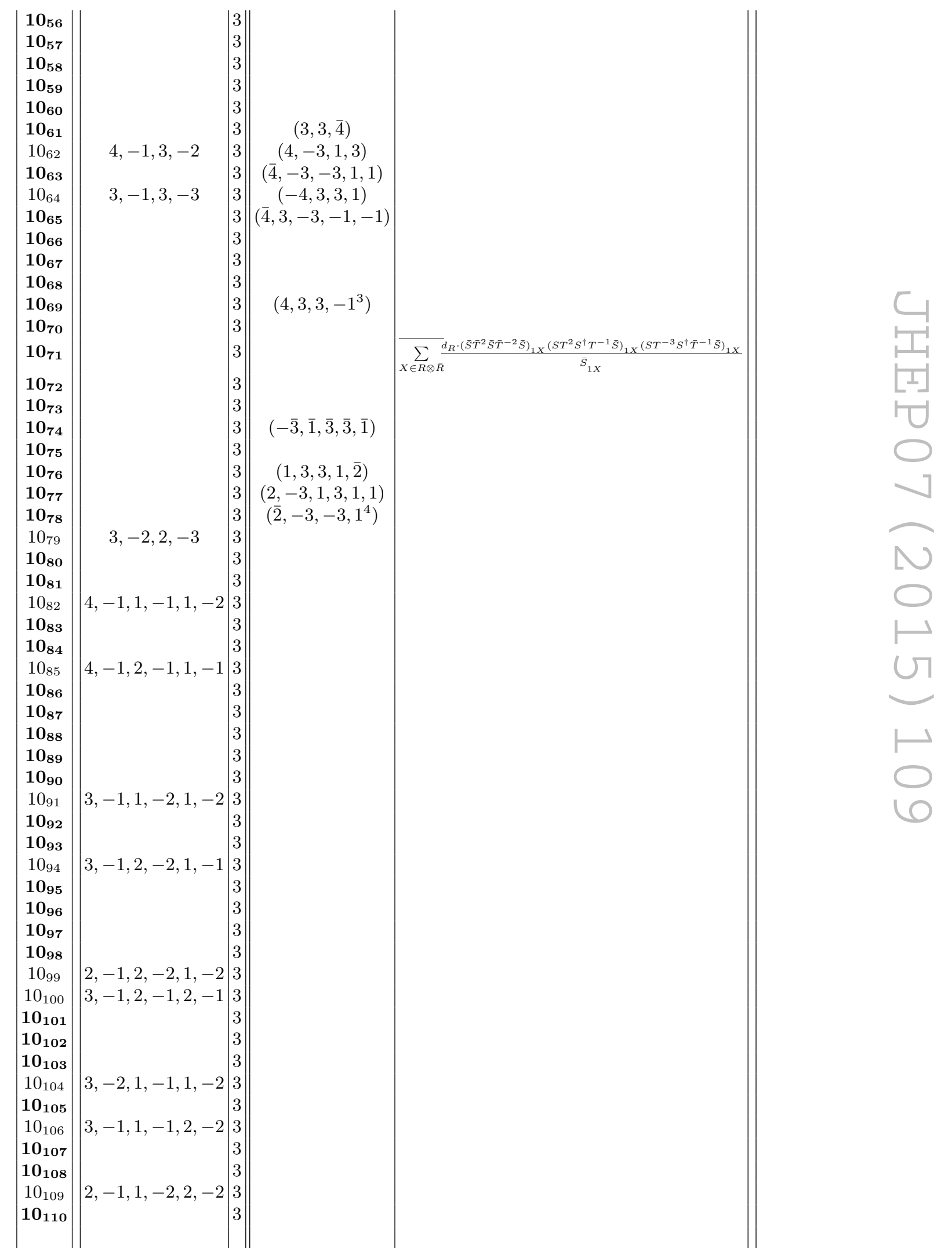




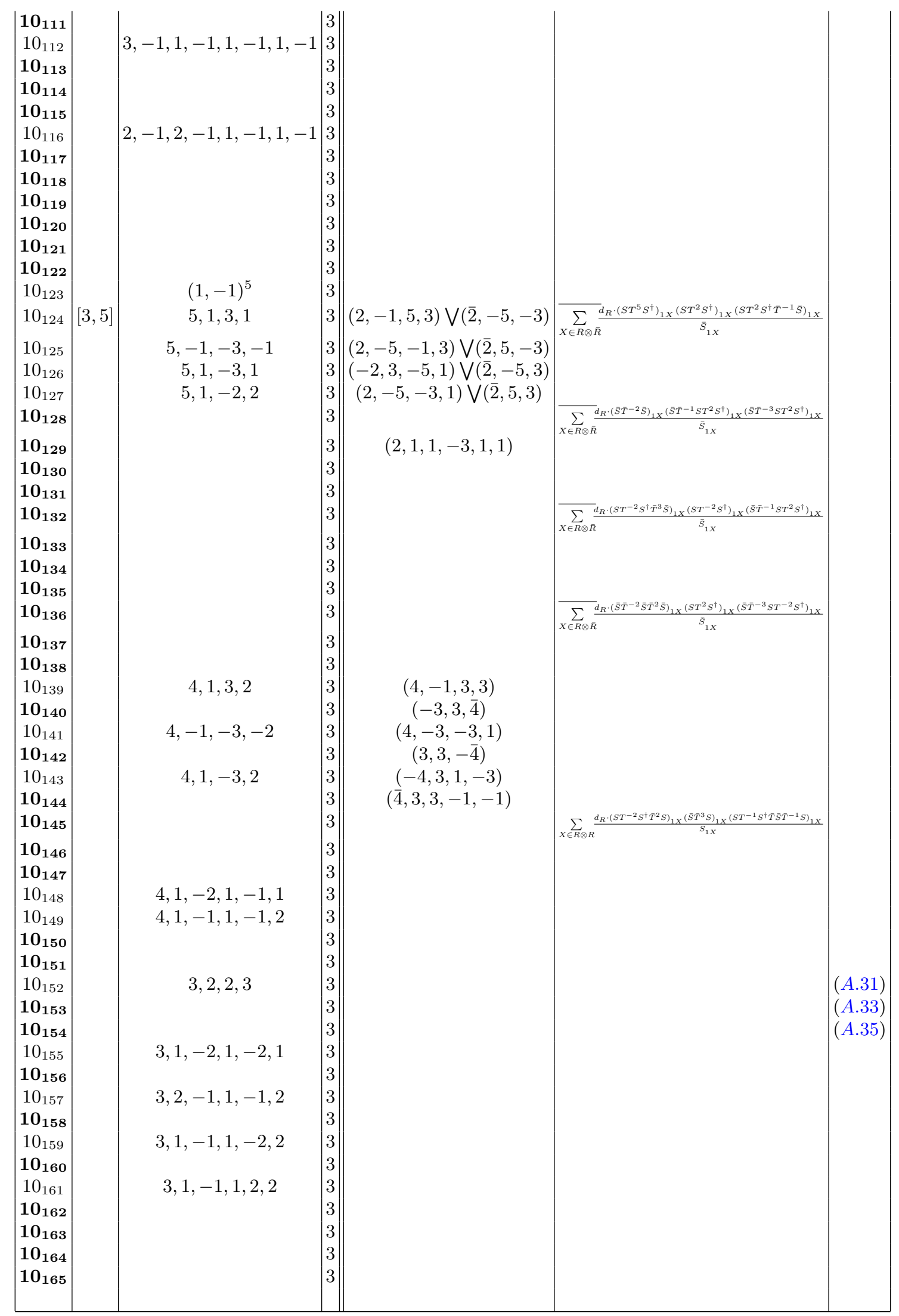




\section{A.2 Some thin knots}

A lot of explicit examples with less than nine intersections can be found in [40] and [41]. Here we add the only 8 -intersection knot 815 which was not present in those lists (it is pretzel, but possesses also a simpler triple-finger realization),

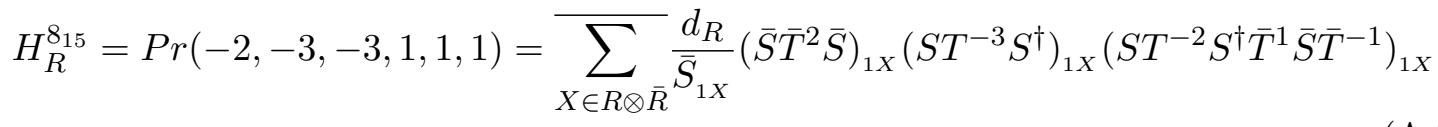

and a couple of 10-intersection knots:

$$
H_{R}^{10_{71}}=\overline{\sum_{X \in R \otimes \bar{R}}} \frac{d_{R}}{\bar{S}_{1 X}}\left(\bar{S} \bar{T}^{2} \bar{S} \bar{T}^{-2} \bar{S}\right)_{1 X}\left(S T^{2} S^{\dagger} \bar{T}^{-1} \bar{S}\right)_{1 X}\left(S T^{-3} S^{\dagger} \bar{T}^{-1} \bar{S}\right)_{1 X}
$$

and

$$
\begin{gathered}
H_{R}^{10_{125}=} \bar{\sum}_{X \in R \otimes \bar{R}} \frac{d_{R}}{\bar{S}_{1 X}^{2}}\left(S T^{-5} S^{\dagger}\right)_{1 X}\left(S T^{-1} S^{\dagger}\right)_{1 X}\left(S T^{3} S^{\dagger}\right)_{1 X}\left(S T^{2} S^{\dagger}\right)_{1 X} \\
H_{[21]}^{8_{15}}=\frac{1}{q^{24} A^{30}} .
\end{gathered}
$$

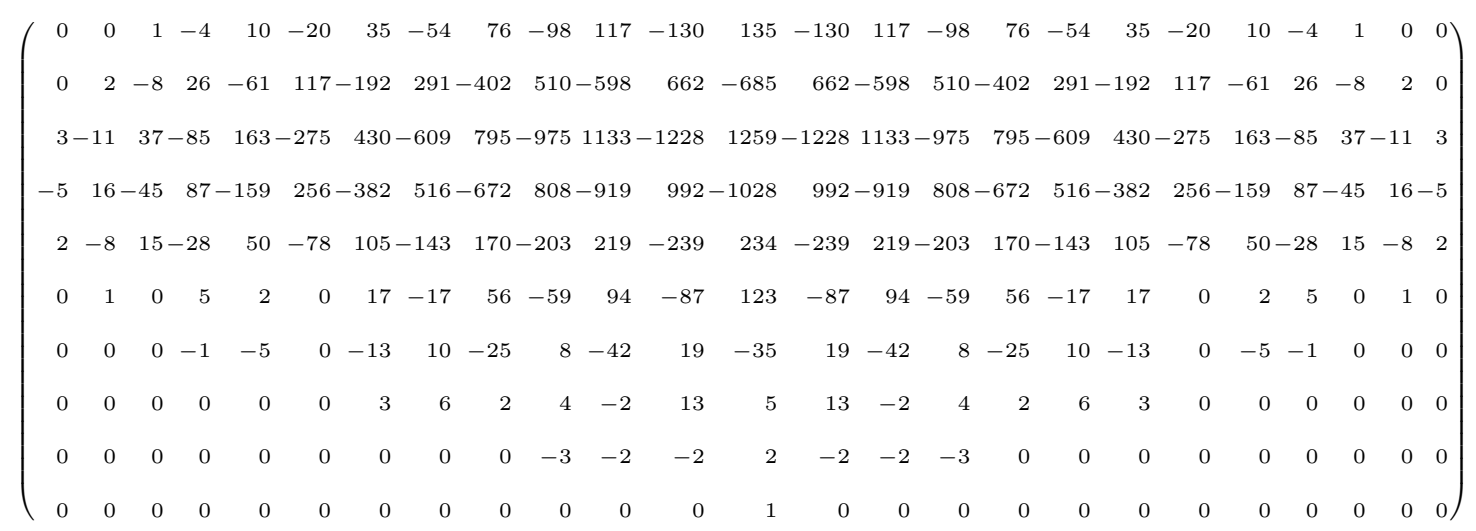




$$
\begin{aligned}
& \text { 垔 }
\end{aligned}
$$

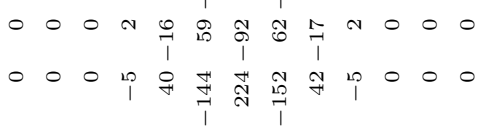

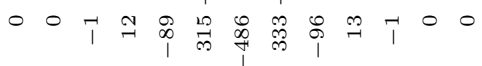

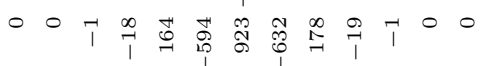

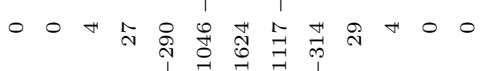

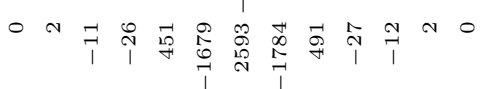

$$
\begin{aligned}
& \text { - }
\end{aligned}
$$

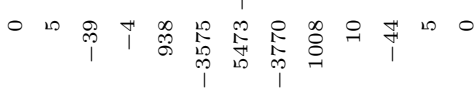

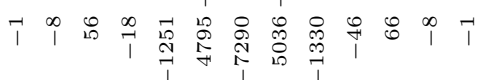

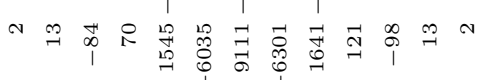

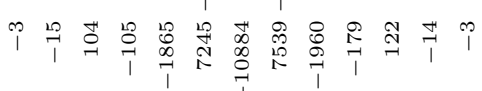

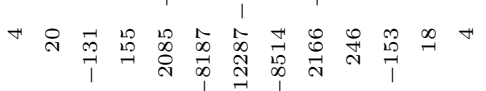

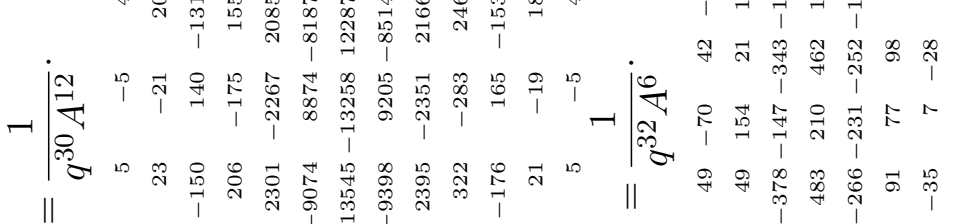

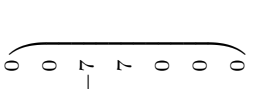
- or oroo

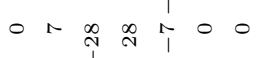

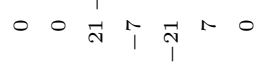

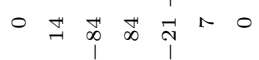

个。

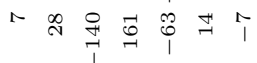

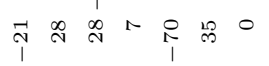

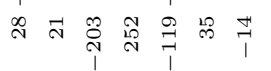

卉乐

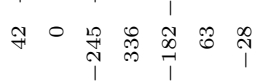

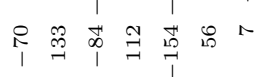

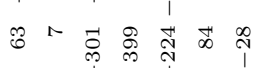

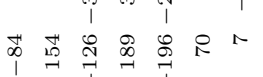

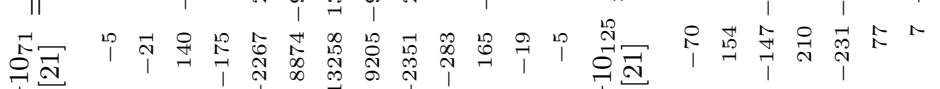

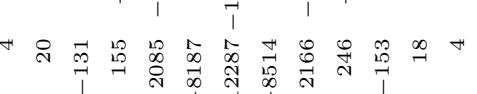

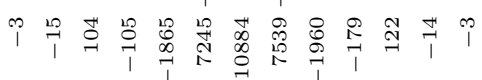

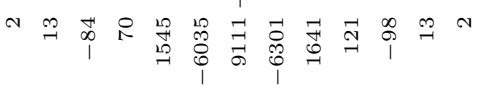

$\vec{\imath} \begin{aligned} & \infty \\ & 1\end{aligned}$

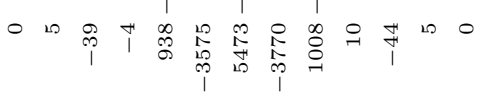

○

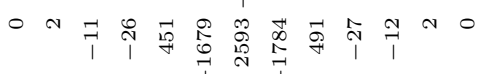

○。

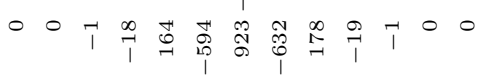

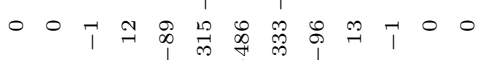

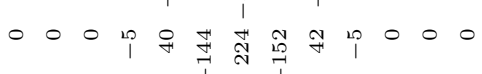

○。ㅅํำ ป

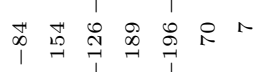

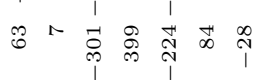

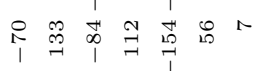

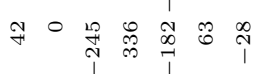

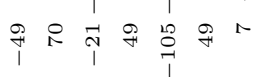

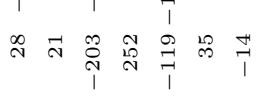

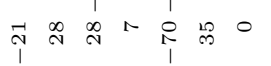

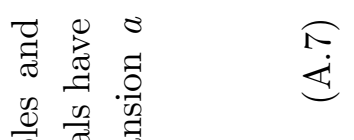

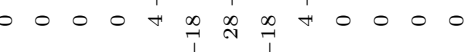

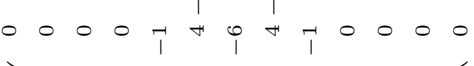
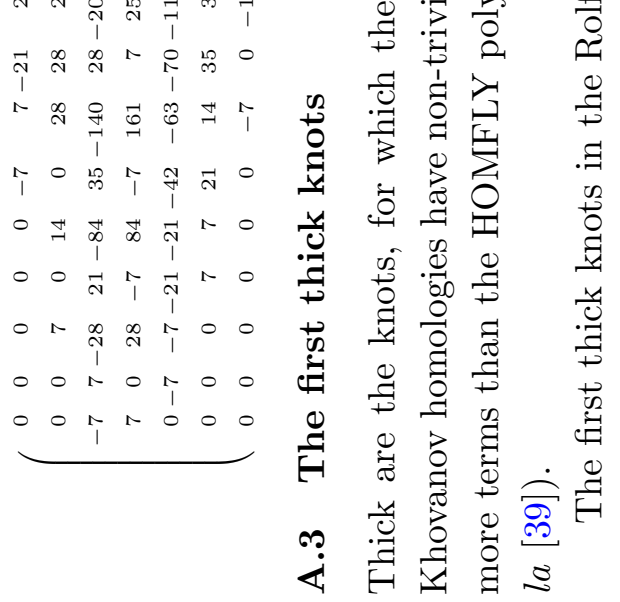

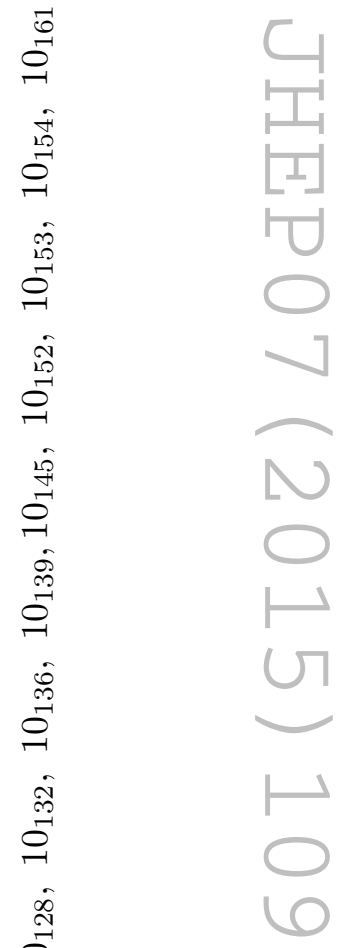




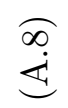

$\stackrel{\widehat{a}}{\dot{s}}$

700000

- H H 00000

त

H H

700000

$0 \overrightarrow{1}+0000$

त

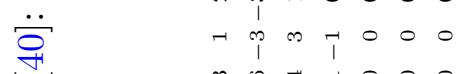

$\infty \begin{array}{lllll}1 & 1 & 0 & 0 & 0\end{array}$

$\rightarrow i \infty \infty 00$

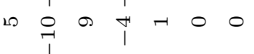

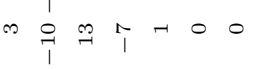

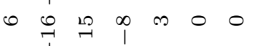

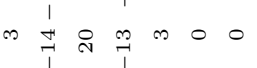

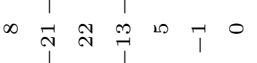

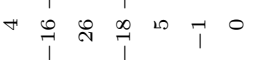

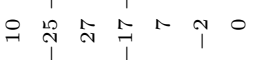

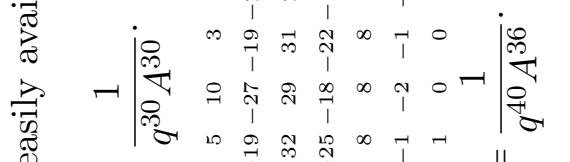

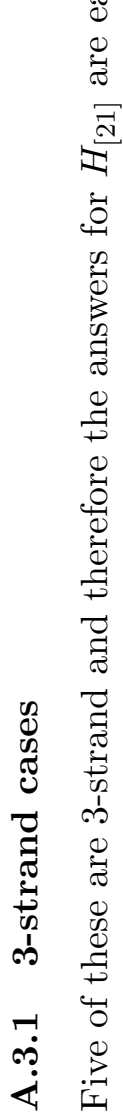

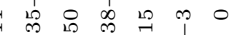

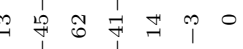

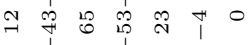

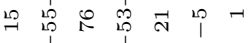

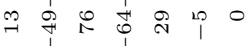

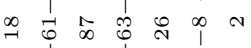

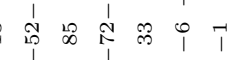

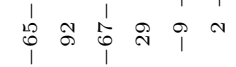

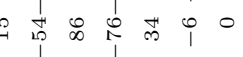

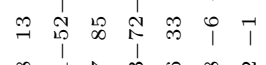

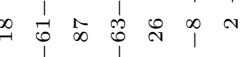

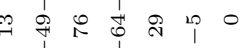

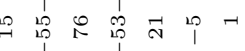

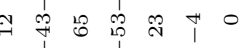

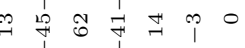
$\exists$ 品品品只只。

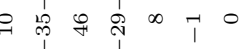

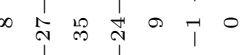

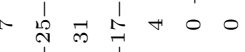
ก b 일 $\Rightarrow \forall p+00$

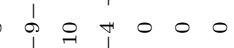

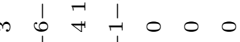

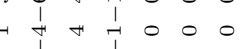

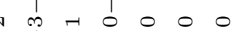
$\begin{aligned} & 1 \\ & -1\end{aligned}-0000$

$\begin{array}{lllll}1 & \infty & \infty & 0 & 0\end{array}$

$r \infty n \begin{array}{llll}n & 0 & 0 & 0\end{array}$

N $\boldsymbol{p} \rightarrow 000$

- 1 r 00000

700000 $\widehat{\exists}$

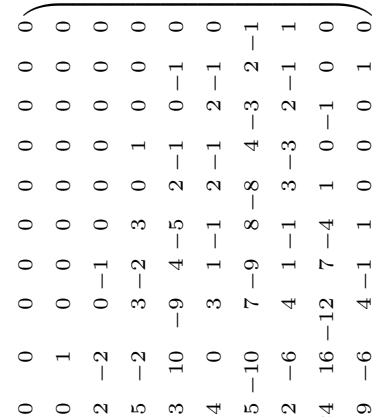
$\infty$ 莒

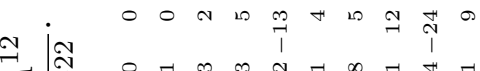

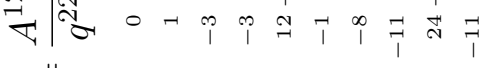
$\| \overrightarrow{1}$ | 1 \%

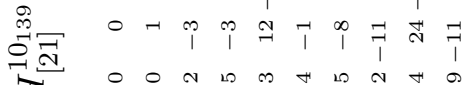

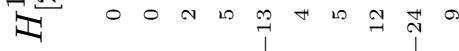

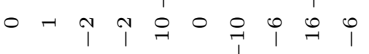
O O O m D

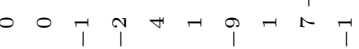

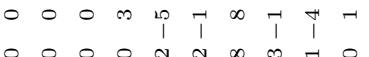

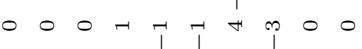
$\begin{array}{lllllllllllll}0 & 0 & 0 & 0 & 0 & h\end{array}$

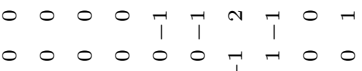




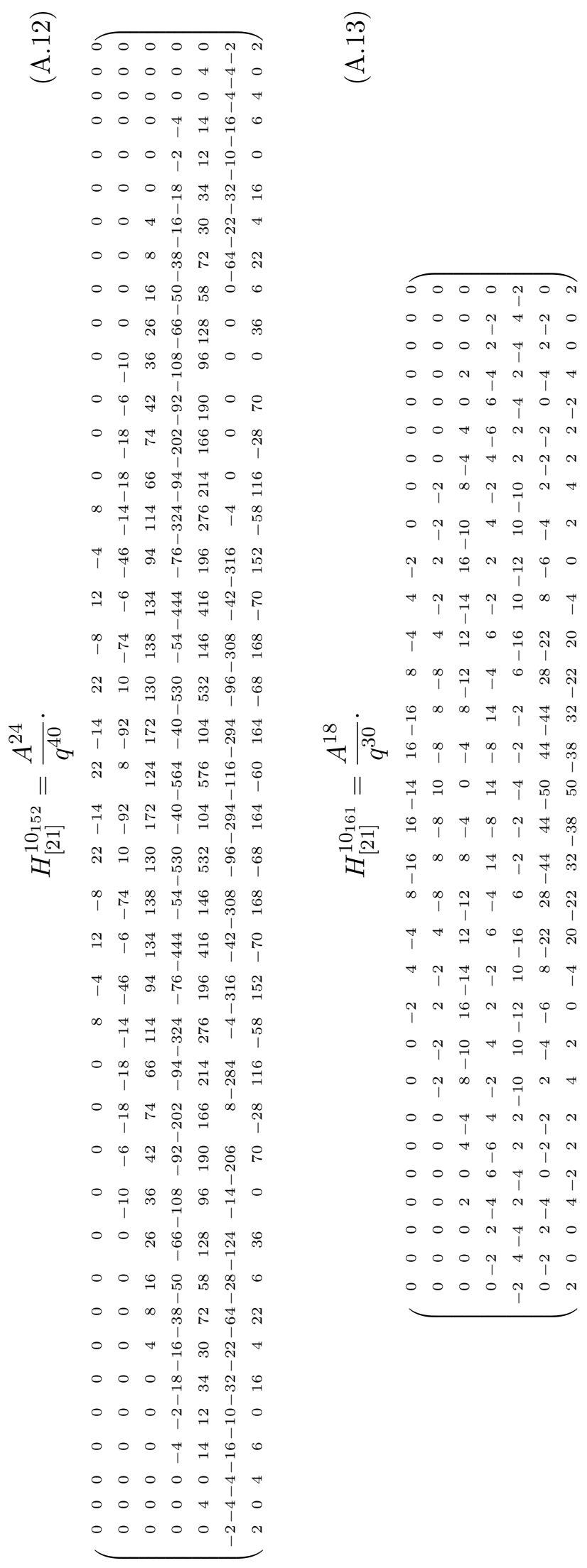


Moreover, two of these are torus knots, $8_{19}=$ Torus $[3,4]$ and $10_{124}=$ Torus $[3,5]$, and therefore arbitrary colored HOMFLY polynomials for them are available: provided by the Rosso-Jones formula [13, 76].

\section{A.3.2 4-parallel pretzel finger cases}

Three of the above thick knots are of the pretzel type and are described by the simple formulas:

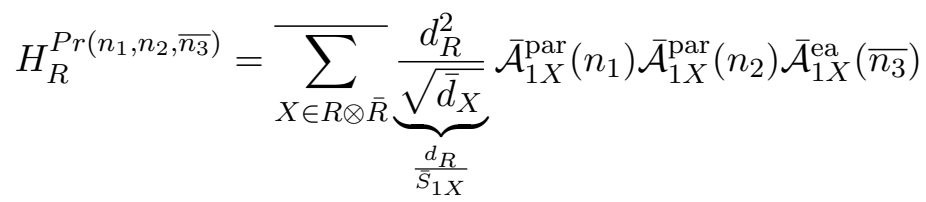

and

$$
H_{R}^{P r\left(n_{1}, n_{2}, n_{3}, n_{4}\right)}=\bar{\sum}_{X \in R \otimes \bar{R}} \underbrace{\frac{d_{R}^{3}}{\bar{d}_{X}}}_{\frac{d_{R}}{\bar{S}_{1 X}^{2}}} \overline{\mathcal{A}}_{1 X}^{\mathrm{par}}\left(n_{1}\right) \overline{\mathcal{A}}_{1 X}^{\mathrm{par}}\left(n_{2}\right) \overline{\mathcal{A}}_{1 X}^{\mathrm{par}}\left(n_{3}\right) \overline{\mathcal{A}}_{1 X}^{\mathrm{par}}\left(n_{4}\right)
$$

These three cases are 3 -strand (and thus already known)

$$
\begin{aligned}
& 8_{19}=\text { Torus }[3,4]=\operatorname{Pr}(3,3,-\overline{2}) \\
& 10_{124}=\text { Torus }[3,5]=\operatorname{Pr}(5,3,-\overline{2})=\operatorname{Pr}(2,-1,5,3) \quad \Longrightarrow \quad H^{8_{19}}=(A .8) \\
& H^{10_{124}}=(A .10)
\end{aligned}
$$

and

$$
10_{139}=\operatorname{Pr}(4,-1,3,3) \quad \Longrightarrow \quad H^{10_{139}}=(A .3 .1)
$$

\section{A.3.3 Realizations from [43]}

According to [42] and [43], seven thick knots from [11] can be realized just as triple-finger starfish diagrams:

$$
\begin{aligned}
& H_{R}^{8_{19}}=\overline{\sum_{X \in R \otimes \bar{R}}} \frac{d}{R}_{\bar{S}_{1 X}}\left(S T^{3} S^{\dagger}\right)_{1 X}\left(S T^{3} S^{\dagger}\right)_{1 X}\left(\bar{S} \bar{T}^{-2} \bar{S}\right)_{1 X} \quad \Longrightarrow \quad H_{R}^{8_{19}}=(A .8) \\
& H_{R}^{9_{42}}=\overline{\sum_{X \in R \otimes \bar{R}}} \frac{d_{R}}{\bar{S}_{1 X}}\left(S T^{3} S^{\dagger}\right)_{1 X}\left(S T^{-2} S^{\dagger}\right)_{1 X}\left(S T S^{\dagger} \bar{T}^{-1} \bar{S} \bar{T}^{2} \bar{S}\right)_{1 X} \\
& H_{[21]}^{942}=\frac{1}{q^{22} A^{8}} . \\
& \left(\begin{array}{rrrrrrrrrrrrrrrrrrrrrrr}
0 & 0 & 0 & 0 & 0 & 0 & 1 & 0 & 2 & -1 & 2 & 0 & 2 & -1 & 2 & 0 & 1 & 0 & 0 & 0 & 0 & 0 & 0 \\
0 & 0 & 0 & -1 & -1 & -2 & -1 & -2 & -3 & -3 & -4 & -2 & -4 & -3 & -3 & -2 & -1 & -2 & -1 & -1 & 0 & 0 & 0 \\
0 & 1 & 1 & 1 & 3 & 2 & 6 & 2 & 8 & 4 & 9 & 4 & 9 & 4 & 8 & 2 & 6 & 2 & 3 & 1 & 1 & 1 & 0 \\
-1 & 1 & -5 & 3 & -9 & 4 & -15 & 5 & -19 & 5 & -21 & 5 & -21 & 5 & -19 & 5 & -15 & 4 & -9 & 3 & -5 & 1 & -1 \\
1 & -2 & 4 & -3 & 9 & -6 & 14 & -7 & 20 & -10 & 24 & -10 & 24 & -10 & 20 & -7 & 14 & -6 & 9 & -3 & 4 & -2 & 1 \\
0 & 0 & 0 & 0 & -1 & 0 & -4 & 1 & -8 & 4 & -12 & 4 & -12 & 4 & -8 & 1 & -4 & 0 & -1 & 0 & 0 & 0 & 0 \\
0 & 0 & 0 & 0 & -1 & 1 & -1 & 0 & 3 & -1 & 5 & -4 & 5 & -1 & 3 & 0 & -1 & 1 & -1 & 0 & 0 & 0 & 0 \\
0 & 0 & 0 & 0 & 0 & 0 & 0 & 1 & -1 & 2 & -3 & 2 & -3 & 2 & -1 & 1 & 0 & 0 & 0 & 0 & 0 & 0 & 0
\end{array}\right)
\end{aligned}
$$


雪要

重 亭
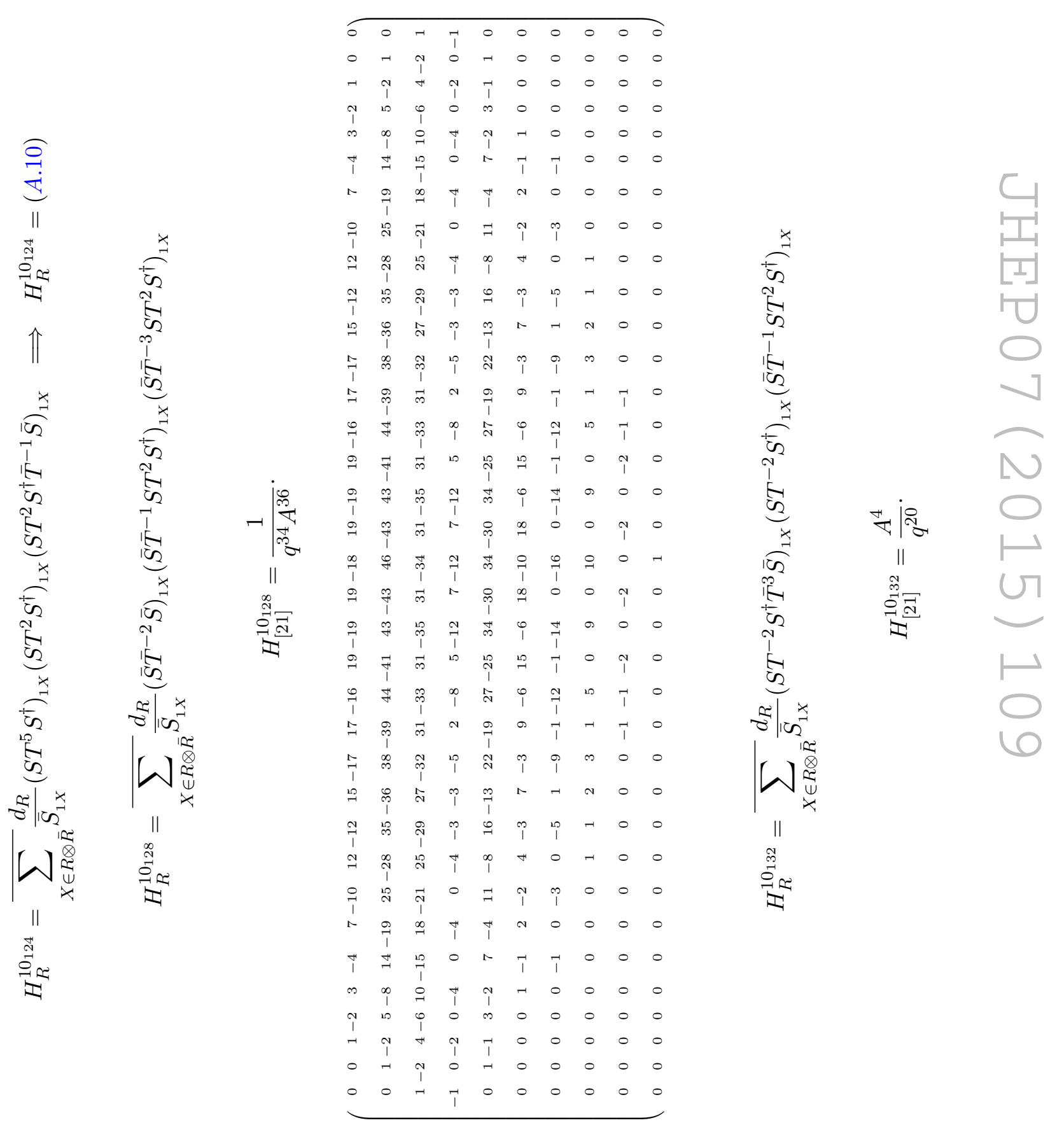


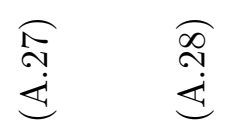

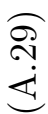

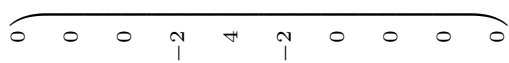

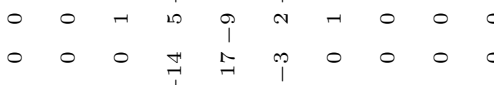

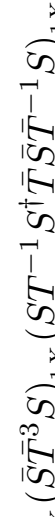

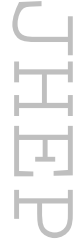

$\begin{array}{lllllllllllllllll}0 & 0 & 0 & 0 & -1 & 7 & 0 & 0\end{array}$

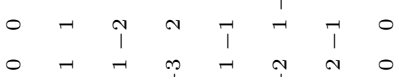

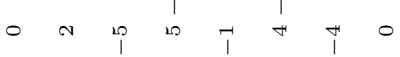

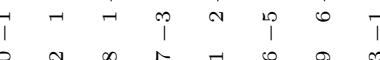

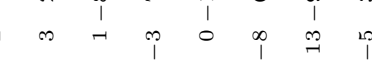

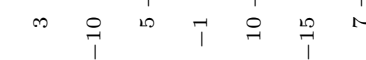

$+\neg 0 \quad \exists I i$

- N

1

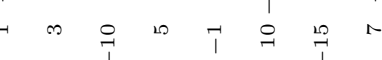

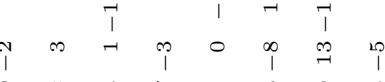

$\begin{array}{lllllll}1 & \infty & 1 & -1 & 0 & 0 & 1 \\ & 1 & 0 & 1 & 0 & 1 & 0\end{array}$

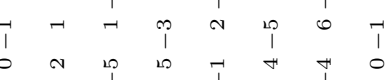

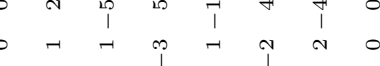

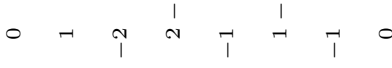

$\begin{array}{cccccccc}0 & 0 & 0 & 0 & -1 & -1 & 0 & 0 \\ 0 & 0 & 1 & 0 & 0 & -1 & 0 & 0\end{array}$

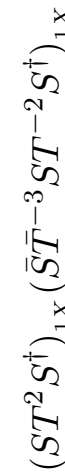

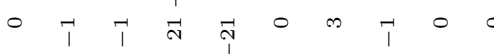

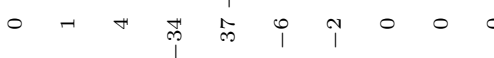

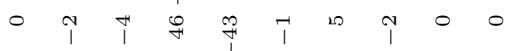

$\neg \infty \exists \hat{b}$ 品

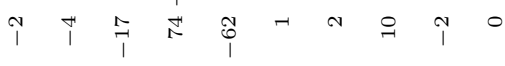

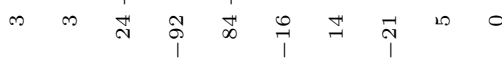

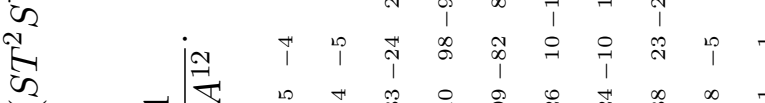

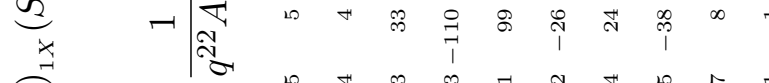

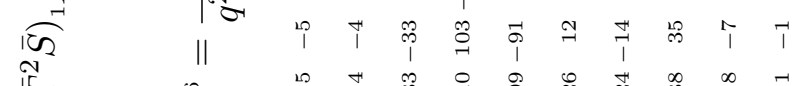

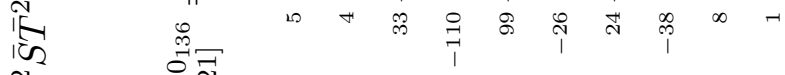

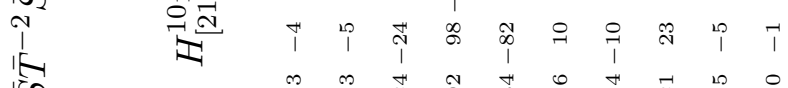

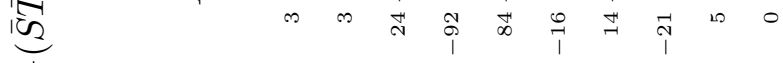

$\sqrt{2}$

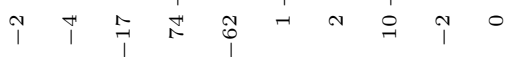

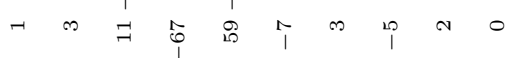

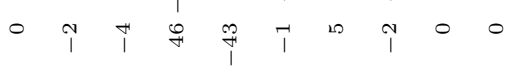

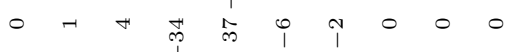

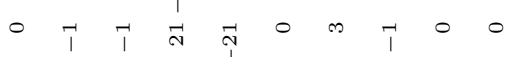

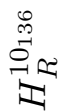

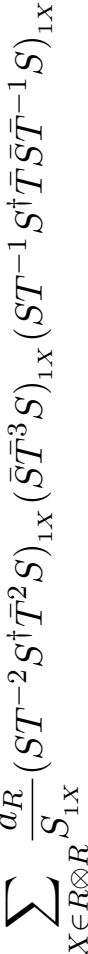

000 藏

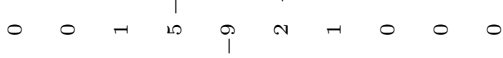

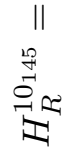




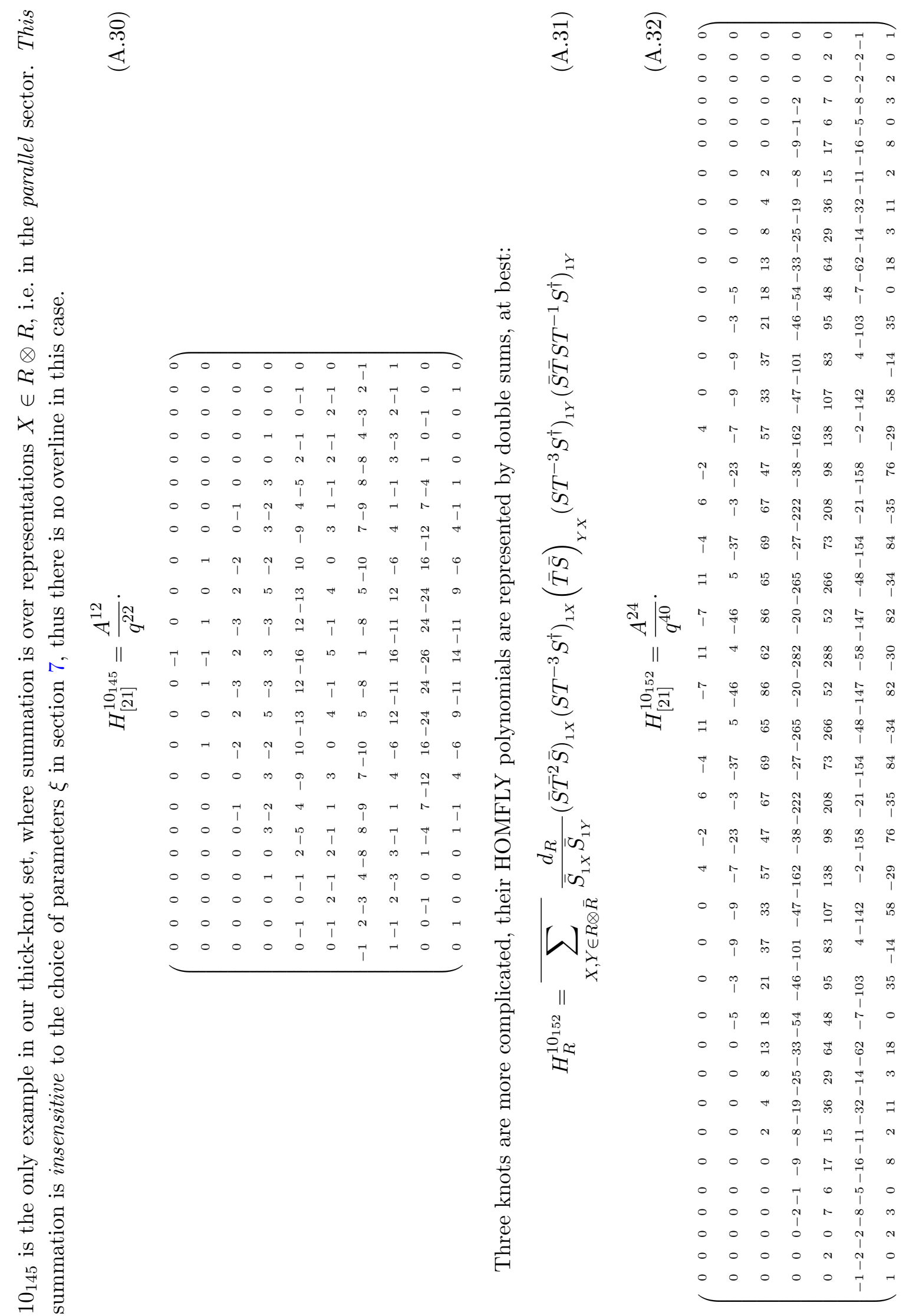




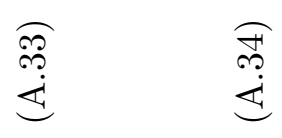

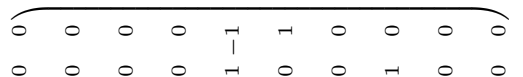

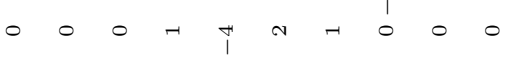

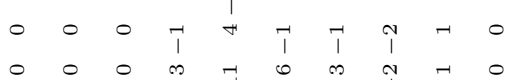

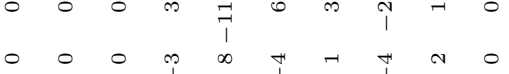

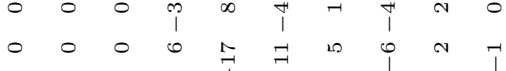

- 0 - 0 D

- 0 小 0 ช

कि

- o

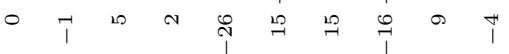

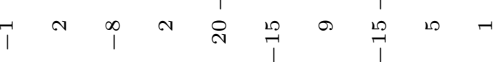

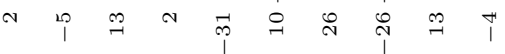

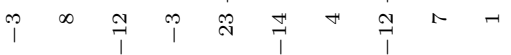

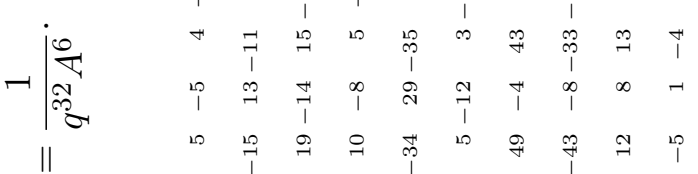

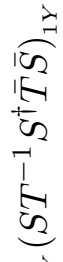

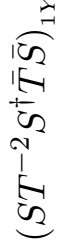

L

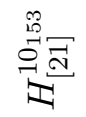

* F

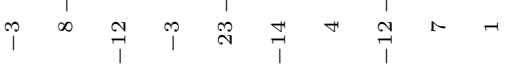

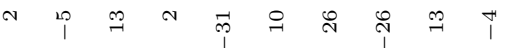

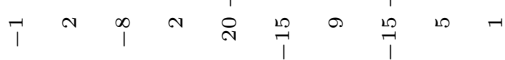

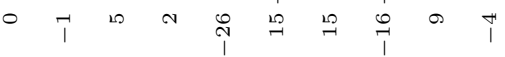

- 0 o

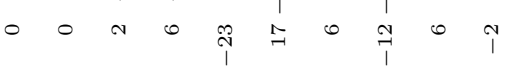

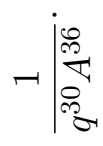

(5)

离

कू

$\left.\left.\right|^{2}\right|_{102} ^{2}$

- 0 - 0 D

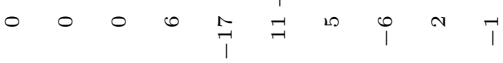

$\sqrt{x}$

记

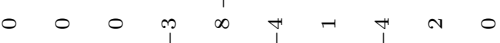

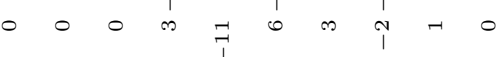

॥

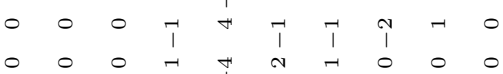

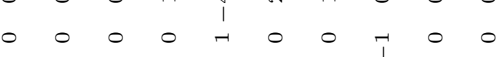

$00001+1000$

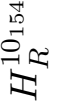



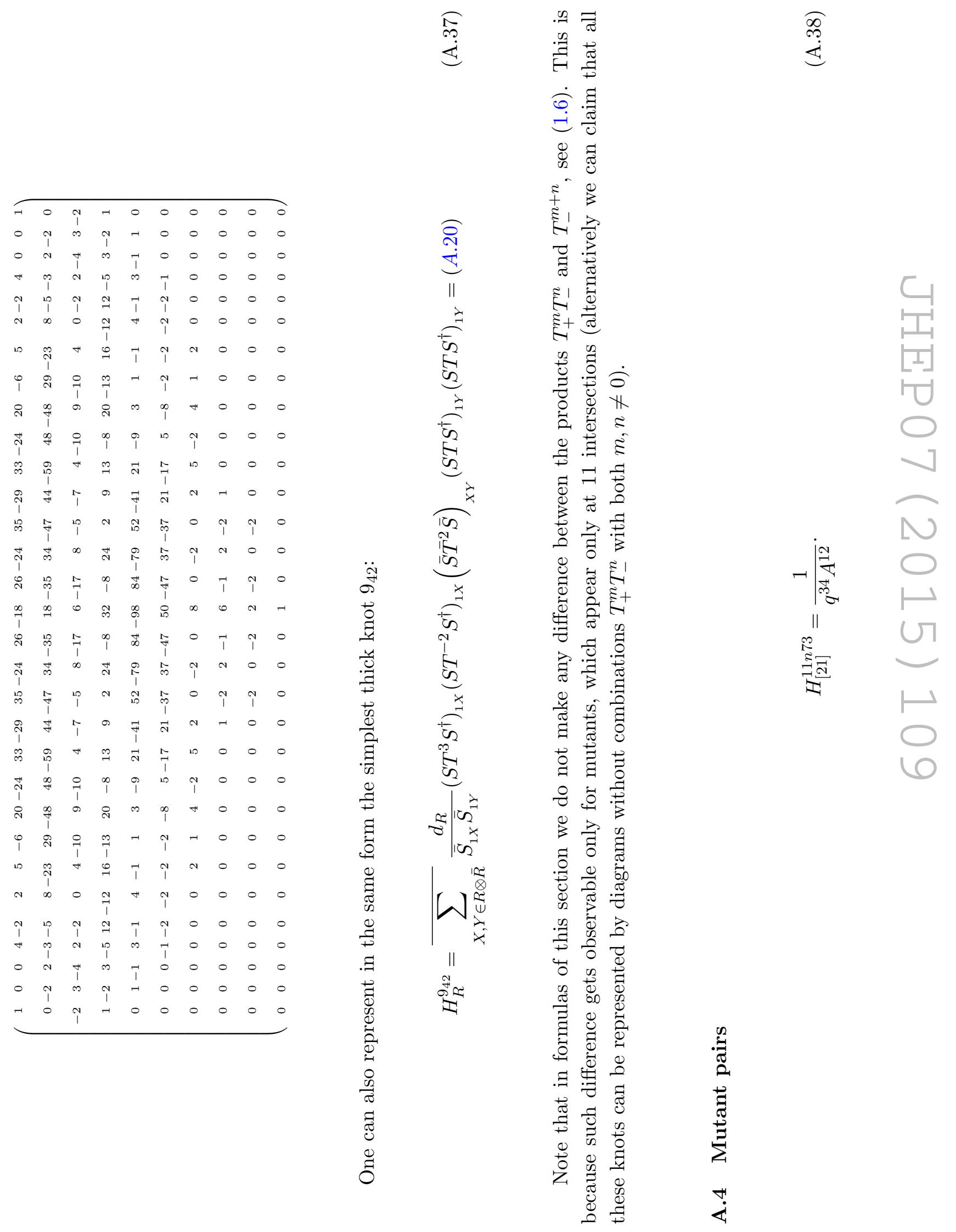


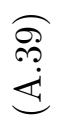

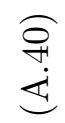

\begin{tabular}{|c|c|c|c|c|c|c|c|c|}
\hline & ${ }^{\circ}$ & & $n$ & $i$ & & $\vec{\imath}$ & 0 & \\
\hline & $:$ & $\stackrel{\circ}{\sim}$ & $\underset{R}{\stackrel{1}{r}}$ & $\begin{array}{l}1 \\
0 \\
0\end{array}$ & $\stackrel{0}{\wedge}$ & $\begin{array}{l}1 \\
0 \\
10\end{array}$ & 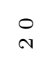 & 0 \\
\hline & 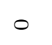 & $\vec{t}$ & $\infty$ & i & $\infty$ & $\infty$ & $\rightarrow$ & 0 \\
\hline & $r$ & $\begin{array}{l}\infty \\
1 \\
1\end{array}$ & $\stackrel{\sim}{ }$ & ते & 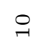 & $\begin{array}{l}\infty \\
1\end{array}$ & i & \\
\hline & ๙ & $i$ & $\infty$ & $\stackrel{1}{a}$ & ळి & $\approx$ & 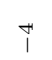 & \\
\hline & $\forall$ & ㄱ. & 모 & $\infty$ & $\neg$ & $\mathbb{J}$ & $\stackrel{\infty}{\sim}$ & i \\
\hline & $r$ & 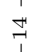 & $\bumpeq$ & $\begin{array}{c}\infty \\
\infty \\
\infty\end{array}$ & $\stackrel{\infty}{\infty}$ & $\infty$ & $i$ & \\
\hline & 10 & J & $\stackrel{\mathscr{D}}{\Rightarrow}$ & م̂. & $\stackrel{\circ}{\rightarrow}$ & $\vec{I}$ & $\check{\infty}$ & 10 \\
\hline & iे & $\vec{p}$ & $\vec{\lambda}$ & $\overrightarrow{\vec{g}}$ & $\stackrel{\infty}{\rightarrow}$ & $\overrightarrow{\vec{p}}$ & $\stackrel{0}{i}$ & $i_{i}$ \\
\hline & ๙ & $\underset{\infty}{\infty}$ & 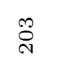 & 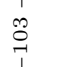 & $\stackrel{1}{1}$ & 品 & 多 & \\
\hline & $\mathscr{F}$ & $\underset{i}{\infty}$ & $\exists$ & $\vec{N}$ & :ु & $\overrightarrow{\dot{i}}$ & $\overrightarrow{7}$ & $\cong$ \\
\hline & $\infty$ & Iิ & $\underset{N}{\stackrel{R}{N}}$ & 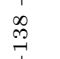 & $\stackrel{\infty}{\rightarrow}$ & $\stackrel{ }{\stackrel{-}{2}}$ & ช & ๙ \\
\hline & 용 & $\overrightarrow{\mid \vec{\infty}}$ & $\vec{\sigma}$ & స్లి & స్లె & $\stackrel{\infty}{i}$ & $\stackrel{\sim}{\mp}$ & 品 \\
\hline & $\stackrel{9}{1}$ & $\stackrel{8}{g}$ & :్లి & : & กี & $\underset{I}{D}$ & $œ$ & ig \\
\hline & 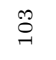 & $\stackrel{8}{2}$ & $\vec{\Xi}$ & 足 & 品 & $\underset{i}{\stackrel{H}{*}}$ & 点 & $\vec{\sigma}$ \\
\hline & $\stackrel{\infty}{i}$ & $\underset{\sim}{\infty}$ & హ్లి & 속 & $\stackrel{\infty}{\infty}$ & స్ & $\stackrel{\mathfrak{o}}{\sigma}$ & . \\
\hline & $\stackrel{\rightarrow}{\rightarrow}_{-1}^{\infty}$ & 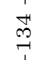 & $\stackrel{\substack{\infty \\
\sim}}{\sim}$ & $\stackrel{\infty}{\mathcal{J}}$ & $\underset{\infty}{\mathbb{\infty}}$ & $\approx$ & $\tilde{p}_{1}^{0}$ & $\stackrel{\square}{\circ}$ \\
\hline & $\underset{\sim}{\infty}$ & $\begin{array}{l}1 \\
\underset{J}{\infty}\end{array}$ & హ్లి & ي & $\tilde{\infty}$ & స్ & $\stackrel{\mathfrak{O}}{\circ}$ & e \\
\hline & 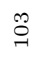 & $\stackrel{\circ}{\stackrel{9}{1}}$ & $\overrightarrow{\breve{f}}$ & 足 & 蛧 & $\stackrel{\vec{T}}{1}$ & 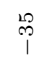 & $\vec{\sigma}$ \\
\hline & $\stackrel{\rho}{\overrightarrow{1}}$ & 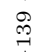 & $\stackrel{\text { లె }}{ }$ & : & 活 & I & 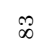 & 品 \\
\hline & $\stackrel{10}{R}$ & $\vec{\infty}$ & $\vec{\sigma}$ & ڤે & స్లి & 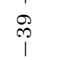 & $\stackrel{\sim}{7}$ & 吕 \\
\hline & $\begin{array}{l}\infty \\
1\end{array}$ & 워 & $\underset{\sim}{\stackrel{D}{N}}$ & 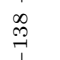 & $\stackrel{\infty}{\rightarrow}$ & $\stackrel{\circ}{\stackrel{1}{1}}$ & $\tilde{\sigma}$ & $\stackrel{8}{\circ}$ \\
\hline & $\mathscr{F}$ & $\stackrel{\infty}{\underset{J}{1}}$ & $\exists$ & $\overrightarrow{\mathrm{N}}$ & :ु్ & $\vec{i}$ & $\overrightarrow{7}$ & $\hat{i}$ \\
\hline & $\sim$ & $\infty_{1}^{\infty}$ & 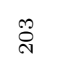 & 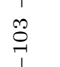 & i & 品 & 骂 & 0 \\
\hline & จे & $\vec{p}$ & $\vec{\sim}$ & $\vec{g}$ & $\stackrel{\infty}{\rightarrow \infty}$ & $\overrightarrow{\vec{p}}$ & $\stackrel{\circ}{1}$ & I \\
\hline & 10 & 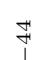 & $\stackrel{20}{=}$ & $\hat{0}$ & $\stackrel{1}{\Rightarrow}$ & $\vec{I}$ & $\check{\infty}$ & ip \\
\hline & r & $\underset{+}{+}$ & 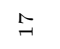 & $\infty$ & $\stackrel{\infty}{\circ}$ & $\infty$ & i & $\curvearrowright$ \\
\hline & $H$ & กิ & 모 & $\begin{array}{c}\infty \\
\infty \\
\infty\end{array}$ & - & 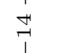 & $\stackrel{\infty}{\sim}$ & 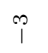 \\
\hline & N & i & $\infty$ & 신 & $\stackrel{8}{\circ}$ & 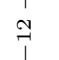 & $\vec{i}$ & 0 \\
\hline & $\rightarrow$ & $\infty$ & $\stackrel{2}{\sim}$ & iิ & 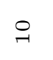 & $\begin{array}{l}1 \\
1 \\
1\end{array}$ & r & $\vec{\imath}$ \\
\hline & o & $\vec{\imath}$ & $\infty$ & & . & $p$ & -1 & 0 \\
\hline & 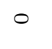 & & & & & ip & 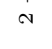 & \\
\hline & : & : & $\stackrel{\circ}{\circ}$ & & & & 0 & \\
\hline
\end{tabular}

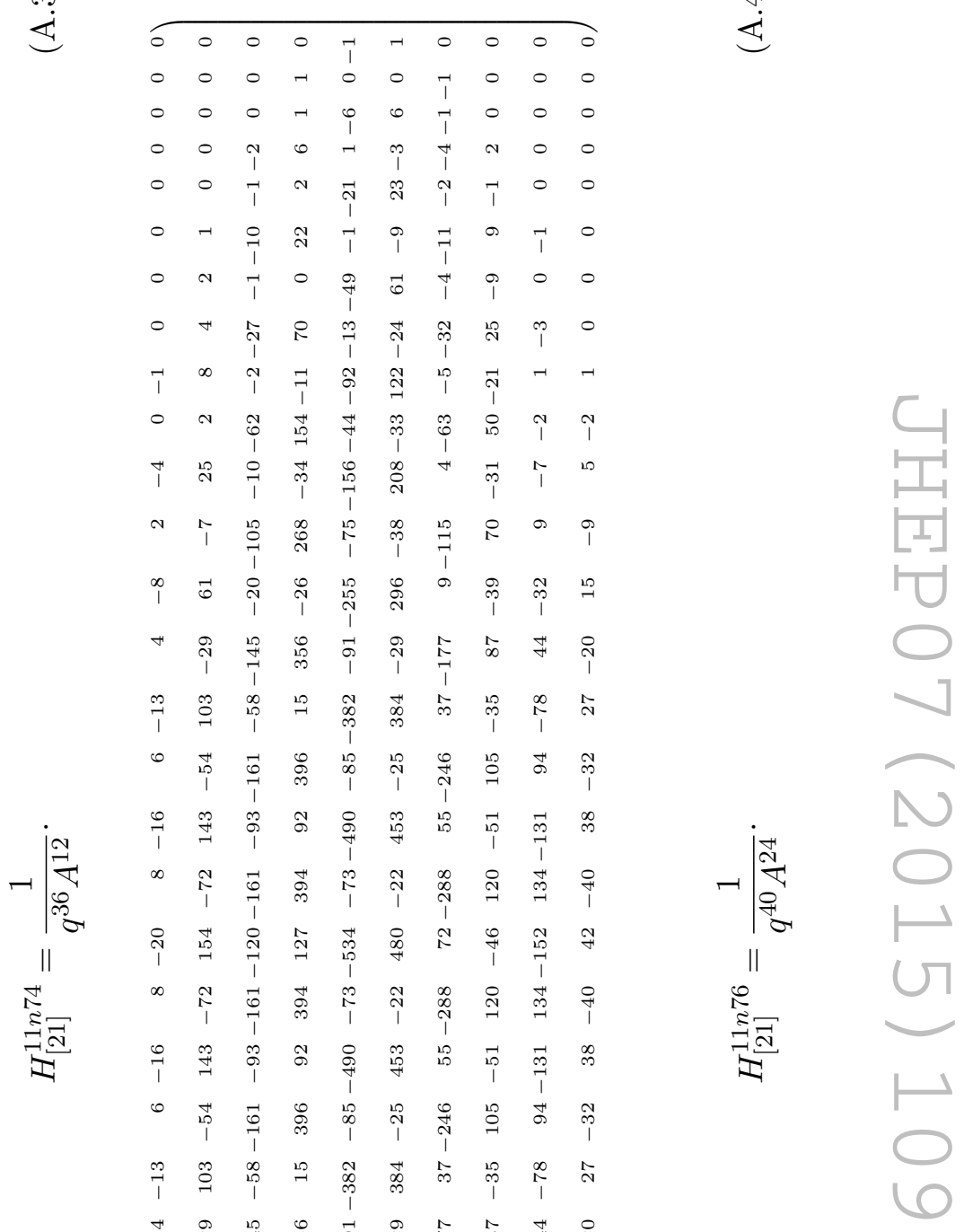

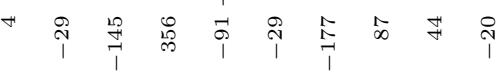

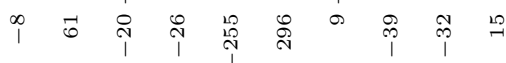

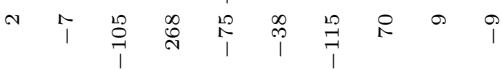

サ

○ N

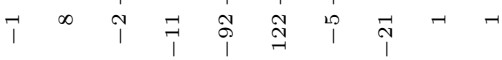

- $\begin{gathered}1 \\ 1\end{gathered}$

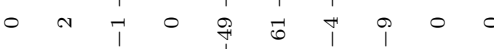

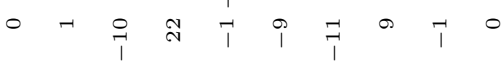

- 0 南a

- 0 i

$\begin{array}{llllllllll}0 & 0 & 0 & - & 0 & 0 & 1 & 0 & 0 & 0 \\ 0 & 0 & 0 & - & 0 & 0 & - & 0 & 0 & 0\end{array}$

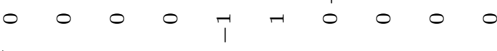




\begin{tabular}{|c|c|c|c|c|c|c|c|c|c|c|c|c|c|c|c|c|c|c|c|c|}
\hline 0 & 0 & $\vec{\imath}$ & $\sim$ & 7 & 0 & 0 & 0 & 0 & a & $\exists$ & $\sigma$ & 0 & -1 & $\sim$ & 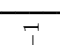 & 0 & 0 & 0 & 0 & 0 \\
\hline 0 & 0 & $d$ & 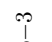 & 0 & $\neg$ & 0 & 0 & 0 & $\circ$ & $\stackrel{+}{*}$ & 0 & 0 & $\alpha$ & $i$ & 1 & - & 0 & 0 & 0 & 0 \\
\hline${ }^{\circ}$ & & $\stackrel{1}{1}$ & $\stackrel{\mathscr{L}}{\sim}$ & $\uparrow$ & 0 & 0 & & 0 & 0 & & 0 & - & $\subsetneq$ & 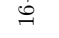 & $\hat{\imath}$ & 0 & 0 & 0 & 0 & 0 \\
\hline 0 & $\vec{\imath}$ & $\stackrel{\infty}{\sim}$ & สิ & $\sim$ & 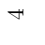 & $\vec{\imath}$ & 0 & 0 & 0 & & 0 & $\vec{\imath}$ & $\stackrel{\infty}{\neg}$ & ฟ & $\sim$ & 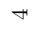 & $\vec{\imath}$ & 0 & 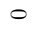 & 0 \\
\hline 0 & 0 & i⿱ & $\mathscr{8}$ & $\vec{N}$ & 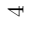 & 0 & 0 & 0 & $\circ$ & & 0 & 0 & i & 8 & $\vec{N}$ & 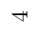 & 0 & 0 & 0 & 0 \\
\hline$\vec{\imath}$ & ${ }_{1}^{\infty}$ & $\mathscr{\infty}$ & IR & ${ }_{1}^{\infty}$ & $\cong$ & $\vec{i}$ & 0 & 0 & 0 & & $\vec{\imath}$ & ${ }_{1}^{\infty}$ & $\mathscr{\infty}$ & Ro & $T$ & $\cong$ & $\vec{i}$ & 0 & 0 & 0 \\
\hline N & $\Re$ & $\stackrel{P}{I}$ & 通 & $\stackrel{\infty}{N}$ & $\exists$ & i & $\rightarrow$ & 0 & 0 & & N & ๙ & $\stackrel{8}{0}$ & $\stackrel{8}{0}$ & ஜి & $\stackrel{7}{7}$ & $i$ & - & 0 & 0 \\
\hline$\hat{\imath}$ & య & 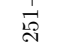 & 苂 & 8 & 18 & $\vec{t}$ & 0 & 0 & 0 & & $\uparrow$ & ఇ్ & $\overrightarrow{\vec{a}}$ & $\stackrel{8}{\stackrel{8}{0}}$ & : & న్ & $\dddot{m}$ & 0 & 0 & 0 \\
\hline \pm & i & $\vec{z}$ & $\frac{1}{\sqrt{~}}$ & ปิ & ๙ & $\cong$ & $\nabla$ & 0 & 0 & & \pm & $\Re$ & $\mathbb{7}$ & $\frac{1}{\vec{~}}$ & $\stackrel{\infty}{\sim}$ & ลิ & $\stackrel{9}{1}$ & $\forall$ & 0 & 0 \\
\hline ণิ & $\vec{\Xi}$ & 点 & 욕 & ஜֶ๊ & $\delta$ & $\stackrel{9}{1}$ & $\sim$ & 0 & 0 & & จิ & $\vec{\Xi}$ & 点 & 気 & 怘 & $\widehat{\infty}^{\infty}$ & $\vec{A}$ & $\sim$ & 0 & 0 \\
\hline F & $\stackrel{\infty}{\infty}$ & $\underset{\substack{10 \\
\infty}}{\infty}$ & $\stackrel{9}{7}$ & $\stackrel{\circledast}{\Im}$ & $\hat{n}$ & Fi & $\exists$ & $\uparrow$ & 0 & & 与 & $\stackrel{\infty}{\infty}$ & 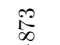 & 子 & $\stackrel{\bigotimes}{\infty}$ & 7 & $\frac{28}{1}$ & $\sigma$ & $\vec{\imath}$ & 0 \\
\hline ֻ & 志 & ज्ञ & $\stackrel{\Omega}{\mathrm{N}}$ & 韩 & R & $\exists$ & $\cong$ & 0 & 0 & & $\stackrel{\infty}{1}$ & 芯 & \& & $\underset{\sim}{\vec{N}}$ & ్ㅗㅇ & 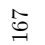 & $\stackrel{0}{1}$ & $\stackrel{2}{9}$ & 0 & 0 \\
\hline$\exists$ & $\underset{\substack{\infty \\
\infty}}{\infty}$ & 品 & 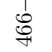 & $\frac{1}{b 2}$ & ڤ్ & $\stackrel{g}{7}$ & $r$ & $\vec{i}$ & 0 & & $\stackrel{\exists}{\exists}$ & $\underset{\infty}{\infty}$ & $\overrightarrow{\mathrm{c}}$ & 字 & $\frac{\infty}{20}$ & F & 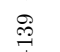 & 10 & 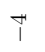 & 0 \\
\hline$\stackrel{\circ}{\leftrightarrow}$ & 通 & 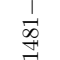 & 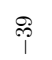 & 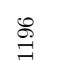 & ॐ & $\stackrel{\circ}{\circ}$ & $\stackrel{8}{9}$ & $\vec{\imath}$ & 0 & & $\stackrel{\mathscr{Q}}{9}$ & 点 & 尚 & ণi i & $\stackrel{\stackrel{g}{g}}{=}$ & 总 & $\mathscr{q}$ & 品 & 0 & 0 \\
\hline$\stackrel{\infty}{\stackrel{一}{⿴ 囗 十 ~}}$ & तु & 范 & 总 & 管 & 号 & $\vec{m}$ & $\stackrel{0}{1}$ & ${ }_{1}^{\infty}$ & 0 & & $\stackrel{\infty}{\stackrel{\sim}{\sim}}$ & : & $\stackrel{\infty}{\infty}$ & న్రి & ळ్ & $\stackrel{\stackrel{\circ}{9}}{\rightarrow}$ & $\vec{\circ}$ & ฐิ & $\stackrel{9}{1}$ & 0 \\
\hline 荢 & $\mathscr{\mathscr { b }}$ & $\stackrel{\substack{\infty \\
\hdashline \\
\hdashline}}{a}$ & 곡 & $\underset{\sim}{\overrightarrow{0}}$ & ฮ్ & $\stackrel{g}{g}$ & $\overrightarrow{\mathrm{I}}$ & i & - & & 荢 & $\vec{g}$ & \begin{tabular}{l}
1 \\
$\substack{\infty \\
\hdashline \\
\hdashline}$
\end{tabular} & 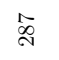 & 量 & 怘 & 呈 & $\overleftrightarrow{\Xi}$ & 0 & - \\
\hline స్ & $\begin{array}{l}\infty \\
\infty \\
\infty \\
\infty\end{array}$ & 葪 & $\stackrel{\circ}{9}$ & 筩 & $\overrightarrow{\mathrm{s}}$ & 崩 & $\underset{\substack{\infty \\
1}}{1}$ & $\vec{\imath}$ & 0 & & 矛 & గ్ర్ & $\overrightarrow{\widehat{N}}$ & $\stackrel{\text { I }}{J}$ & 今ે & g & ㅇํำ & $\begin{array}{l}\infty \\
i \\
1\end{array}$ & $\stackrel{29}{1}$ & 0 \\
\hline 궁 & $\underset{\substack{\infty \\
\infty}}{\infty}$ & 完 & 疍 & 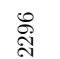 & 옹 & 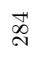 & $\overrightarrow{\mathrm{N}}$ & $\vec{i}$ & $\sim$ & & 府 & $\infty_{\infty}^{\infty}$ & 鴋 & 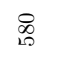 & $\begin{array}{l}\mathscr{D} \\
\text { స్ }\end{array}$ & g & $\stackrel{\text { N }}{N}$ & 太ั & $\sim$ & $\sim$ \\
\hline 范 & $\stackrel{\infty}{\Xi}$ & 䅉 & $\stackrel{\infty}{p}$ & 盆 & $\overrightarrow{⿱ 亠 ⿱ 口 小}$ & o. & 谓 & $\stackrel{20}{1}$ & $\imath$ & & 范 & 范 & 氛 & 号 & $\overrightarrow{\vec{D}}$ & 尔 & 是 & 㫊 & $\vec{i}$ & $\vec{\imath}$ \\
\hline 弈 & $\stackrel{\infty}{\infty}$ & 范 & 员 & 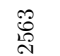 & 喻 & $\vec{\sigma}$ & $\stackrel{\leftrightarrow}{\stackrel{\leftrightarrow}{~}}$ & i & N & $\left.\rightarrow\right|_{0} ^{+}$ & $\stackrel{+}{+}$ & g & 落 & 是 & 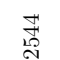 & $\stackrel{\infty}{N}$ & 总 & 品 & - & N \\
\hline 菒 & : & 莺 & $\stackrel{\otimes}{i}$ & 南 & $\stackrel{0}{10}$ & ๙ูণ & $\stackrel{8}{9}$ & $\stackrel{0}{1}$ & 0 & & $\overrightarrow{\mathrm{\sigma}}$ & 茄 & 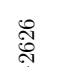 & $\stackrel{\wp}{i}$ & 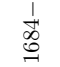 & 嗾 & 象 & 苛 & $\vec{i}$ & o \\
\hline 获 & $\stackrel{\infty}{\infty}$ & 商 & 员 & 旕 & 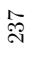 & $\vec{\circ}$ & 今. & $i$ & N & న. & $\overrightarrow{\stackrel{*}{*}}$ & 驾 & 范 & 总 & 䓫 & $\stackrel{\infty}{\text { సे }}$ & 总 & 今. & - & $\sim$ \\
\hline 䒿 & $\stackrel{\infty}{\Xi}$ & 高 & $\stackrel{\infty}{i}$ & 点 & $\sqrt{5}$ & 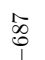 & 岛 & $\stackrel{29}{1}$ & $\grave{\imath}$ & $\mathbb{Z}$ & 萑 & 芯 & 商 & 号 & 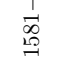 & 䒺 & : & 岂 & $\vec{i}$ & $\vec{\imath}$ \\
\hline 桷 & $\begin{array}{l}\infty \\
\substack{\infty \\
1}\end{array}$ & 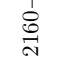 & 㿣 & 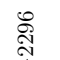 & 옥 & 芯 & $\overrightarrow{\mathrm{N}}$ & $\vec{i}$ & N & & 管 & 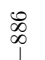 & 煦 & $\underset{\infty}{\infty}$ & $\begin{array}{l}\stackrel{\circ}{0} \\
\text { స్ }\end{array}$ & $\stackrel{8}{\circ}$ & $\stackrel{\text { N. }}{N}$ & สั & N & $\sim$ \\
\hline స్ & 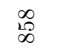 & 岗 & $\stackrel{\circ}{9}$ & 旋 & $\overrightarrow{\mathrm{N}}$ & 娣 & $\underset{\substack{\infty \\
1}}{1}$ & $\overrightarrow{7}$ & 0 & & ড్ & ס્ळ & $\overrightarrow{\mathrm{N}}$ & 今̄ & స్ํำ & ه్ & 离 & $\stackrel{\infty}{\infty}$ & $\stackrel{20}{i}$ & 0 \\
\hline 愙 & $\ddot{\mathscr{o}}$ & $\begin{array}{l}\stackrel{\infty}{\infty} \\
\stackrel{\infty}{\infty}\end{array}$ & ్ㅗ & $\underset{\infty}{\vec{\infty}}$ & 今్ & $\stackrel{\mathscr{0}}{\circ}$ & $\overrightarrow{\mathrm{I}}$ & i & $\tau$ & & 荢 & : & 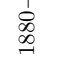 & 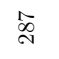 & 茎 & 今 & 㖞 & $\vec{I}$ & $\circ$ & - \\
\hline$\stackrel{\infty}{\stackrel{\infty}{\rightarrow}}$ & ત્త & 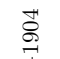 & 鄵 & 点 & 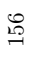 & $\overrightarrow{\vec{d}}$ & $\stackrel{0}{1}$ & ${ }_{i}^{\infty}$ & 0 & & $\stackrel{\infty}{\stackrel{\sim}{\rightarrow}}$ & : & 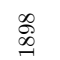 & ๙్లై & 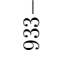 & 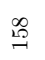 & 藏 & ָิ & $\stackrel{9}{1}$ & 0 \\
\hline 号 & 通 & $\underset{\exists}{\vec{\infty}}$ & $\stackrel{\oplus}{i}$ & $\stackrel{\mathscr{g}}{\exists}$ & $\stackrel{8}{\circ}$ & 号 & 品 & 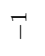 & 0 & & 胃 & 点 & 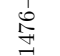 & श्î & $\stackrel{\stackrel{g}{g}}{\leftrightarrows}$ & : & $\mathscr{F}$ & 品 & 0 & 0 \\
\hline$\nRightarrow$ & $\underset{\infty}{\infty}$ & 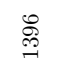 & ஜ & $\frac{1}{b 0}$ & tొ & $\stackrel{P}{7}$ & $r$ & $\vec{i}$ & 0 & & 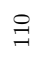 & ळ & 渵 & 号 & $\stackrel{9}{10}$ & F & $\stackrel{\circ}{\rightarrow}$ & 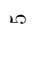 & $\vec{i}$ & 0 \\
\hline$\stackrel{\infty}{\uparrow}$ & 寻 & $\stackrel{1}{\stackrel{I}{\Xi}}$ & $\stackrel{\circ}{\text { ㄱ. }}$ & 늉 & R & 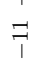 & $\cong$ & 0 & 0 & & $\stackrel{\infty}{\uparrow}$ & 芯 & $\stackrel{1}{\stackrel{1}{8}}$ & $\underset{\sim}{\vec{N}}$ & Fै & : & $\stackrel{\varphi}{1}$ & $\stackrel{9}{2}$ & 0 & 0 \\
\hline F & $\underset{\infty}{\infty}$ & $\underset{\substack{10 \\
\infty}}{ }$ & 孚 & $\stackrel{\wp}{9}$ & 占 & 管 & $\exists$ & $\vec{\imath}$ & 0 & & 궁 & $\underset{\infty}{\infty}$ & 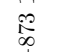 & F & 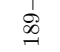 & 7 & 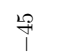 & $\sigma$ & $\vec{\imath}$ & 0 \\
\hline ণิ & $\vec{\Xi}$ & 空 & I্] & :ु & \& & $\stackrel{9}{\overrightarrow{1}}$ & $\sim$ & 0 & 0 & & ণิ & $\vec{s}$ & 总 & 等 & 离 & $\stackrel{\infty}{\infty}$ & $\vec{\top}$ & $\sim$ & 0 & 0 \\
\hline \pm & 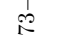 & $\exists$ & $\overrightarrow{\mathrm{\sigma}}$ & N & $\approx$ & $\approx$ & 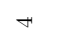 & 0 & 0 & & \pm & 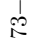 & $\overrightarrow{7}$ & $\frac{1}{\vec{\jmath}}$ & $\stackrel{1}{\sim}$ & ล & $\stackrel{\Im}{I}$ & 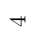 & 0 & 0 \\
\hline$\hat{\imath}$ & $\tilde{\sigma}$ & $\overrightarrow{\underline{s}}$ & 苛 & 8 & 品 & $\vec{t}$ & 0 & 0 & 0 & & $\uparrow$ & مू & $\frac{1}{\vec{n}}$ & 跑 & 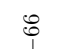 & ळ̆ & $\stackrel{\infty}{I}$ & 0 & 0 & 0 \\
\hline N & $\Re$ & $\stackrel{8}{1}$ & 8 & $\stackrel{\infty}{a}$ & $\exists$ & 广 & $\neg$ & 0 & 0 & & N & ๙ & $\underset{\hdashline}{0}$ & $\stackrel{8}{0}$ & ஜి & $\cong$ & $\uparrow$ & - & 0 & 0 \\
\hline 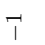 & ${ }_{1}^{\infty}$ & 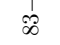 & 橗 & $\phi_{1}^{\infty}$ & $\cong$ & $\vec{i}$ & 0 & 0 & 0 & & $\vec{\imath}$ & $\infty$ & 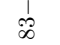 & 20 & $\uparrow$ & $\cong$ & $\ddot{H}$ & 0 & 0 & 0 \\
\hline 0 & 0 & id & ஜg & $\vec{A}$ & $\rightarrow$ & 0 & 0 & 0 & 0 & & 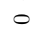 & 0 & iv & 8 & $\vec{\pi}$ & $r$ & 0 & 0 & 0 & 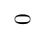 \\
\hline 0 & $\uparrow$ & $\underset{\sim}{\infty}$ & ล & 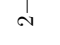 & 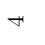 & 7 & 0 & 0 & 0 & & 0 & $\vec{\imath}$ & $\stackrel{1}{\infty}$ & ฟ & 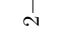 & + & $\vec{\imath}$ & 0 & 0 & 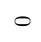 \\
\hline 0 & - & 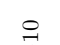 & $\stackrel{b}{b}$ & $\uparrow$ & 0 & 0 & 0 & 0 & 0 & & 0 & - & 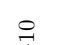 & $\stackrel{\emptyset}{\varrho}$ & $\uparrow$ & 0 & 0 & 0 & 0 & 0 \\
\hline 0 & 0 & 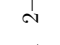 & $i$ & 0 & $\vec{r}$ & 0 & 0 & 0 & 0 & & 0 & 0 & $\stackrel{d}{\alpha}$ & $\uparrow$ & 0 & - & 0 & 0 & 0 & 0 \\
\hline & 0 & $\vec{\imath}$ & $\stackrel{\sim}{\sim}$ & $T$ & 0 & 0 & 0 & 0 & & & c & 0 & $\vec{i}$ & $\alpha$ & $\vec{\uparrow}$ & 0 & 0 & 0 & 0 & \\
\hline
\end{tabular}




\begin{tabular}{|c|c|c|c|c|c|c|c|c|}
\hline & & & & & & & & \\
\hline & $\begin{array}{l}0 \\
0 \\
0\end{array}$ & $\begin{array}{l}0 \\
0 \\
0\end{array}$ & $\begin{array}{l}0 \\
0\end{array}$ & & & & & \\
\hline & $\circ$ & $\circ$ & & & & $\vec{\nexists}$ & 占 & \\
\hline & 0 & 0 & $\vec{\imath}$ & $\frac{1}{\mathrm{I}}$ & ๙ু & 8 & $\vec{m}$ & \\
\hline & 0 & $\vec{\imath}$ & $\stackrel{\Im}{9}$ & 必 & 爵 & 嵒 & 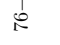 & ф \\
\hline & $\circ$ & p & $\rho_{1}$ & ฐ్ & $\exists$ & 点 & $\stackrel{\mathscr{I}}{1}$ & $\Rightarrow$ \\
\hline & $\circ$ & i & $q$ & 急 & 政 & $\underset{\infty}{\circ}$ & g & $\exists$ \\
\hline & $\sim$ & $\stackrel{\varphi}{1}$ & $\stackrel{\infty}{1}$ & $\frac{1}{\stackrel{s}{g}}$ & $\underset{\exists}{\exists}$ & 䘚 & 胥 & \\
\hline & - & $\bullet$ & 足 & 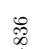 & 点 & 퐇 & 范 & $\vec{\sigma}$ \\
\hline & $\sigma$ & 嗢 & 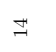 & $\exists$ & 总 & $\begin{array}{c}\infty \\
0 \\
0.0\end{array}$ & $\aleph_{\infty}^{\infty}$ & $\mathbb{E}$ \\
\hline$\vec{i}$ & $\circ$ & 品 & s. & ఇ & 品 & $\infty$ & 8 & \\
\hline & 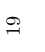 & $\stackrel{\infty}{\exists}$ & s. & 离 & $\stackrel{\infty}{\infty}$ & 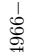 & P & \\
\hline$\vec{i}$ & $\vec{\imath}$ & 8 & s. & 是 & 器 & 㱍 & 递 & \\
\hline $\mathrm{N}$ & $\infty$ & 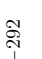 & จิ & 竎 & 点 & 须 & 品 & \\
\hline$\rho_{\uparrow}$ & $\neg$ & : & \& & $\vec{J}$ & $\mathbb{W}_{\mathbb{N}}^{\mathbb{N}}$ & $\begin{array}{l}\infty \\
\infty \\
\infty \\
\infty\end{array}$ & 等 & 잏 \\
\hline+4 & 占 & 胥 & 品 & 颃 & 㖞 & 憙 & $\begin{array}{l}\infty \\
\text { s. }\end{array}$ & \\
\hline 9 & ঙ & ฆิ & $\vec{g}$ & 岕 & ठ & $\begin{array}{r}\vec{\infty} \\
\stackrel{\infty}{\infty}\end{array}$ & 总 & 总 \\
\hline 6 & $\mathrm{E}$ & : & 学 & 急 & $\begin{array}{l}\mathscr{\infty} \\
\stackrel{8}{\circ}\end{array}$ & 导 & 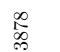 & 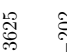 \\
\hline 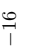 & $\infty$ & $\stackrel{8}{\not}$ & $\vec{\Xi}$ & 总 & 总 & त्ञ & 㗊 & 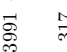 \\
\hline$\infty$ & 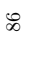 & ఫ్ & 等 & 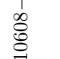 & 㪿 & : & 㽟 & \\
\hline î & i & 字 & $\stackrel{\vec{\Phi}}{\rightarrow}$ & 志 & 旾 & $\begin{array}{l}\infty \\
\stackrel{d}{\vec{d}}\end{array}$ & 윰 & 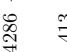 \\
\hline$\infty$ & ஜ & ซ్ & 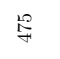 & 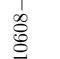 & 莡 & : & 总 & \\
\hline 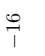 & $\infty$ & $\stackrel{9}{\Rightarrow}$ & $\vec{\Xi}$ & 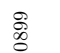 & 党 & त्ञ & 㗊 & 葋 \\
\hline 6 & $\mathrm{E}$ & 䔡 & $\stackrel{\mathscr{P}}{\stackrel{9}{q}}$ & 㤐 & 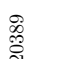 & 方 & 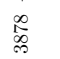 & 能 \\
\hline$\stackrel{m}{i}$ & ঙ & ळ్ & $\overrightarrow{\vec{g}}$ & סूర్ & ठั) & $\begin{array}{l}\vec{\infty} \\
\stackrel{\infty}{o}\end{array}$ & 怘 & 总 \\
\hline+ & 占 & 里 & 융 & 茝 & 骂 & 商 & 怘 & 䢔 \\
\hline$\infty$ & - & 哭 & 8 & $\overrightarrow{\mathbb{O}}$ & 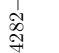 & $\begin{array}{c}\infty \\
\substack{\infty \\
\infty}\end{array}$ & 等 & \\
\hline$\gamma$ & $\infty$ & ฐั & จิ & 童 & 畜 & 节 & 量 & 㝶 \\
\hline it & $\vec{\imath}$ & 8 & 今. & 员 & 兽 & 霝 & 通 & $\bar{\sigma}$ \\
\hline 0 & $\stackrel{9}{9}$ & $\stackrel{\infty}{\exists}$ & $\stackrel{s}{\circ}$ & $\tilde{N}$ & $\stackrel{\varphi}{\infty}$ & . & of & 영 \\
\hline 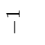 & 0 & 18 & s. & ֻั & 虽 & 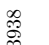 & 洞 & 盙 \\
\hline o & $\sigma$ & 胃 & $\Xi$ & $\frac{1}{\exists}$ & 罯 & $\begin{array}{l}1 \\
\substack{1 \\
0 \\
0}\end{array}$ & 吕 & $\vec{E}$ \\
\hline o & - & 0 & R & 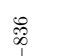 & 赵 & ్ㅠㅁ & 薄 & \\
\hline o & $\sim$ & $\stackrel{\varphi}{\uparrow}$ & $\stackrel{m}{\rightarrow}$ & 占 & $\stackrel{\cong}{\exists}$ & 贞 & 壳 & $\frac{9}{i}$ \\
\hline 0 & 0 & $\uparrow$ & $q$ & 舜 & 变 & $\stackrel{\rho}{\infty}$ & g & $\exists$ \\
\hline o & 0 & i & $\varphi_{1}^{\infty}$ & ฐ్ & $\exists$ & 㤐 & ֻ & $\Rightarrow$ \\
\hline 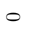 & $\circ$ & $\vec{\imath}$ & $\stackrel{\sim}{\sim}$ & $\stackrel{\infty}{1}$ & 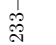 & 㤐 & 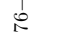 & ${ }_{i}$ \\
\hline$p$ & 0 & 0 & T & $\vec{N}$ & \% & $\frac{1}{\delta}$ & $\overrightarrow{\sigma_{p}}$ & \\
\hline & 0 & 0 & ^ & $\stackrel{\infty}{1}$ & ळे & F & $\stackrel{2}{7}$ & \\
\hline & : & : & $\begin{array}{l}0 \\
0\end{array}$ & $\stackrel{N}{7}$ & i & $\cdots$ & $I$ & \\
\hline
\end{tabular}
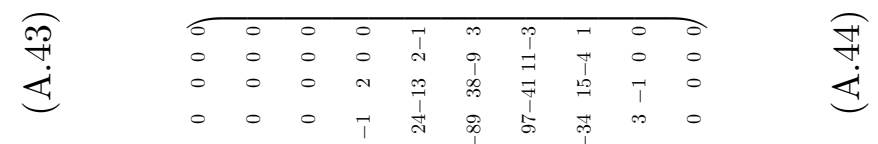

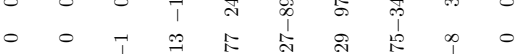

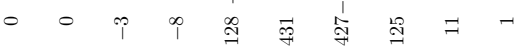

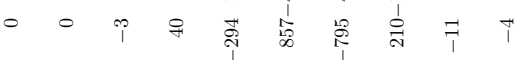

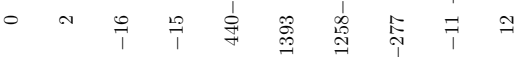

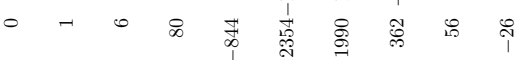

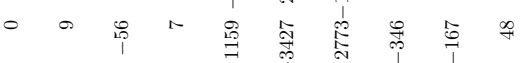

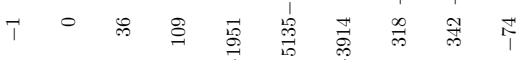

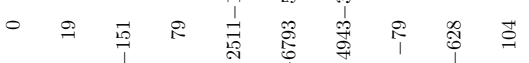

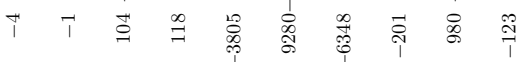

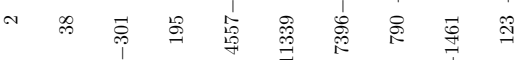

ৎ-

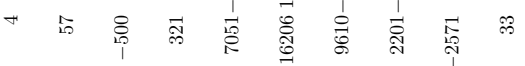

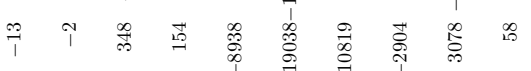

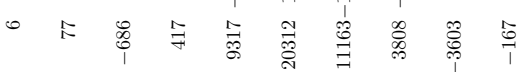

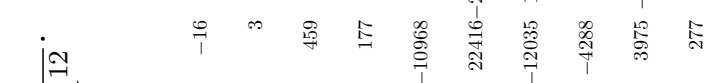

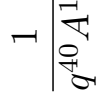

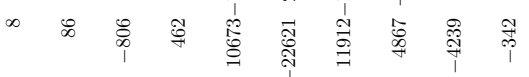

Tิ

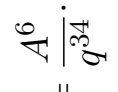

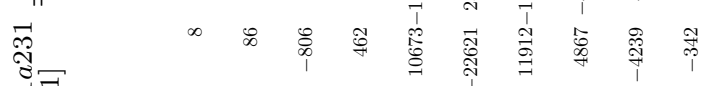

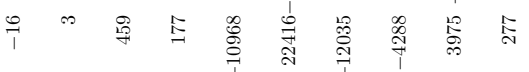

$\sqrt{2}$

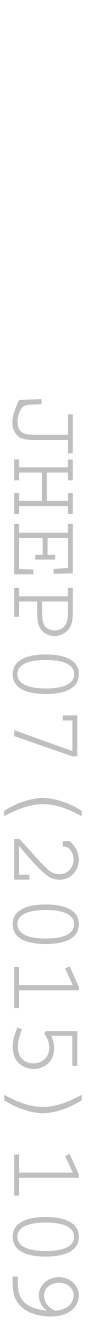

i ๆ

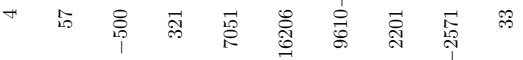

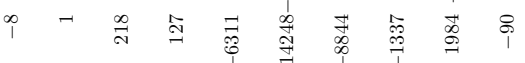

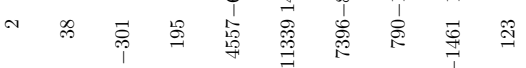

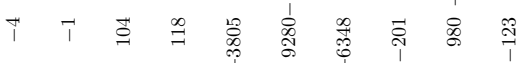

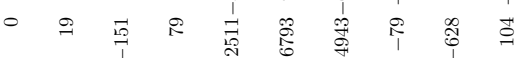

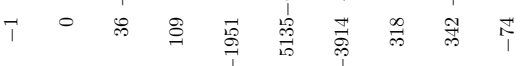

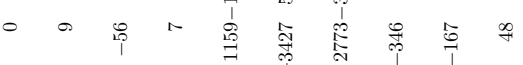

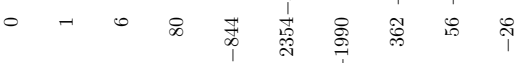

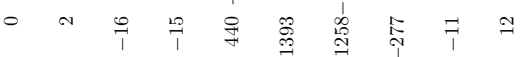

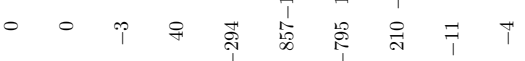

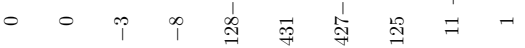

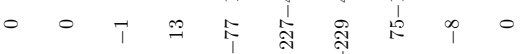

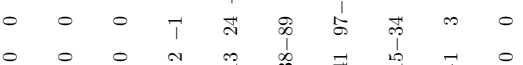

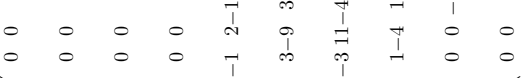



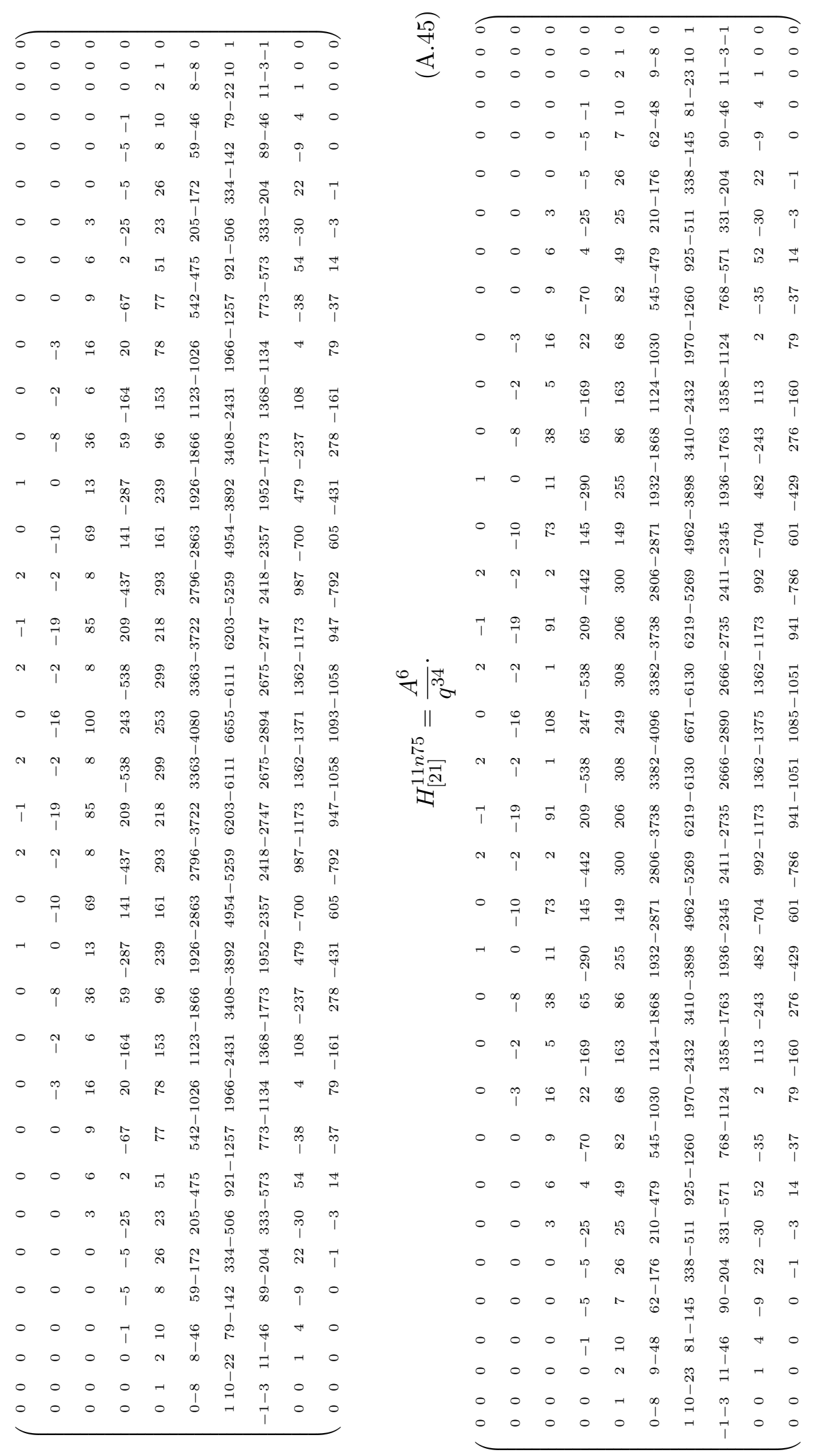
Open Access. This article is distributed under the terms of the Creative Commons Attribution License (CC-BY 4.0), which permits any use, distribution and reproduction in any medium, provided the original author(s) and source are credited.

\section{References}

[1] J.W. Alexander, Topological invariants of knots and links, Trans. Amer. Math. Soc. 30 (1928) 275.

[2] J.H. Conway, Algebraic properties, in Computational problems in abstract algebra, J. Leech ed., Proc. Conf. Oxford 1967, Pergamon Press, Oxford U.K. and New York U.S.A. (1970), pg. 329.

[3] V.F.R. Jones, Index for subfactors, Invent. Math. 72 (1983) 1.

[4] V.F.R. Jones, A polynomial invariant for knots via von Neumann algebras, Bull. AMS 12 (1985) 103.

[5] V.F.R. Jones, Hecke algebra representations of braid groups and link polynomials, Annals Math. 126 (1987) 335 [inSPIRE].

[6] L. Kauffman, State models and the Jones polynomial, Topology 26 (1987) 395.

[7] P. Freyd et al., A new polynomial invariant of knots and links, Bull. AMS 12 (1985) 239.

[8] J.H. Przytycki and K.P. Traczyk, Invariants of links of Conway type, Kobe J. Math. 4 (1987) 115.

[9] S.-S. Chern and J. Simons, Characteristic forms and geometric invariants, Annals Math. 99 (1974) 48 [INSPIRE].

[10] E. Witten, Quantum field theory and the Jones polynomial, Commun. Math. Phys. 121 (1989) 351 [INSPIRE].

[11] N.M. Dunfield, S. Gukov and J. Rasmussen, The superpolynomial for knot homologies, math/0505662 [INSPIRE].

[12] M. Aganagic and S. Shakirov, Knot homology and refined Chern-Simons index, Commun. Math. Phys. 333 (2015) 187 [arXiv:1105.5117] [InSPIRE].

[13] P. Dunin-Barkowski, A. Mironov, A. Morozov, A. Sleptsov and A. Smirnov, Superpolynomials for toric knots from evolution induced by cut-and-join operators, JHEP 03 (2013) 021 [arXiv:1106.4305] [INSPIRE].

[14] I. Cherednik, Jones polynomials of torus knots via DAHA, arXiv:1111.6195 [INSPIRE].

[15] S. Gukov and M. Stošić, Homological algebra of knots and BPS states, Proc. Symp. Pure Math. 85 (2012) 125 [arXiv:1112.0030] [INSPIRE].

[16] S. Nawata, P. Ramadevi, Zodinmawia and X. Sun, Super-A-polynomials for twist knots, JHEP 11 (2012) 157 [arXiv:1209.1409] [InSPIRE].

[17] S. Nawata, P. Ramadevi and Zodinmawia, Colored Kauffman homology and super-A-polynomials, JHEP 01 (2014) 126 [arXiv:1310.2240] [INSPIRE].

[18] I.Cherednik and I.Danilenko, DAHA and iterated torus knots, arXiv:1408.4348.

[19] M. Khovanov, A categorification of the Jones polynomial, Duke Math. J. 101 (2000) 359 [math/9908171] [INSPIRE]. 
[20] M. Khovanov, Patterns in knot cohomology I, Experiment. Math. 12 (2003) 365374 [math/0201306].

[21] M. Khovanov, Categorifications of the colored Jones polynomial, J. Knot Theor. Ramificat. 14 (2005) 111 [math/0302060].

[22] M. Khovanov, sl(3) link homology, Algebr. Geom. Topol. 4 (2004) 1045 [math/0304375].

[23] M. Khovanov, Triply-graded link homology and Hochschild homology of Soergel bimodules, Int. J. Math. 18 (2007) 869885 [math/0510265].

[24] M. Khovanov, Link homology and categorification, math/0605339.

[25] M. Khovanov, Categorifications from planar diagrammatics, arXiv:1008.5084.

[26] M. Khovanov and L. Rozhansky, Matrix factorizations and link homology, Fund. Math. 199 (2008) 1 [math/0401268].

[27] M. Khovanov and L. Rozhansky, Matrix factorizations and link homology II, Geom. Topol. 12 (2008) 1387 [math/0505056].

[28] D. Bar-Natan, On Khovanov's categorification of the Jones polynomial, Alg. Geom. Topol. 2 (2002) 337 [math/0201043].

[29] D. Bar-Natan, Khovanov's homology for tangles and cobordisms, Geom. Topol. 9 (2005) 1443 [math/0410495].

[30] D. Bar-Natan, Fast Khovanov homology computations, J. Knot Theor. Ramificat. 16 (2007) 243255 [math/0606318].

[31] N. Carqueville and D. Murfet, Computing Khovanov-Rozansky homology and defect fusion, Topology 14 (2014) 489 [arXiv:1108.1081] [INSPIRE].

[32] V. Dolotin and A. Morozov, Introduction to Khovanov homologies. I. Unreduced Jones superpolynomial, JHEP 01 (2013) 065 [arXiv:1208.4994] [INSPIRE].

[33] V. Dolotin and A. Morozov, Introduction to Khovanov homologies. II. Reduced Jones superpolynomials, J. Phys. Conf. Ser. 411 (2013) 012013 [arXiv: 1209.5109] [InSPIRE].

[34] V. Dolotin and A. Morozov, Introduction to Khovanov homologies. III. A new and simple tensor-algebra construction of Khovanov-Rozansky invariants, Nucl. Phys. B 878 (2014) 12 [arXiv: 1308.5759] [INSPIRE].

[35] A. Anokhina and A. Morozov, Towards R-matrix construction of Khovanov-Rozansky polynomials. I. Primary T-deformation of HOMFLY, JHEP 07 (2014) 063 [arXiv: 1403.8087] [INSPIRE].

[36] A. Morozov, Challenges of $\beta$-deformation, Theor. Math. Phys. 173 (2012) 1417 [Teor. Mat. Fiz. 173 (2012) 104] [arXiv:1201.4595] [INSPIRE].

[37] A. Anokhina, A. Mironov, A. Morozov and A. Morozov, Knot polynomials in the first non-symmetric representation, Nucl. Phys. B 882 (2014) 171 [arXiv:1211.6375] [INSPIRE].

[38] E. Gorsky, S. Gukov and M. Stosic, Quadruply-graded colored homology of knots, arXiv: 1304.3481 [INSPIRE].

[39] S. Arthamonov, A. Mironov and A. Morozov, Differential hierarchy and additional grading of knot polynomials, Theor. Math. Phys. 179 (2014) 509 [Teor. Mat. Fiz. 179 (2014) 147] [arXiv:1306.5682] [INSPIRE]. 
[40] A. Anokhina and An. Morozov, Cabling procedure for the colored HOMFLY polynomials, Theor. Math. Phys. 178 (2014) 1 [Teor. Mat. Fiz. 178 (2014) 3] [arXiv:1307.2216] [INSPIRE].

[41] J. Gu and H. Jockers, A note on colored HOMFLY polynomials for hyperbolic knots from WZW models, Commun. Math. Phys. 338 (2015) 393 [arXiv:1407.5643] [inSPIRE].

[42] P. Ramadevi, T.R. Govindarajan and R.K. Kaul, Chirality of knots $9_{42}$ and $10_{71}$ and Chern-Simons theory, Mod. Phys. Lett. A 9 (1994) 3205 [hep-th/9401095] [inSPIRE].

[43] S. Nawata, P. Ramadevi and Zodinmawia, Colored HOMFLY polynomials from Chern-Simons theory, J. Knot Theor. Ramificat. 22 (2013) 1350078 [arXiv:1302.5144] [INSPIRE].

[44] Zodinmawia, Knot polynomials from $\mathrm{SU}(N)$ Chern-Simons theory, superpolynomials and super-A-polynomials, Ph.D. thesis, IIT, Mumbai India (2014).

[45] D. Galakhov, D. Melnikov, A. Mironov, A. Morozov and A. Sleptsov, Colored knot polynomials for arbitrary pretzel knots and links, Phys. Lett. B 743 (2015) 71 [arXiv: 1412.2616] [INSPIRE].

[46] A. Mironov, A. Morozov and A. Sleptsov, Colored HOMFLY polynomials for the pretzel knots and links, arXiv:1412.8432 [INSPIRE].

[47] D. Galakhov, D. Melnikov, A. Mironov and A. Morozov, Knot invariants from Virasoro related representation and pretzel knots, arXiv:1502.02621 [INSPIRE].

[48] D. Bar-Natan, The Knot atlas webpage, http://www.katlas.org.

[49] A. Kawauchi, Survey on knot theory, Springer, Germany (1996).

[50] R.K. Kaul and T.R. Govindarajan, Three-dimensional Chern-Simons theory as a theory of knots and links, Nucl. Phys. B 380 (1992) 293 [hep-th/9111063] [InSPIRE].

[51] R.K. Kaul and T.R. Govindarajan, Three-dimensional Chern-Simons theory as a theory of knots and links. 2. Multicolored links, Nucl. Phys. B 393 (1993) 392 [INSPIRE].

[52] P. Rama Devi, T.R. Govindarajan and R.K. Kaul, Three-dimensional Chern-Simons theory as a theory of knots and links. 3. Compact semisimple group, Nucl. Phys. B 402 (1993) 548 [hep-th/9212110] [INSPIRE].

[53] P. Ramadevi, T.R. Govindarajan and R.K. Kaul, Knot invariants from rational conformal field theories, Nucl. Phys. B 422 (1994) 291 [hep-th/9312215] [INSPIRE].

[54] P. Ramadevi, T.R. Govindarajan and R.K. Kaul, Representations of composite braids and invariants for mutant knots and links in Chern-Simons field theories, Mod. Phys. Lett. A 10 (1995) 1635 [hep-th/9412084] [INSPIRE].

[55] P. Ramadevi and T. Sarkar, On link invariants and topological string amplitudes, Nucl. Phys. B 600 (2001) 487 [hep-th/0009188] [INSPIRE].

[56] Zodinmawia and P. Ramadevi, $\mathrm{SU}(N)$ quantum Racah coefficients \& non-torus links, Nucl. Phys. B 870 (2013) 205 [arXiv: 1107.3918] [INSPIRE].

[57] Zodinmawia and P. Ramadevi, Reformulated invariants for non-torus knots and links, arXiv:1209.1346 [INSPIRE].

[58] N. Yu. Reshetikhin and V.G. Turaev, Ribbon graphs and their invariants derived from quantum groups, Commun. Math. Phys. 127 (1990) 1 [INSPIRE]. 
[59] E. Guadagnini, M. Martellini and M. Mintchev, Chern-Simons field theory and quantum groups, in Proceedings of the Workshop on Quantum Groups, Clausthal 1989, World Scientific, Singapore (1989), pg. 307 [Lect. Notes Phys. 370 (1990) 307] [InSPIRE].

[60] E. Guadagnini, M. Martellini and M. Mintchev, Chern-Simons holonomies and the appearance of quantum groups, Phys. Lett. B 235 (1990) 275 [INSPIRE].

[61] V.G. Turaev and O.Y. Viro, State sum invariants of 3 manifolds and quantum $6 j$ symbols, Topology 31 (1992) 865 [INSPIRE].

[62] A. Morozov and A. Smirnov, Chern-Simons theory in the temporal gauge and knot invariants through the universal quantum R-matrix, Nucl. Phys. B 835 (2010) 284 [arXiv:1001.2003] [INSPIRE].

[63] A. Smirnov, Notes on Chern-Simons theory in the temporal gauge, in Proc. of International School of Subnuclear Phys., Erice Italy (2009) [Subnucl. Ser. 47 (2011) 489] [arXiv:0910.5011] [INSPIRE].

[64] A. Mironov, A. Morozov and An. Morozov, Character expansion for HOMFLY polynomials. II. Fundamental representation. Up to five strands in braid, JHEP 03 (2012) 034 [arXiv:1112.2654] [INSPIRE].

[65] A. Mironov, A. Morozov and A. Morozov, Character expansion for HOMFLY polynomials. I. Integrability and difference equations, in Strings, Gauge Fields, and the Geometry Behind: The Legacy of Maximilian Kreuzer, A. Rebhan, L. Katzarkov, J. Knapp, R. Rashkov and E. Scheidegger eds., World Scientific, Singapore (2013), pg. 101 [arXiv:1112.5754].

[66] H. Itoyama, A. Mironov, A. Morozov and An. Morozov, Character expansion for HOMFLY polynomials. III. All 3-strand braids in the first symmetric representation, Int. J. Mod. Phys. A 27 (2012) 1250099 [arXiv:1204.4785] [INSPIRE].

[67] A. Anokhina, A. Mironov, A. Morozov and An. Morozov, Racah coefficients and extended HOMFLY polynomials for all 5-, 6- and 7-strand braids, Nucl. Phys. B 868 (2013) 271 [arXiv:1207.0279] [INSPIRE].

[68] H. Itoyama, A. Mironov, A. Morozov and A. Morozov, Eigenvalue hypothesis for Racah matrices and HOMFLY polynomials for 3-strand knots in any symmetric and antisymmetric representations, Int. J. Mod. Phys. A 28 (2013) 1340009 [arXiv:1209.6304] [inSPIRE].

[69] A. Anokhina, A. Mironov, A. Morozov and An. Morozov, Colored HOMFLY polynomials as multiple sums over paths or standard Young tableaux, Adv. High Energy Phys. 2013 (2013) 931830 [arXiv: 1304.1486] [INSPIRE].

[70] A. Mironov, A. Morozov and A. Morozov, Evolution method and "differential hierarchy" of colored knot polynomials, AIP Conf. Proc. 1562 (2013) 123 [arXiv:1306.3197] [InSPIRE].

[71] A. Mironov, A. Morozov and S. Natanzon, Integrability properties of Hurwitz partition functions. II. Multiplication of cut-and-join operators and WDVV equations, JHEP 11 (2011) 097 [arXiv: 1108.0885] [inSPIRE].

[72] A. Mironov, A. Morozov and S. Natanzon, Complete set of cut-and-join operators in Hurwitz-Kontsevich theory, Theor. Math. Phys. 166 (2011) 1 [arXiv:0904.4227] [InSPIRE].

[73] A. Mironov, A. Morozov and S. Natanzon, Algebra of differential operators associated with Young diagrams, J. Geom. Phys. 62 (2012) 148 [arXiv: 1012.0433] [InSPIRE].

[74] H. Morton and P. Cromwell, Distinguishing mutants by knot polynomials, J. Knot Theor. Ramificat. 5 (1996) 225. 
[75] J. Murakami, Finite type invariants detecting the mutant knots, in Knot Theory, a volume dedicated to Professor Kunio Murasugi for his $70^{\text {th }}$ birthday, M. Sakuma et al. eds., Osaka University, Osaka Japan (2000), pg. 258.

[76] M. Rosso and V.F.R. Jones, On the invariants of torus knots derived from quantum groups, J. Knot Theor. Ramificat. 2 (1993) 97.

[77] X.-S. Lin and H. Zheng, On the Hecke algebras and the colored HOMFLY polynomial, Trans. Amer. Math. Soc. 362 (2010) 1 [math/0601267].

[78] A. Mironov, A. Morozov and An. Morozov, On colored HOMFLY polynomials for twist knots, Mod. Phys. Lett. A 29 (2014) 1450183 [arXiv:1408.3076] [INSPIRE].

[79] S. Nawata, P. Ramadevi and Zodinmawia, Multiplicity-free quantum $6 j$-symbols for $U_{q}\left(s l_{N}\right)$, Lett. Math. Phys. 103 (2013) 1389 [arXiv:1302.5143] [INSPIRE].

[80] H. Itoyama, A. Mironov, A. Morozov and An. Morozov, HOMFLY and superpolynomials for figure eight knot in all symmetric and antisymmetric representations, JHEP 07 (2012) 131 [arXiv: 1203.5978] [INSPIRE].

[81] S. Zhu, Colored HOMFLY polynomials via skein theory, JHEP 10 (2013) 229 [arXiv: 1206.5886] [INSPIRE].

[82] A. Morozov, Special colored superpolynomials and their representation-dependence, JHEP 12 (2012) 116 [arXiv:1208.3544] [INSPIRE].

[83] A. Morozov, The first-order deviation of superpolynomial in an arbitrary representation from the special polynomial, JETP Lett. 97 (2013) 171 [arXiv:1211.4596] [INSPIRE].

[84] A. Mironov, A. Morozov and A. Sleptsov, Genus expansion of HOMFLY polynomials, Theor. Math. Phys. 177 (2013) 1435 [Teor. Mat. Fiz. 177 (2013) 179] [arXiv:1303.1015] [InSPIRE].

[85] A. Mironov, A. Morozov and A. Sleptsov, On genus expansion of knot polynomials and hidden structure of Hurwitz tau-functions, Eur. Phys. J. C 73 (2013) 2492 [arXiv: 1304.7499] [INSPIRE].

[86] A. Mironov, A. Morozov, A. Sleptsov and A. Smirnov, On genus expansion of superpolynomials, Nucl. Phys. B 889 (2014) 757 [arXiv:1310.7622] [INSPIRE].

[87] A. Stoimenow, Tabulating and distinguishing mutants, Int. J. Alg. Comput. 20 (2010) 525.

[88] A. Stoimenow, Knot data tables webpage, http://stoimenov.net/stoimeno/homepage/ptab/index.html.

[89] D. De Wit and J. Links, Where the Links-Gould invariant first fails to distinguish nonmutant prime knots, math/0501224.

[90] S. Nawata, P. Ramadevi and V.K. Singh, Colored HOMFLY polynomials can distinguish mutant knots, arXiv:1504.00364 [INSPIRE]. 